
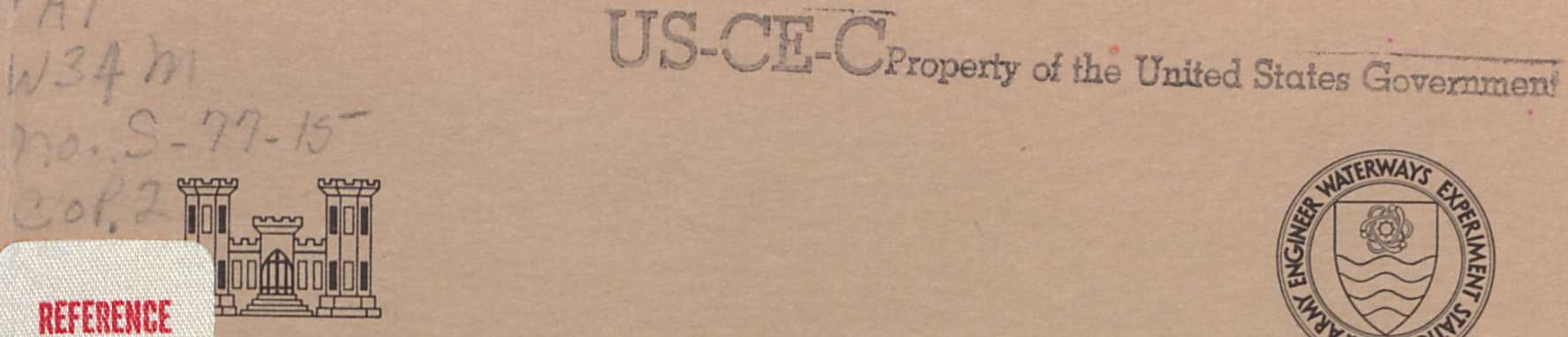

MISCELLANEOUS PAPER S-77-15

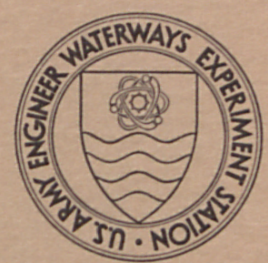

\title{
MATERIALS EVALUATED AS POTENTIAL SOIL STABILIZERS
}

by

Jessie C. Oldham, Royce C. Eaves, Dewey W. White, Jr.

Soils and Pavements Laboratory

U. S. Army Engineer Waterways Experiment Station

P. O. Box 631, Vicksburg, Miss. 39180

September 1977

Final Report

Approved For Public Release; Distribution Unlimited

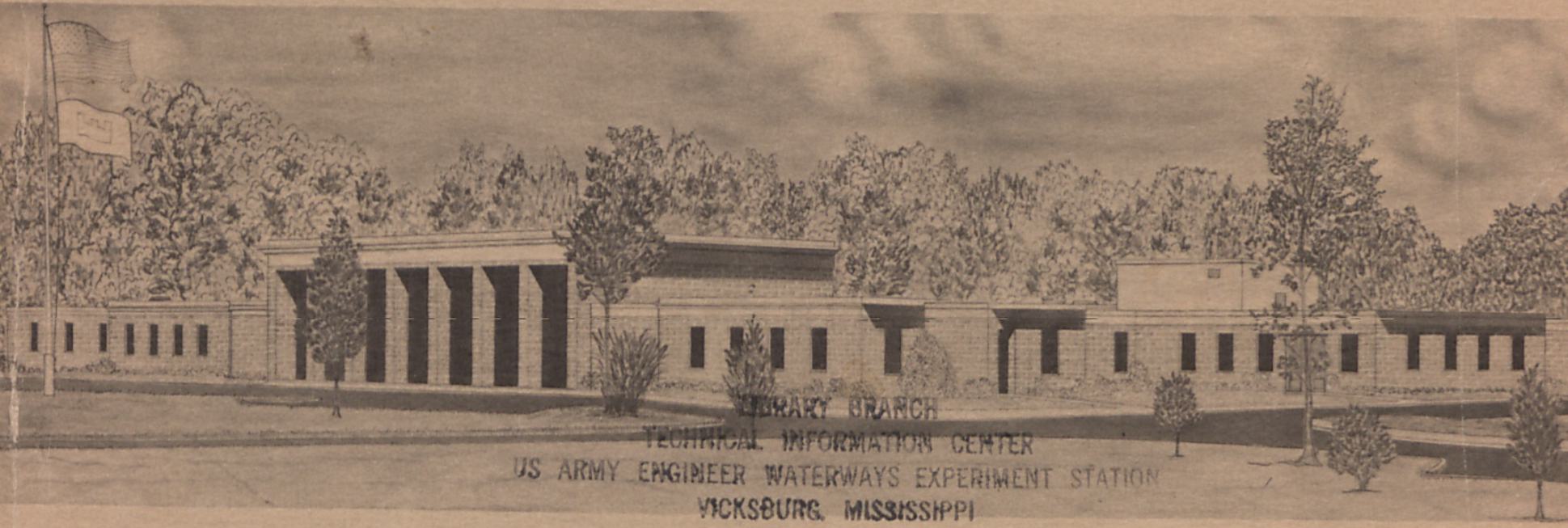

Prepared for Office, Chief of Engineers, U. S. Army

Washington, D. C. $203 / 4$

and

U. S. Army Materiel Development \& Readiness Command

5001 Eisenhower Avenue

Alexandria, Va. 22333

Under Projects 4A7627I9AT40 and ITI6211A528 
Unclassified

SECURITY CLASSIFICATION OF THIS PAGE (Whon Date Entered)

\begin{tabular}{|c|c|}
\hline REPORT DOCUMENTATION PAGE & $\begin{array}{l}\text { READ INSTRUCTIONS } \\
\text { BEFORE COMPLETING FORM }\end{array}$ \\
\hline $\begin{array}{l}\text { 1. REPORT NUMBER } \\
\text { Miscellaneous Paper S-77-15 }\end{array}$ & 3. RECIPIENT'S CATALOG NUMBER \\
\hline $\begin{array}{l}\text { M. TITLE (end Subttio) } \\
\text { MATERIALS EVALUATED AS POTENTIAL SOIL STABILIZERS }\end{array}$ & $\begin{array}{l}\text { 5. TYPE OF REPORT \& PERIOD COVERED } \\
\text { Fingl report }\end{array}$ \\
\hline & 6. PERFORMING ORG. REPORT NUMBER \\
\hline $\begin{array}{l}\text { 7. AUTHOR(a) } \\
\text { Jessie C. Oldham, Royce C. Eaves, } \\
\text { Dewey W. White, Jr. }\end{array}$ & 8. CONTRACT OR GRANT NUMBER(O) \\
\hline $\begin{array}{l}\text { 9. PERFORMING ORGANIZATION NAME AND ADDRESS } \\
\text { U. S. ArmY Engineer Waterways Experiment Station } \\
\text { Soils and Pavements Laboratory } \\
\text { P. O. Box 63l, Vicksburg, Miss. } 39180\end{array}$ & $\begin{array}{l}\text { 10. PROGRAM ELEMENT. PROJECT, TASK } \\
\text { AREA \& WORK UNIT NUMBERS } \\
\text { OCE - Project 4A762719AT40 } \\
\text { DARCOM - Project ITI6211A528 }\end{array}$ \\
\hline $\begin{array}{l}\text { 11. CONTROLLING OFFICE NAME AND ADDRESS } \\
\text { Office, Chief of Engineers, U. S. Army, Washington, }\end{array}$ & $\begin{array}{l}\text { 12. REPORT DATE } \\
\text { September } 1977\end{array}$ \\
\hline $\begin{array}{l}\text { D. C. } 20314 \text {; and U. S. Army Materiel Development } \\
\text { and Readiness Command, Alexandria, Va. } 22333\end{array}$ & $\begin{array}{l}\text { 13. NUMBER OF PAGES } \\
274\end{array}$ \\
\hline \multirow[t]{2}{*}{ 74. MONITORING AGENCY NAME ADDRESS(II dillorent froon Controlline Office) } & $\begin{array}{l}\text { 15. SECURITY CLASS. (of thle roport) } \\
\text { Unclassified }\end{array}$ \\
\hline & 15. DECLASSIFICATION/DOWNGRADING \\
\hline
\end{tabular}

\section{DISTRIBUTION STATEMENT (Of thie Roport)}

Approved for public release; distribution unlimited.

17. DISTRIBUTION STATEMENT (of the abotract entored in Block 20, If difforent tron Roport)

18. SUPPLEMENTARY NOTES

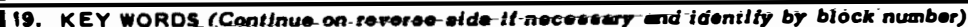

Chemical soll stabilization

Stabilizers (Agents)

Soll stabilization

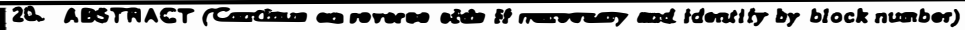

This report documents the history of a program initiated in 1946 to evaluate materials for use as chemical soil stabilizers by the military. A number of Government agencies, universities, and private firms were involved in the program. In addition, many other companies submitted candidate materials for evaluation. Appendix A presents documentation of all materials evaluated including category, components, cost, applicability with regard to soil type, mixing capability, and related effectiveness. Also given are (Continued) 


\section{ABSTRACT (Continued)}

the agencies which conducted the evaluation and appropriate references in which more detailed information of the materials can be found.

The most effective soil stabilizers and the compressive strengths produced by them in four general types of soils are summarized as follows:

Unconfined Compressive Strengths, psi

\begin{tabular}{|c|c|c|c|c|c|}
\hline Soil Type & Untreated & Cement & Lime & Asphalt & Other Best Material \\
\hline Silt & 20 & $80-280$ & $230-860$ & 225 & Sodium silicate - 650 \\
\hline Loess & 20 & 100 & $160-970$ & - & Powders $A$ and $B-385$ \\
\hline Clay & 20 & $76-300$ & $100-340$ & $104-289$ & Calcium oxide - 315 \\
\hline Sand & 20 & $150-425$ & - & -- & Aropol $7110-1170-189$ \\
\hline
\end{tabular}


THE CONTENTS OF THIS REPORT ARE NOT TO BE USED FOR ADVERTISING, PUBLICATION, OR PROMOTIONAL PURPOSES. CITATION OF TRADE NAMES DOES NOT CONSTITUTE AN OFFICIAL ENDORSEMENT OR APPROVAL OF THE USE OF SUCH COMMERCIAL PRODUCTS. 


\section{PREFACE}

Efforts to find a method of solidifying or stabilizing soils for military operations were initiated in May 1946 by the U. S. Army Corps of Engineers. From its beginning in 1946 to 1975, this program of tests to evaluate potential stabilization materials was conducted under the sponsorship of the Office, Chief of Engineers, U. S. Army, and the U. S. Army Materiel Development and Readiness Command. Various private firms also were involved with the tests as well as the U. S. Army Engineer Research and Development Laboratories (now the U.S. Army Mobility Equipment Research and Development Command) and the U. S. Army Engineer Waterways Experiment Station (WES).

This report was prepared at WES by Messrs. Jessie C. Oldham, Royce C. Eaves, and Dewey W. White, Jr., of the Materiel Development Division (MDD), Soils and Pavements Laboratory (S\&PL), under the direct supervision of Messrs. William L. McInnis, Chief, MDD, and James P. Sale, Chief, S\&PL.

Directors of WES during preparation of this report were COL G. $H$. Hilt, CE and COL J. L. Cannon, CE. Mr. F. R. Brown was Technical Director. 
$\underline{\text { Page }}$

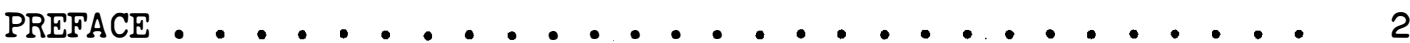

CONVERSION FACTORS, U. S. CUSTOMARY TO METRIC (SI)

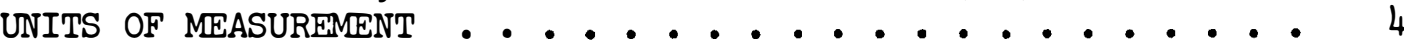

BACKGROUND ......................... 5

PURPOSE . . . . . . . . . . . . . . 5

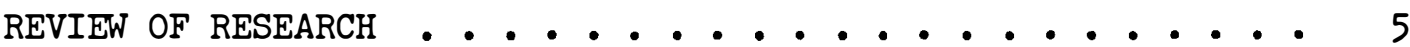

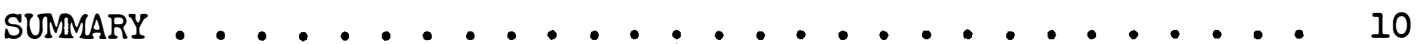

DISCUSSION ......................... 10

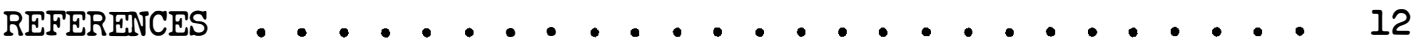

TABLES $1-3$

APPENDIX A: DOCUMENTATION OF MATERIALS EVALUATED . . . . Al

CATEGORY : ACID ........................ A5

CATEGORY： ASPHALT . . . . . . . . . A2I

CATEGORY: CEMENT ................. A650

CATEGORY: LIME. .................... Al30

CATEGORY: RESIN ........................ Al55

CATEGORY: SALT . . . . . . . . Al78

CATEGORY: SILICATE ................... Al81

CATEGORY : OTHER ......................... AI99 
CONVERSION FACTORS, U. S. CUSTOMARY TO MEIRIC (SI)

UNITS OF MEASUREMENT

U. S. customary units of measurement used in this report can be converted to metric (SI) units as follows:

\begin{tabular}{llll}
\multicolumn{1}{c}{ Multiply } & \multicolumn{1}{c}{ By } & & To Obtain \\
\cline { 1 - 2 } inches & 25.4 & millimetres \\
pounds (mass) & 0.4535924 & kilograms \\
pounds (force) & 4.448222 & newtons \\
pounds (force) per square inch & 6.894757 & kilopascals \\
cubic feet & 0.02831685 & cubic metres \\
square yards & 0.8361274 & square metres \\
Fahrenheit degrees & $5 / 9$ & Celsius degrees or Kelvins*
\end{tabular}

* To obtain Celsius (C) temperature readings from Fahrenheit ( $F$ ) readings, use the following formula: $C-(5 / 9)(F-32)$. To obtain Kelvin $(K)$ readings, use: $K=(5 / 9)(F-32)+273.15$. 


\section{Background}

1. In 1946, the U. S. Army Corps of Engineers (CE) initiated a research and development program having the objective of developing improved materials and methods to expeditiously solidify or stabilize soils for use in construction of roads and airfields and in support of military operations over soft ground. During the period 1946-1954, the U. S. Army Engineer Research and Development Laboratories (now the U. S. Army Mobility Equipment Research and Development Command (MERADCOM)) was responsible for the stabilization program. In 1954, the program was assigned to the U. S. Army Engineer Waterways Experiment Station (WES), where research has continued to the present time.

\section{Purpose}

2. This report is being published to document this study of chemical soil stabilization. Through both in-house research and contracted efforts, a wide range of materials was tested and this document serves to record all materials evaluated. This report is not intended to provide guidance in selection of materials or in construction methods. Additional information on each material is provided in the listed reference. Guidance in material selection and construction methods is provided in WES Miscellaneous Paper S-74-23, "Soil Stabilization for Roads and Airfields in the Theater of Operations,"- by. W.- N. Brabston and-G. M. Hammitt, II, September 1974.

\section{Review of Research}

3. From 1946-1955, extensive literature reviews and limited laboratory studies were performed, initially under contract with the Massachusetts Institute of Technology (MIT), the University of California at Los Angeles, and Cornell University. Extensive contract work was 
performed by MIT on stabilizer material development with emphasis on resin systems including melamines, furfurals, formaldehydes, ureas, silicates, acrylamides, vinyls, styrenes, epoxies, and acrylates. Special attention was given to calcium acrylate, which had been found unique in. developing high strength in very wet soils. This polymer resin was studied extensively by MERADCOM both in the laboratory and in the field. Although unique in its mechanism, calcium acrylate had the disadvantages of high cost; the need for large quantities for effective use; heavy dependency on soil type, catalyst, and degree of mixing; and water sensitivity. Much work was devoted to studies of mixing and to development of a field mixing unit to apply calcium acrylate and of various additives to aid incorporation.

4. Simultaneously, Cornell University undertook a contract study of the fundamental properties of clay-water systems and their relation to engineering behavior of soils. Additional work by Cornell was devoted to lignin and chrome-lignin systems for stabilization. The concept of using chrome-lignin to prepare small pillow-shaped briquets of stabilized soil that could be used as artificial aggregate or fill material to bridge weak areas was explored. This technique was tested in the field but was determined to be impractical due to large-scale production requirements and mixing problems in plant production.

5. During this same period, several miscellaneous studies were performed including soil compaction by vibration (California Institute of Technology), low-angle X-ray scattering in soils (Armour Research Foundation), stabilizing soils by freezing (U. S. Bureau of Mines), and theoretical analysis of thin flexible surfaces under load over flexible subgrades (MIT).

6. Following the transfer of responsibility to WES in 1954, a series of state-of-the-art sumary reviews of various soil stabilizing methods and materials was prepared including lignins (1955), calcium acrylate (1956); soil-cement (1956), bituminous materials (1956), lime (1957), mixing principles and equipment (1961), and electrical stabilization (1961). The stabilization problem was defined objectively in terms of specific military road and airfield operational needs, and realistic 
requirements and criteria for various stabilization situations or categories were established to afford direction to the research program. These requirements have been revised periodically to conform to changing military operational concepts and needs.

7. Continued research by MIT from 1955-1961 was directed toward improving the capabilities of conventional stabilizers (asphalts, cement, and lime) and developing new stabilizing systems. This research led to the concept of stabilization with chemicals that attack and react with certain constituents of soil, forming cementitious products in situ. This approach resulted in extensive studies of acid and acid-forming systems, notably the phosphoric acid compounds, and led to improved chemically modified asphalt, cement, and lime systems. Additionally, research by MIT included new resin systems, soil-modifier systems, special asphalt emulsions, and sodium silicate formulations. From 1962-1972, MIT research was directed toward the development of a more fundamental understanding of the structural behavior of stabilized soil and the elucidation of the basic strength-producing mechanisms, both chemical and physical, of soil-additive systems. One phase of research completed by MIT was concerned with the chemical stabilization of selected tropical soils. The results confirmed the utility of cement and lime for improving a spectrum of initially weak soils of tropical origin for military mobility purposes.

8. A contract research effort was conducted by Cornell during 19641968 to establish the feasibility of electrokinetic processes for stabilization of soils for military mobility purposes. The study included. uses of theoretical concepts of electrokinetics, laboratory investigations, and a field test program which involved the unique use of metal mat as one of the electrodes and embedded metal rods as the other electrode. The use of electrical energy to increase soil strength both by dewatering and by electrochemical infection was determined to be feasible, but the benefits achieved were highly dependent upon soil type and conditions, and considerable time was required to achieve significant increases.

9. Contract work was conducted during 1965-1974 by the University of California at Berkeley to investigate the influence of repetitive 
loading on stabilized soil behavior. The results of this work have assisted in establishing appropriate design criteria for stabilized soil layers and procedures for the most efficient use of stabilizing materials.

10. During the time that this project has been assigned to WES, the soil stabilization research and development effort has consisted of two phases, contract research and in-house research. These two phases are closely linked. Contract research has been monitored closely, and materials showing potential have been examined in the in-house research program. In-house efforts have consisted of monitoring technical publications for potential materials or methods and testing and evaluation of materials submitted from industry or discovered in the literature. Positive results obtained from contract research have been explored further in laboratory and field testing. Contract reports documenting these results have been published, and in-house research of significance has been reported and made available to other Government agencies and other interested parties.

11. Materials showing significant potential have been fully evaluated in the laboratory, and field test sections have been constructed at WES and trafficked. Significant and major investigations are listed in the following paragraphs.

a. Calcium acrylate was investigated by MIT and a test lane was constructed at MERADCOM prior to the soil stabilization program being moved to WES. Test lanes were constructed, tested, and evaluated at WES in 1955. Performance of this material was extremely good, but calcium acrylate was later dropped from consideration since it could not withstand rainfall and was too costly.

b. Quicklime was evaluated as a soil stabilizer in laboratory and fileld tests during 1956-1957. Field tests indicated this material could stabilize weak, wet soils very rapidly; however, nonuniform strength resulted because proper mixing was very difficult to obtain.

c. Major research was conducted in 1958 on the use of chemically modified cement in soil stabilization. Laboratory investigations were conducted to determine how various chemicals in combination with portland cement would perform as soil stabilizers. A number of materials were investigated in the laboratory, and results indicated sodium sulfate with cement alone. 
d. Another major research project was conducted in 1958 consisting of laboratory and field investigations of phosphorus pentoxide as a soil stabilization chemical. Results indicated that phosphorus pentoxide had excellent potential for stabilizing some soils; however, traces of calcium carbonate in some soils partially neutralized the effects, and the rapid reaction of this material in wet soils left insufficient time for adequate mixing and compaction.

e. Major research in 1959 was directed toward developing additional information on the use of quicklime as a stabilizer of wet, weak soils for use by the military. Laboratory and field tests indicated the need for additional research to improve quicklime stabilization by chemical modification with supplementary secondary additives to overcome certain limitations.

f. Additional research was conducted in 1960-1961 on the use of supplementary chemicals to enhance the stabilization benefits of quicklime. Laboratory and field tests proved that a number of chemicals were beneficial in lime stabilization; however, the best of these was magnesium sulfate. Laboratory and field tests proved that use of magnesium sulfate in combination with the quicklime resulted in an agent that was much more effective than quicklime alone.

B. During 1955-1961, seven summary reviews were made and the results published concerning soil stabilization processes. These reviews covered work at WES and MERADCOM and literature surveys of work by others. The purposes of these

- reviews were to outline work by the military and others and to document advantages and disadvantages of various stabilizers.

h. Laboratory and field studies were conducted during 1961-1962 on stabilization of soils using portland cement with sodium hydroxide. These investigations indicated that appreciable benefits could be achieved in some soils using sodium hydroxide as a modifier. Excellent tolerance to wetting was achieved using these materials as stabilizing agents.

i. A program was conducted during 1963-1964 to develop design data on cement-stabilized soils. Variables included different strength subgrades, different thicknesses of stabilized layers, varied rates of cement treatment, and four different wheel loadings. A great amount of data was developed and used to verify or generate design criteria for stabilized layers.

1. From 1966-1972, research and development for dust control was conducted. The concept used was to develop a surface stabilizer, a spray-on system versus admix-type stabilization, to achieve strengths. 


\section{Summary}

12. The number of materials and secondary additives tested in this program were many. The materials have been divided into two groups-effective and noneffective. Table 1 contains the materials that had some degree of effectiveness. Table 1 lists the basic materials, secondary materials or additives, soil type, and the number of the page in Appendix $A$ of this report on which detailed information is presented. Table 2 lists materials that had no appreciable effect and did not effect a significant change in the soil parameters. Table 2 is similar to Table 1; however, no individual pages of detailed information are provided. Table 3 lists the best materials for each soil type; effective unconfined compressive strengths are listed.

\section{Discussion}

13. A wide range of materials was evaluated, both in the laboratory and in the field, during the course of this program. Basically, cement, lime, and asphalt were proven to be the better materials for strength stabilization. Research indicated that certain additives used with these materials in trace amounts either increased the strength developed or made the materials effective over a wider range of soils.

14. Also, other materials may be considered for use. These materials are effective in some soils and are economically feasible. Brief statements about these materials are listed below.

a. Lignin or ligno sulphonate is a waste product from paper pulp manufacture. This material is an effective stabilizer and dust control agent for some silt and clay soils. The material is either free or very inexpensive, but laboratory tests should be conducted to determine its effectiveness on soils before large-scale field use is planned.

b. Phosphoric acid and phosphorus pentoxide are effective stabilizers for some clay soils. These materials are hazardous and should be used carefully in the laboratory and the field.

c. Aniline furfural resin is a highly effective waterproofing agent when admixed into clay soils. Permanent waterproofing 
can be achieved with 1 to 3 percent of the resin. Aniline is highly toxic and should be used only after reviewing necessary precautions.

d. A number of materials were investigated for dust control for military purposes. This group of materials is listed in WES Miscellaneous Paper S-69-1, "Materials Investigated for Dust-Control Program (Southeast Asia)," by D. W. White and J. L. Decell, January 1969.

15. The documentation of materials tested is a method of providing guidance for later research. The program has been continued over a period of years. Many organizations and people have been involved. The list includes Government agencies, universities, and private firms. Ideas, concepts, and requirements were changed several times during the duration of the program, and information presented in the tables and in Appendix $A$ is of a general nature. The appropriate referenced reports should be referred to for specific information about materials, test techniques, soils used, and results. 
REFERENCES

1. Armour Research Foundation, "Low-Angle X-Ray Scattering Study on Soils," October 1953, prepared for MERDC under Contract No. DA-1/4009-eng-1849.

2. California Institute of Technology, "An Investigation of the Compaction of Soil by Vibration," March 1950, prepared for MERDC under Contract No. W-44-009-eng-647.

3. Cornell University, "Soil Solidification Research;.Summarization, Fundamental and Applied Research," Vol I, September 1951, prepared for MERDC under Contract No. DA-44-009-eng-223.

4. Cornell University, "Soil Solidification Research; Fundamental Properties, Clay-Water Systems," Vol II, September 1951, prepared for MERDC under Contract No. DA-44-009-eng-223.

5.* Cornell University, "Soil Solidification Research; Applied Research, Chrome-Lignin Process and Soil Briquetting," Vol III, September 1951, prepared for MERDC under Contract No. DA-44-009-eng-223.

6. Comell University, "Production and Field Testing of 500 Tons of Chrome-Lignin Stabilized Soil Briquets," December 1952, prepared for MERDC under Contract No. DA-44-009-eng-1156.

7. Cornell University, "Studies of Mixing, Crushing, and Briquetting Equipment for Stabilized Soil," December 1953, prepared for MERDC under Contract No. DA-44-009-eng-1531.

8. Day, D. E., "Thermal Stabilization of Soils: Exploratory Laboratory Studies," Technical Report No. 6-706, Report No. 1, November 1965, U. S. Army Engineer Waterways Experiment Station, CE, Vicksburg, Miss.

9.* Eaves, Royce C., and Kozan, G. R., "Soil Stabilization: Investigation of Portland Cement as a Stabilizing Material," Technical Report No. 3-455, Report No. 8, November 1969, U. S. Army Engineer Waterways Experiment Station, $\mathrm{CE}$, Vicksburg, Miss.

10. Eustis, J. B., "Resinous Water Repellents for Soils," Technical Memorandum No. 217-1, May 1946, U. S. Army Engineer Waterways Experiment Station, CE, Vicksburg, Miss.

11. Freitag, D. R., and Decker, J. D., "Summary Reviews of Soil Stabilization Processes; Hydrated Lime and Quicklime," Miscellaneous Paper No. 3-122, Report No. 5, August 1957, U. S. Army Engineer. Waterways Experiment Station, CE, Vicksburg, Miss.

NOTE: References marked with an asterisk are actually cited in this report. The other references, though not actually cited, contain pertinent information on the soil stabilization program. 
12. Kozan, G. R., "Soil Stabilization: Field Evaluation of Calcium Acrylate (WES Test Lanes 1 and 2)," Technical Report No. 3-455, Report No. 1, June 1957, U. S. Army Engineer Waterways Experiment Station, CE, Vicksburg, Miss.

13.* Kozan, G. R., "Soil Stabilization: Initial Laboratory and Field Tests of Quicklime as a Soil Stabilizing Material," Technical Report No. 3-455, Report No. 2, August 1958, U. S. Army Engineer Waterways Experiment Station, CE, Vicksburg, Miss.

14.* Kozan, G. R., "Soil Stabilization: Investigations of a Chemically Modified Cement as a Stabilizing Material," Technical Report No. 3-455, Report No. 3, July 1960, U. S. Army Engineer Waterways Experiment Station, CE, Vicksburg, Miss.

15.* Kozan, G. R., "Soil Stabilization: Investigation of Phosphorus Pentoxide as a Soil-Stabilizing Material," Technical Report No. 3-455, Report No. 4, November 1960, U. S. Army Engineer Waterways Experiment Station, CE, Vicksburg, Miss.

16.* Kozan, G. R., and Fenwick, W. B., "Soil Stabilization: Investigations of Quicklime as a Stabilizing Material," Technical Report No. 3-455, Report No. 5, March 1962, U. S. Army Engineer Waterways Experiment Station, CE, Vicksburg, Miss.

17.* Kozan, G. R., and Fenwick, W. B., "Soil Stabilization: Investigations of a Chemically Modified Quicklime as a Stabilizing Material," Technical Report No. 3-455, Report No. 6, June 1963, U. S. Army Engineer Waterways Experiment Station, CE, Vicksburg, Miss.

18.* Kozan, G. R., and Fenwick, W. B., "Soil Stabilization: Laboratory Investigation of Soil Stabilizing Systems for Military Purposes," Technical Report No. 3-455, Report No. 7, February 1965, U. S. Army Engineer Waterways Experiment Station, $\mathrm{CE}$, Vicksburg, Miss.

19. Kozan, 'G. R., "Summary Review of Lignin and Chrome-Lignin Processes for Soil Stabilization," Miscellaneous Paper No. 3-122, Report No. 1, April 1955, U. S. Army Engineer Waterways Experiment Station, CE, Vicksburg, Miss.

20. Kozan, G. R., and Stouffer, J. D., "Summary Reviews of Soil Stabilization Processes; Mixing Principles, Techniques, and Equipment,". Miscellaneous Paper No. 3-122, Report No. 6, May 1961, U. S. Army Engineer Waterways Experiment Station, CE, Vicksburg, Miss.

21. Kozan, G. R., and Fenwick, W. B., "Summary Reviews of Soil Stabilization Processes; Electrical Stabilization of Fine-Grained Soils," Miscellaneous Paper No. 3-122, Report No. 7, October 1961, U. S. Army Engineer Waterways Experiment Station, CE, Vicksburg, Miss.

22.* Kozan, G. R., "Preliminary Investigation of Chrome-Lignin as a Stabilizing Agent in Vicksburg, Loess Soil," Miscellaneous Paper No. 3-145, September 1955, U. S. Army Engineer Waterways Experiment Station, CE, Vicksburg, Miss. 
23.* Kozan, G. R., "A Quatemary Ammonium Salt as a Stabilizing Agent in Vicksburg Loess Soil," Miscellaneous Paper No. 3-151, February 1956, U. S. Army Engineer Waterways Experiment Station, CE, Vicksburg, Miss.

24.* Kozan, G. R., and Stouffer, J. D., "Dustproofing and Waterproofing of Soils: Field and Laboratory Investigations of Selected Materials," Technical Report No. 3-530, Report No. 1, September 1959, U. S. Army Engineer Waterways Experiment Station, CE, Vicksburg, Miss.

25.* Kozan, G. R., and Stouffer, J. D., "Dustproofing and Waterproofing of Soils: Laboratory Studies of Soil Waterproofing Materials," Technical Report No. 3-530, Report No. 2, July 1963, U. S. Army Engineer Waterways Experiment Station, CE, Vicksburg, Miss.

26.* Kozan, G. R., "Investigation of Westco D-1 and D-2 Mud Control Additives," Miscellaneous Paper No. 4-735, July 1965, U. S. Army Engineer Waterways Experiment Station, CE, Vicksburg, Miss.

27.* Kozan, G. R., Ables, J. H., and Stouffer, J. D., "Investigation of Enzymatic Materials for Soil Stabilization," Miscellaneous Paper No. S-69-9, February 1969, U. S. Army Engineer Waterways Experiment Station, CE, Vicksburg, Miss.

28.* Kozan, G. R., and Stouffer, J. D., "Investigation of a Proprietary Chemical Agent for Soil Stabilization," Miscellaneous Paper S-70-1l, April 1970, U. S. Army Engineer Waterways Experiment Station, CE, Vicksburg, Miss.

29.* Massachusetts Institute of Technology, "Soil Solidification by Chemical Methods; Phase I; Literature Reviews," March 1948, prepared for MERDC under Contract No. W-44-009-eng-408.

30.* Massachusetts Institute of Technology, "Soil Solidification by Chemical Methods; Phase II, Resin Systems," March 1950, prepared for MERDC under Contract No. W-44-009-eng-408.

31.* Massachusetts Institute of Technology, "Soil Solidification by Chemical Methods; Phase III, Resin Systems," November 1951, prepared for MERDC under Contract No. DA-44-009-eng 11.

32.* Massachusetts Institute of Technology, "Soil Solidification by Chemical Methods; Phase IV, Resin Systems," November 1952, -prepared for MERDC under Contract No. DA-44-009-eng-924.

33.* Massachusetts Institute of Technology, "Soil Solidification by Chemical Methods; Phase V, Resin Systems," November 1953, prepared for MERDC under Contract No. DA-44-009-eng-1494.

34.* Massachusetts Institute of Technology, "Soil Solidification by Chemical Methods; Phase VI, Resin Systems," November 1954, prepared for MERDC under Contract No. DA-44-009-eng-2002.

35.* Massachusetts Institute of Technology, "Soil Solidification by Chemical Methods; Phase VII, Resin Systems," November 1955, prepared for MERDC under Contract No. DA-22-079-eng-171. 
36.* Massachusetts Institute of Technology, "Soil Stabilization by Chemical Methods; Phase VIII, Asphalts, Phosphorus Compounds, Cement, Resins, Trace Chemicals," November 1956, prepared for the U. S. Army Engineer Waterways Experiment Station under Contract No. DA-22-079-eng-171.

37.* Massachusetts Institute of Technology, "Soil Stabilization by Chemical Methods; Phase IX, Asphalts, Phosphorus Compounds, Cement, Resins, Trace Chemicals," November 1957, prepared for the U. S. Army Engineer Waterways Experiment Station under Contract No. DA-22-079-eng-171.

38.* Massachusetts Institute of Technology, "Soil Stabilization by Chemical Methods; Phase X, Cement, Phosphorus Compounds, Asphalt Emulsions, Trace Chemicals," November 1958, prepared for the U. S. Army Engineer Waterways Experiment Station under Contract No. DA-22-079-eng-171.

39.* Massachusetts Institute of Technology, "Soil Stabilization by Chemical Methods; Phase XI, Cement, Phosphorus Compounds, Sodium Silicates, Asphalt Emulsions," November 1959, prepared for the U. S. Army Engineer Waterways Experiment Station under Contract No. DA-22-079-eng-171.

40.* Massachusetts Institute of Technology, "Soil Stabilization by Chemical Methods; Phase XII, Cement, Lime, Phosphorus Compounds, Sodium Silicates, Asphalt Emulsions," November 1960, prepared for the U. S. Army Engineer Waterways Experiment Station under Contract No. DA-22-079-eng-171.

41.* Massachusetts Institute of Technology, "Soil Stabilization by Chemical Methods; Phase XIII, Phosphorus Compounds, Lime, Asphalt Emulsions, and Surmary Evaluation of Potential Systems," November 1961, prepared for the U. S. Army Engineer Waterways Experiment Station under Contract No. DA-22-079-eng-288.

42. Mitchell, J. K., "Summary Reviews of Soil Stabilization Processes; Calcium Acrylate Treatment," Miscellaneous Paper No. 3-122, Report No. 2, January 1956, U. S. Army Engineer Waterways Experiment Station, CE, Vicksburg, Miss.

43. Mitchell, J. K., "Summary Reviews of Soil Stabilization Processes; Soil-Cement," Miscellareous Paper No. 3-122, Report No. 3, September 1956, U. S. Army Engineer Waterways Experiment Station, $\mathrm{CE}$, Vicksburg, Miss.

44. Mitchell, J. K., "Summary Reviews of Soil Stabilization Processes; Bituminous Treatment," Miscellaneous Paper No. 3-122, Report No. 4, November 1956, U. S. Army Engineer Waterways Experiment Station, CE, Vicksburg, Miss.

45. Obert, Leonard, and Blair, Byron, "Solidifying or Stabilizing Soils for Military Operations by Freezing," August 1948, prepared for MERDC by the U. S. Bureau of Mines under Project No. AC-697. 
46. Reissner, Eric, "Analytical Studies of the Action of Thin Flexible Surfaces under Load over Flexible Subgrades," January 1954, prepared for MERDC under Contract No. DA-44-009-eng-1863 with the Massachusetts Institute of Technology.

47. Reynolds, J. H., Jr., "Evaluation of Chrome-Lignin Stabilized Soil Briquets as Traffic-Bearing Media," Report No. 1326, October 1963, MERDC, Ft. Belvoir, Va.

48. Rodes, V. H., "First Interim Report, Solidifying or Stabilizing Soils for Military Operations," Report 1095, December 1948, MERDC, Ft. Belvoir, Va.

49. Rodes, V. H., and Reynolds, J. H., Jr., "Second Interim Report: Solidifying or Stabilizing Soils for Military Operations," Report No. 1306, December 1954, MERDC, Ft. Belvoir, Va.

50. Sanders, G. S., "Development of Aerial Dispersal System for RapidLanding Site Stabilization," Contract Report No. 3-169, September 1967, prepared for the U. S. Army Engineer Waterways Experiment Station under Contract No. DA-22-079-eng-490.

51.* Stouffer, J. D., "Dustproofing and Waterproofing of Soils: Investigation of Aniline-Furfural Resin as a Dustproofer and Waterproofer for Two Clay Soils," Technical Report No. 3-350, Report No. 3, U. S. Army Engineer Waterways Experiment Station, CE., Vicksburg, Miss.

52. Tauxe, G. J., O'Brien, P. F., and Young, G. B. W., "Summary of Literature Survey on Soil Stabilization with Cement," July 1947, prepared for MERDC under Contract No. W-44-009-eng-438.

53. Tauxe, G. J., O'Brien, P. F., and Young, G. B. W., "Surmary of Literature Survey of Soil Stabilization with Resinous Materials," prepared for MERDC under Contract No. W-44-009-eng-438.

54. Tauxe, G. J., O'Brien, P. F., and Young, G. B. W., "Engineering Soil Solidification Research: Sumary of Literature Survey of Soil Stabilization," February 1948, prepared for MERDC under Contract No. W-44-009-eng-438.

55. Tauxe, G. J., O'Brien, P. F., and Young, G. B. W., "Soil Stabilization Bibliography," December 1947, prepared under Contract No. W-44-099-eng-438 for MERDC.

56. Tauxe, G. J., O'Brien, P. F., and Young, G. B. W., "Summary of Literature Survey on Soil Stabilization by Thermal Methods," January 1948, prepared for MERDC under Contract No. W-44-009-eng438.

57.* Impola, C. N., and Olsen, D. A., "Research Study on Soil Treatment Materials for Dust Palliation, Soil Waterproofing, and Soil Strengthening," Contract Report S-68-5, November 1968, prepared for the U. S. Army Engineer Waterways Experiment Station under Contract No. DA-22-079-eng-437. 
58. Mitchell, J. K., Shen, Chik-Kang, and Monismith, C. L., "Behavior of Stabilized Soils under Repeated Loading," Contract Report No. 3-145, Report No. 1, December 1965, "Background, Equipment, Preliminary Investigations, Repeated Compression and Flexure Tests on Cement-Treated Silty Clay," prepared for the U. S. Army Engineer Waterways Experiment Station under Contract No. DA-22-079-eng-414.

59. Mitchell, J. K., and Monismith, C. L., "Behavior of Stabilized Soils under Repeated Loading," Contract Report No. 3-145, Report No. 2, September 1966, "Behavior in Repeated Flexure; Frequency and Duration Effects; Fatigue Failure Analyses," prepared for the U. S. Army Engineer Waterways Experiment Station under Contract No. DA-22-079-eng-414.

60. Mitchell, J.K., Fossberg, P. E., and Monismith, C. L., "Behavior of Stabilized Soils under Repeated Loading," Contract Report No. 3-145, Report No. 3, May 1969, "Repeated Compression and Flexure Tests on Cement- and Lime-Treated Buckshot Clay Confining Pressure Effects in Repeated Compression for Cement-Treated Silty Clay," prepared for the U. S. Army Engineer Waterways Experiment Station under Contract No. DA-22-079-eng-414.

61. Wang, M. C., Mitchell, J. K., and Monismith, C. L., "Behavior of Stabilized Soils under Repeated Loading," Contract Report No. 3-145, Report No. 4, October 1970, "Stresses and Deflections in Cement-Stabilized Pavements," prepared for the U. S. Army Engineer Waterways Experiment Station under Contract No. DA-22-079-eng-414.

62. Mitchell, J. K., Ueng, T-S, and Monismith, C. L., "Behavior of Stabilized Soils under Repeated Loadings," Contract Report No. 3-145, Report No. 5, "Performance Evaluation of Cement-Stabilized Soil Layers and Its Relationship to Pavement Design," prepared for the U. S. Army Engineer Waterways Experiment Station under Contract No. DA-22-079-eng-414.

63. Mitchell, J. K., Dzwilewski, Peter, and Monismith, C. L., "Behavior of Stabilized Soils under Repeated Loadings," Contract Report No. 3-145, Report No. 6, October 1974, "A Summary Report with a Suggested Structural Pavement Design Procedure," prepared for the U. S. Army Engineer Waterways Experiment Station under Contract No. DA-22-079-eng-414.

64. Esrig, M. I., "Feasibility Study of Electrokinetic Processes for Stabilization of Soils for Military Mobility Purposes," Contract Report No. 3-73, Report No. 1, May 1964, "A Theoretical Study of the Equations Governing Electroosmotic Flow and a Laboratory Investigation of the Effects of Electrokinetic Treatment on an Illitic Soil," prepared for the U. S. Army Engineer Waterways Experiment Station under Contract No. DA-22-079-eng-346. 
65. Esrig, M. I., and Maftenyi, S., "A Feasibility Study of Electrokinetic Processes for Stabilization of Soils for Military Mobility Purposes," Contract Report No. 3-73, Report No. 2, June 1965, "An Analysis of the Electroosmotic Phenomenon in Soil Capillary Systems," prepared for the U. S. Army Engineer Waterways Experiment Station under Contract No. DA-22-079-eng-346.

66. Esrig, M. I., "A Feasibility Study of Electrokinetic Processes for Stabilization of Soils for Military Mobility Purposes," Contract Report No. 3-73, Report No. 3, August 1966, "Results of a Preliminary Field Investigation," prepared for the U. S. Army Engineer Waterways Experiment Station under Contract No. DA-22079-eng-346.

67. Esrig, M. I., "A Feasibility Study of Electrokinetic Processes for Stabilization of Soils for Military Mobility Purposes," Contract Report No. 3-73, Report No. 4, July 1967, "Laboratory Investigation of Electrokinetic Treatment of Consolidated Soils, prepared for the U. S. Army Engineer Waterways Experiment Station under Contract No. DA-22-079-eng-346.

68. Esrig, M. I., "A Feasibility Study of Electrokinetic Processes for Stabilization of Soils for Military Mobility Purposes," Contract No. 3-73, Report No. 5, March 1968, "A Study of Pore Water Pressures during Electrokinetic Treatment," prepared for the U. S. Army Engineer Waterways Experiment Station under Contract No. DA-22-079-eng-346.

69. Massachusetts Institute of Technology, "Soil Stabilization," Contract Report No. 3-63, Phase Report I; May 1963, "Engineering Behavior of Partially Saturated Soils," prepared for the U. S. Army Engineer Waterways Experiment Station under Contract No. DA-22-079-eng-288.

70. Massachusetts Institute of Technology, "Soil Stabilization," Contract Report No. 3-63, Phase Report No. 2, September 1963, "Triaxial Equipment and Computer Program for Measuring the Strength Behavior of Stabilized Soils," prepared for the U. S. Army Engineer Waterways Experiment Station under Contract No. DA-22079-eng-288.

71. Wissa, A. E. Z., and Ladd, C. C., "Soil Stabilization," Contract Report No. 3, Phase Report No. 3, July 1964, "Effective StressStrength Behavior of Compacted Stabilized Soils," prepared for the U. S. Army Engineer Waterways Experiment Station under Contract No. DA-22-079-eng-288.

72. Wissa, A. E. Z., and Halaby, Rurik, "Soil Stabilization," Contract Report No. 3-63, Phase Report No. 4, October 1964, "Chemical Stabilization of Selected Tropical Soils from Puerto Rico and Panama," prepared for the U. S. Army Engineer Waterways Experiment Station under Contract No. DA-22-079-eng-288. 
73. Wissa, A. E. Z., and Ladd, C. C., "Soil Stabilization," Contract Report No. 3-63, Phase Report No. 5, June 1965, "Shear Strength Generation in Stabilized Soils," prepared for the U. S. Army Engineer Waterways Experiment Station under Contract No. DA-22-079eng-288.

74. Wissa, A. E. Z., and Monti, R. P., "Soil Stabilization," Contract Report No. 3-63, Phase Report No. 6, "Compressibility-Permeability Behavior of Untreated and Cement-Stabilized Clayey Silt," prepared for the U. S. Army Engineer Waterways Experiment Station under Contract No. DA-22-079-eng-465.

75. Wissa, A. E. Z., and Paniagua, J. G., "Soil Stabilization," Contract No. 3-63, Phase Report No. 7, June 1969, "A Durability Test for Stabilized Soils," prepared for the U. S. Army Engineer Waterways Experiment Station under Contract No. DA-22-079-eng-465.

76. Wissa, A. E. Z., Ferferbaum-Zyto, S., and Paniagua, J. G., "Soil Stabilization," Contract Report No. 3-63, Phase Report No. 8, January 1970, "Effect of Molding Conditions on the Effective Stress-Strength Behavior of a Stabilized Clayey Silt," prepared for the U. S. Army Engineer Waterways Experiment Station under Contract No. DA-22-079-eng-465.

77. Wissa, A. E. Z., McGillivray, R. T., and Paniagua, J. G., "Soil Stabilization," Contract Report No. 3-63, Phase Report No. 9, August 1971, "The Effects of Mixing Conditions, Method of Compaction, and Curing Conditions on the Effective Stress-Strength Behavior of a Stabilized Soil," prepared for the U. S. Army Engineer Waterways Experiment Station under Contract No. DA-22079-eng-465.

78. Wissa, A. E. Z., and Paniagua, J. G., "Soil Stabilization," Contract Report No. 3-63, Phase Report No. 10, June 1972, "Equipment for Studying the Effect of Repeated Loading on the StressStrength Behavior of Stabilized Soils," prepared for the U. S. Army Engineer Waterways Experiment Station under Contract No. DA-22079-eng-465. 
Table 1

EFFECTIVE SOIL STABILIZERS

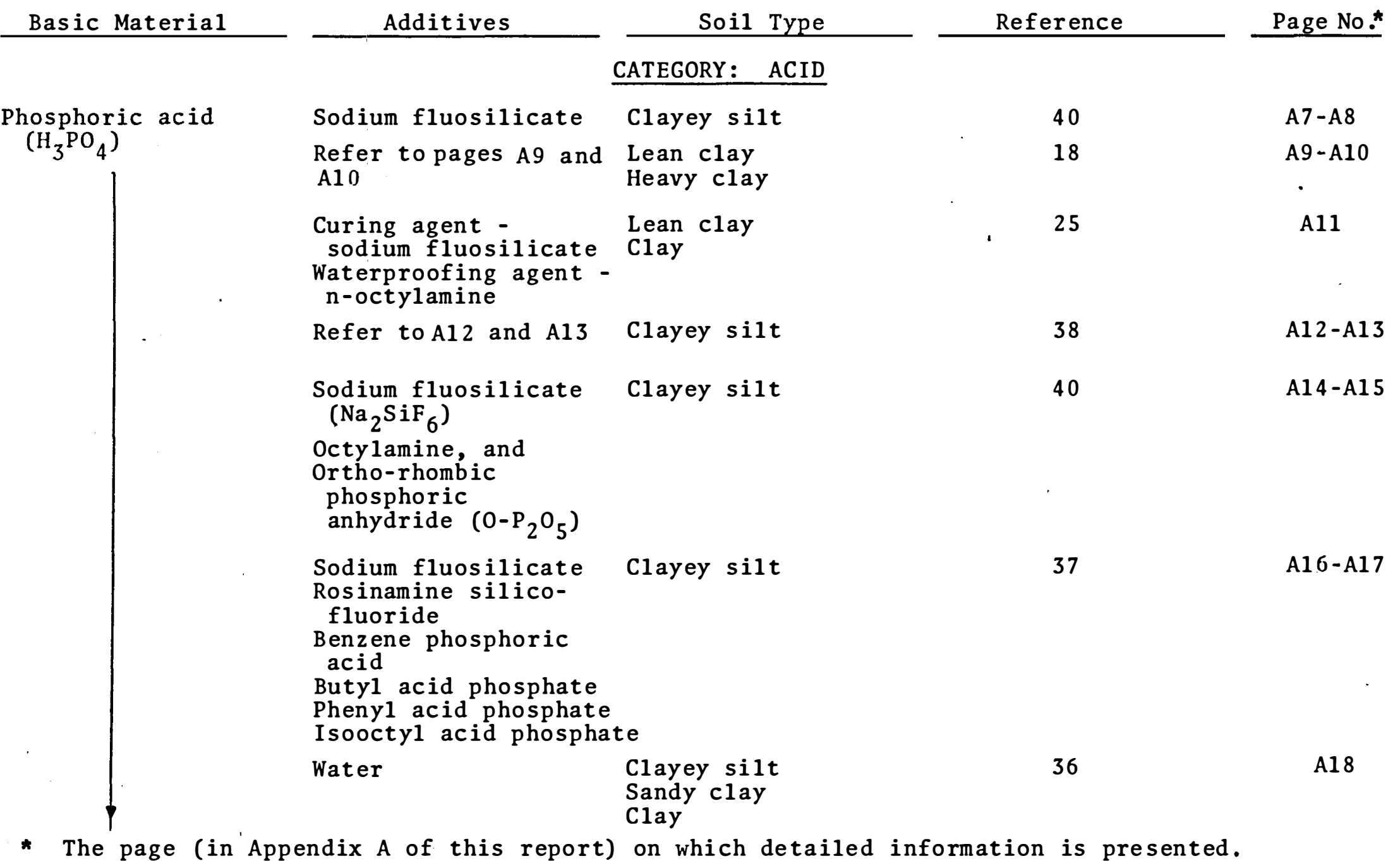


Table 1 (Cont'd)

\begin{tabular}{|c|c|c|c|c|}
\hline Basic Material & Additives & Soil Type & Reference & Page No. \\
\hline \multirow[t]{3}{*}{$\begin{array}{l}\text { Phosphorus } \\
\text { pentoxide }\end{array}$} & \multirow{3}{*}{$\begin{array}{l}\text { Sodium } \\
\quad \text { fluosilicate }\end{array}$} & $\begin{array}{l}\text { Sandy silt, clayey } \\
\text { silt, sandy clay, } \\
\text { loess, and clay }\end{array}$ & 36 & A19 \\
\hline & & \multirow[b]{2}{*}{ CATEGORY: ASPHALT } & 15 & A20 \\
\hline & & & & $\cdot$ \\
\hline $\begin{array}{l}\text { Asphalt cutback } \\
\text { (Refer to page A23) }\end{array}$ & $\begin{array}{l}\text { Phosphorus } \\
\text { pentoxide }\left(\mathrm{P}_{2} \mathrm{O}_{5}\right)\end{array}$ & Clayey silt & 37 & A23 \\
\hline $\begin{array}{l}\text { Aspha1t cutback } \\
\text { (50-60 pen) }\end{array}$ & $\begin{array}{l}\text { Solvents (refer } \\
\text { to pages A24-A25) } \\
\text { Phosphorus } \\
\text { pentoxide }\left(\mathrm{P}_{2} \mathrm{O}_{5}\right)\end{array}$ & Clayey silt & 37 & $\mathrm{~A} 24-\mathrm{A} 25$ \\
\hline $\begin{array}{l}\text { Asphalt cutback } \\
\text { (Refer to pages A26 } \\
\text { and A27) }\end{array}$ & $\begin{array}{l}\text { Phosphorus } \\
\text { pentoxide }\left(\mathrm{P}_{2} \mathrm{O}_{5}\right)\end{array}$ & Clayey silt & 37 & $\mathrm{~A} 26-\mathrm{A} 27$ \\
\hline $\begin{array}{l}\text { Cutback asphalt } \\
\text { (Straight run, } \\
\text { cracked, and } \\
\text { blown) }\end{array}$ & $\begin{array}{l}\text { Refer to A28 and } \\
\text { A29 }\end{array}$ & Clayey silt & 35 & $\mathrm{~A} 28-\mathrm{A} 29$ \\
\hline \multirow[t]{2}{*}{$\begin{array}{l}\text { Cutback asphalt } \\
\text { ( } 40-50 \text { pen } \\
\text { straight run } \\
\text { asphalt) }\end{array}$} & Refer to $A 30$ & $\begin{array}{l}\text { Lean clay and } \\
\text { heavy clay }\end{array}$ & 25 & A30 \\
\hline & $\begin{array}{l}\text { Solvent - unleaded } \\
\text { gasoline } \\
\text { Phosphoric acid } \\
\left(\mathrm{H}_{3} \mathrm{PO}_{4}\right)\end{array}$ & Lean clay and clay & 25 & A31 \\
\hline
\end{tabular}


Table 1 (Cont'd)

\begin{tabular}{|c|c|c|c|c|c|c|}
\hline \multicolumn{3}{|c|}{ Basic Material } & Additives & Soil Type & \multirow{2}{*}{$\frac{\text { Reference }}{25}$} & \multirow{2}{*}{$\frac{\text { Page No. }}{A 37}$} \\
\hline $\begin{array}{l}\text { Cutback } \\
50 \text { pen } \\
\text { aspha } 1 t \text { ) }\end{array}$ & $\begin{array}{l}\text { asphalt } \\
\text { straight } \\
\text { ) }\end{array}$ & $\begin{array}{l}(40- \\
\text { run }\end{array}$ & $\begin{array}{l}\text { Solvent - unleaded } \\
\text { gasoline } \\
\text { Additive - } \\
\text { phosphorus pentoxide } \\
\left(\mathrm{P}_{2} \mathrm{O}_{5}\right)\end{array}$ & Lean clay and clay & & \\
\hline & & & $\begin{array}{l}\text { Solvent - unleaded } \\
\text { gasoline } \\
\text { Additives (Refer } \\
\text { to A38-A39) }\end{array}$ & Lean clay and clay & 25 & A38 \\
\hline & & & $\begin{array}{l}\text { Solvent - unleaded } \\
\text { gasoline } \\
\text { Additives (Refer to } \\
\text { A40-A41) }\end{array}$ & Lean clay and clay & 25 & A40-A41 \\
\hline & & & $\begin{array}{l}\text { Solvent - unleaded } \\
\text { gasoline } \\
\text { Additives (Refer to } \\
\text { A40-A41) }\end{array}$ & Lean clay and clay & 25 & $\mathrm{~A} 42-\mathrm{A} 43$ \\
\hline . & & & $\begin{array}{l}\text { Solvent - unleaded } \\
\text { gasoline } \\
\text { Additives (Refer to } \\
\text { A42-A43) }\end{array}$ & Lean clay and clay & 25 & A $44-A 45$ \\
\hline $\begin{array}{r}\text { Straight } \\
\text { asphalt }\end{array}$ & run & & Refer to A46-A47 & Clayey silt & 36 & A44-A45 \\
\hline $\begin{array}{l}\text { Straight } \\
\text { asphalt } \\
\text { pen) }\end{array}$ & $=\operatorname{run}_{(40-50}$ & 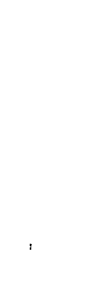 & $\begin{array}{l}\text { Emulsifying agents - } \\
\text { Duomeen T and } \\
\text { hydrochloric acid } \\
\text { Solvent - gasoline } \\
\text { Additive - chromic } \\
\text { chloride and } \\
\text { phosphoric acid }\end{array}$ & Clayey silt & 40 & A48 \\
\hline
\end{tabular}


Table 1 (Cont'd)

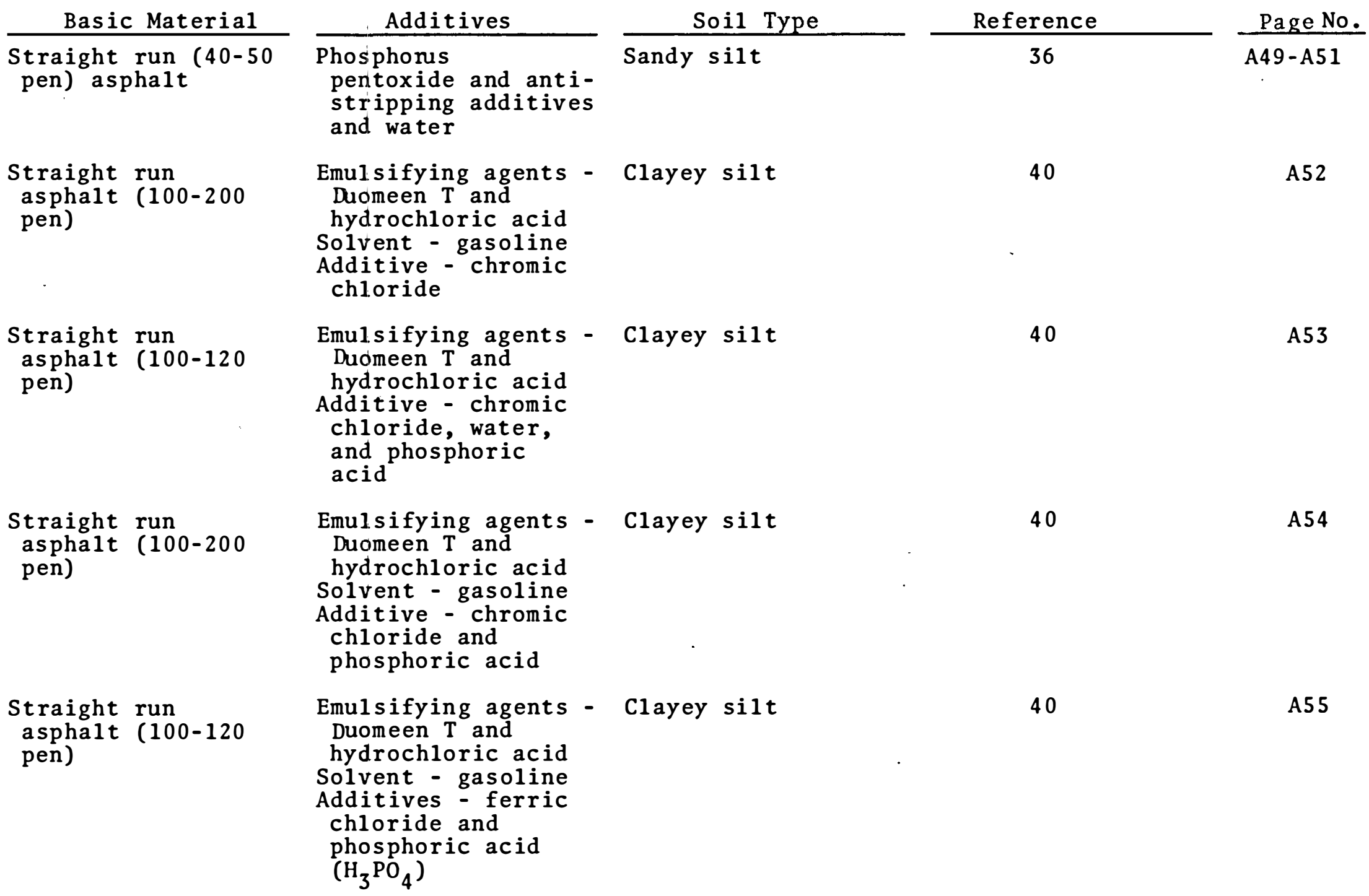


Table 1 (Cont'd)

Basic Material

Straight run
asphalt $(100-120$
pen)
pen)

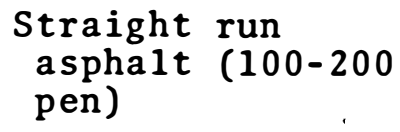

Alumina cement

\section{Cement}

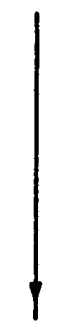

Additives

Emulsifying agents - Clayey silt

Duomeen $T$ and

hydrochloric acid

Solvent - gasoline

and phosphoric

acid

Emulsifying agent -

nonic 218

Solvent - gasoline

and phosphoric

acid $\left(\mathrm{H}_{3} \mathrm{PO}_{4}\right)$

Emulsifying agents Duomeen $T$ and

hydrochloric acid

Solvent - gasoline

Additives - ferric

chloride and

phosphoric acid

\section{CATEGORY: CEMENT}

\section{Modifiers (Refer to A62-A63)}

Sodium hydroxide plus sodium sulfate

Sodium hydroxide $(\mathrm{NaOH})$

Sodium hydroxide plus sodium sulfate

\section{Loess}

Clay (Texas \#2)

Clay (Vicksburg)

Sand (Wisconsin \#1)

Reference

Page No.

A57

40

A58

40

A5 9

Internal Data (1956), not published

39

40

39
A62-A63

A64

A65

A66 
Table 1 (Cont'd)

Basic Material

Cement

Cement (plus 1N

$\mathrm{NaOH}$ - sodium

hydroxide)

Fast Fix

Lumnite cement

Plaster of Paris

Portland cement
Additives

Sodium hydroxide plus sodium sulfate

Refer to $A 68-A 69$
Reference

39

Page No.

A67

Clay (Vicksburg)

40

A68-A69

Lean clay, heavy clay, and sand

Sand, loess, and heavy clay

Lean clay and heavy clay

Loess

Lean clay and clay

Clayey silt

Clay (Texas \#2)

Clay (Vicksburg)

Silt

Sand (Wisconsin \#2)
Internal Data (1971), A70 not published

Internal Data (1956), A72-A73 not published

Internal Data (19561957), not published 
Table 1 (Cont'd)

Basic Material Portland cement

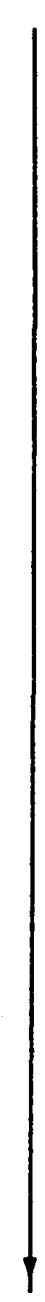

Additives

Refer to pages A87A8 8

Dispersants (Refer

to pages $A 89-A 90)$

Sodium hydroxide

Sodium carbonate

Sodium metasilicate

Sodium hydroxide

Sodium carbonate

Sodium metasilicate

Sodium sulfate

Sodium hydroxide, sodium carbonate, sodium metasilicate, sodium sulfate, sodium aluminate, sodium fluosilicate, sodium floride, sodium fluoborate, and sodium tetraborate

Sodium hydroxide, Silt sodium sulfate, sodium aluminate

Sodium hydroxide, sodium sulfate, sodium aluminate, ferric chloride plus sodium hydroxide, octylamine plus sodium hydroxide

Silt

Soil Type
Lean clay and heavy
clay

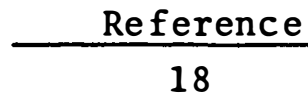

35

A89-A90

Clayey silt

Loess

38

A91-A92

Sand (Wisconsin \#1)

38

A93-A94

A95-A96

A97-A98

Clay (Texas \#2)

A99-A100 $\frac{\text { Page No. }}{A 87-A 88}$ 
Table 1 (Cont'd )

Basic Material

Portland cement
Additives

Sodium hydroxide

sodium sulfate,

sodium aluminate,

sodium metasilicate

Sodium. hydroxide sodium sulfite, sodium carbonate

Sodium hydroxide, sodium sulfite, sodium carbonate, sodium metasilicate

Sodium hydroxide, sodium hydroxide plus barium chloride, sodium sulfite, sodium carbonate, sodium metasilicate

Sodium metasilicate

Sodium orthosilicate

Sodium

orthosilicate, sodium metasilicate, grade 50 silicate, grade 40 silicate, sodium oxide $\left(\mathrm{Na}_{2} \mathrm{O}\right)$, silicon dioxide $\left(\mathrm{SiO}_{2}\right)$

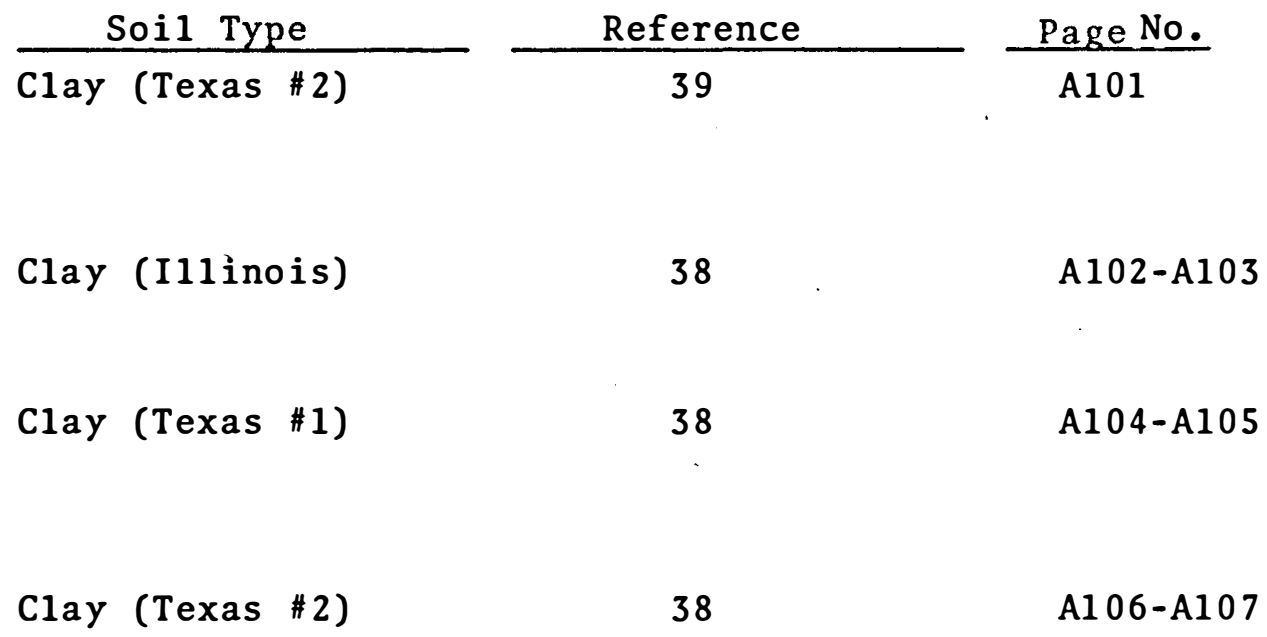


Table 1 (Cont'd)

Basic Material

Portland cement

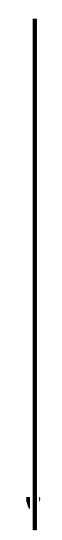

Type I normal

portland cement

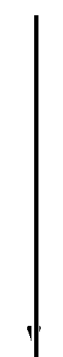

Type I portland

cement
Additives

$$
\begin{aligned}
& \text { Sodium sulfate } \\
& \text { (Also refer to } \\
& \text { pages A112-Al13) }
\end{aligned}
$$

Sodium sulfate,

ET-224 dispersant

barium chloride,

sodium fluosilicate

Sodium sulfate and sodium metasilicate

Sulfate compounds (Refer to pages A117-A118)

Chemical additives (Refer to pages

A119-A121)

Chemical additives (Refer to pages A122-A124)

Chemical additives (Refer to pages A125-A127)

Sodium hydroxide (with heavy clay only)
Reference

Page No.

14

A112-A113

Loess

A114-A115

Lean clay and clay

25

A116

Sand (Wisconsin \#1)

Loess

A119-A121

Silt

Silty clay

A125-A127

Lean clay and heavy clay 
Table 1 (Cont'd)

\begin{tabular}{|c|c|c|c|c|}
\hline Basic Material & Additives & Soil Type & Reference & Page \\
\hline & v & CATEGORY: LIME & & \\
\hline Hydrated lime & $\begin{array}{l}\text { Sodium hydroxide, } \\
\text { sodium sulfate, } \\
\text { sodium carbonate, } \\
\text { magnesium sulfate, } \\
\text { calcium oxide, } \\
\text { calcium hydroxide, } \\
\text { portland cement }\end{array}$ & Lean clay & $\begin{array}{l}\text { Internal Data }(1960) \text {, } \\
\text { not pulbished }\end{array}$ & $A 132$ \\
\hline \multirow{2}{*}{$\begin{array}{l}\text { Calcium hydroxide } \\
\text { (slaked lime) }\end{array}$} & & Clay (Vicksburg) & 41 & A133 \\
\hline & Magnesium sulfate & Clay. (Vicksburg) & 41 & A134 \\
\hline $\begin{array}{l}\text { Calcium and } \\
\text { magnesium limes } \\
(\mathrm{CaO} \text { and } \mathrm{MgO})\end{array}$ & Magnesium sulfate & $\begin{array}{l}\text { Lean clay and heavy } \\
\text { clay }\end{array}$ & $\begin{array}{l}\text { Internal Data (1961), } \\
\text { not published }\end{array}$ & A135-A136 \\
\hline Calcium oxide & & Clay (Houston black) & 41 & $A 137$ \\
\hline $\begin{array}{l}\text { Calcium oxide } \\
\text { (lime) }\end{array}$ & & Clay (Vicksburg) & 41 & A138 \\
\hline \multirow[t]{2}{*}{ Calcium oxide } & $\begin{array}{l}\text { Refer to pages } \\
\text { A139-A140 }\end{array}$ & Clay (Houston black) & 41 & $\mathrm{~A} 139-\mathrm{A} 140$ \\
\hline & $\begin{array}{l}\text { Refer to pages } \\
\text { A141-A142 }\end{array}$ & Clay (Vicksburg) & 41 & $\mathrm{~A} 141-\mathrm{A} 142$ \\
\hline $\begin{array}{l}\text { Calcium oxide } \\
\text { (lime) }\end{array}$ & Magnesium sulfate & Clay (Vicksburg) & 41 & A143 \\
\hline Calcium oxide & $\begin{array}{l}\text { Magnesium sulfate, } \\
\text { potassium sulfate, } \\
\text { magnesium chloride }\end{array}$ & Clay (Vicksburg) & 40 & $\mathrm{~A} 144-\mathrm{A} 145$ \\
\hline
\end{tabular}


Table 1 (Cont'd)

\section{Basic Material}

\section{Calcium oxide plus} magnesium sulfate plus cutback asphalt

\section{Quicklime}

$$
\mid
$$

AM9 (watersoluble acrylamide and diacrylamide)

Aniline-furfural

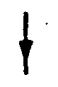

Additives

Solvent - gasoline

$\frac{\text { Soil Type }}{\text { Lean clay and clay }}$

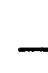

Reference

25

Clayey silt, silt, clay, and loess

17

Magnesium sulfate

$$
\begin{aligned}
& \text { Lean clay, heavy } \\
& \text { clay, clayey silt, } \\
& \text { silt, blue clay, } \\
& \text { sandy clay, and } \\
& \text { sand } \\
& \text { Lean clay } \\
& \text { Lean clay and heavy } \\
& \text { clay }
\end{aligned}
$$

18

to pages A153-

A154)

\section{CATEGORY: RESIN}

\section{Catalyst -}

dime thylamino-

propionitrile-

potassium

ferricyanide-

ammonium

persulfate

Solvent - water
Sand
Page No.

A146

A $147-A 148$

A149-A150

A151-A152

A1 53-A154 
Table 1 (Cont'd)

Basic Material Aropol 7110

Arothane 170

Bisphenol A

(Epon 828)

Calcium acrylate.

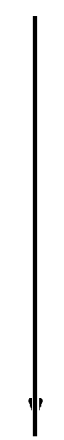

Epon VIII

Epon 562
Additives

Solvent - styrene

Solvent - butyl acetate

Catalyst - Ashland

\#1496

Solvent - solox

$$
\text { Va }
$$

Curing agents -
Agent A (amine)

diethylenetriamine

(Refer to pages

A169-A170)

Water

\begin{tabular}{|c|c|}
\hline & Loess \\
\hline $\begin{array}{l}\text { Refer to pages A164- } \\
\text { A165 }\end{array}$ & Sandy \\
\hline $\begin{array}{l}\text { Salt additives } \\
\text { (Refer to page. } \\
\text { A166 }\end{array}$ & Sandy \\
\hline $\begin{array}{l}\text { Various salts } \\
\text { (Refer to A167-A168 }\end{array}$ & Sandy \\
\hline $\begin{array}{l}\text { Curing agents - } \\
\text { Agent A (amine) } \\
\text { diethylenetriamine } \\
\text { (Refer to pages } \\
\text { Al69-A170) } \\
\text { Water }\end{array}$ & Sandy \\
\hline $\begin{array}{l}70 \% \text { diethylene } \\
\text { triamine, } 30 \% \\
\text { dimethyl aminomethyl } \\
\text { phenol (above curing } \\
\text { agents); solvent - } \\
\text { acetone; potassium } \\
\text { hydroxide (KOH) }\end{array}$ & Sandy \\
\hline
\end{tabular}

hydroxide ( $\mathrm{KOH})$
Reference
57

57

57

24

31

31

$\frac{\text { Page No. }}{A 160}$

A161

A162

A163

A164-A165

A166

A167-A168

37

A171 
Table 1 (Cont'd)

Basic Material

Epon 828

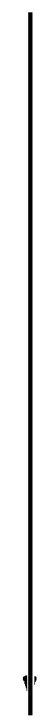

Epon 834

Arquad 2HT

(Dialkyl

dimethyl -

ammonium

chloride)
Additives

\section{Xylene}

Curing agents -

diethylene triamine,

diethylaminomethyl

phenol, mixtures of

above curing agents, polyethylene

$70 \%$ diethylene

triamine, $30 \%$

dimethyl aminomethyl

phenol (curing

agents); solvents -

Refer to pages

A173-A174

Curing agent - 7:1 ratio of diethylene clay
Soil Type

Sandy clay

Sandy clay

37

35
Page No.

A172

A173-A174

triamine to dimethyl

aminomethyl phenol

Curing agents -

tetraethylenepentamine

diethylenetriamine

Water

Refer to pages A176-

A 177

\section{CATEGORY: SALT}

Loess

24

A180 
Table 1 (Cont'd)

Basic Material

Sodium silicate ( $30 \%$ solution) Sodium silicate plus
basic magnesium

carbonate

\section{Sodium silicate $\mathrm{N}$}

Sodium silicate

(composed of

$1.59 \%$ sodium oxide and $3.82 \%$ silicon

dioxide)

\section{Sodium silicate \\ (49.8\% solids, potassium oxide to silicon \\ dioxide $=$ \\ $1: 1.58$ )}

\section{Sodium silicate}

$$
\begin{aligned}
& \text { Sodium silicate } \\
& \text { ( } 49.8 \% \text { solids; } \\
& \text { sodium oxide to } \\
& \text { silicon dioxide }= \\
& 1: 1.58)
\end{aligned}
$$

Soil Type

Reference

Page No.

\section{CATEGORY: SILICATE}

Loess

24

A183

Loess

A184-A185

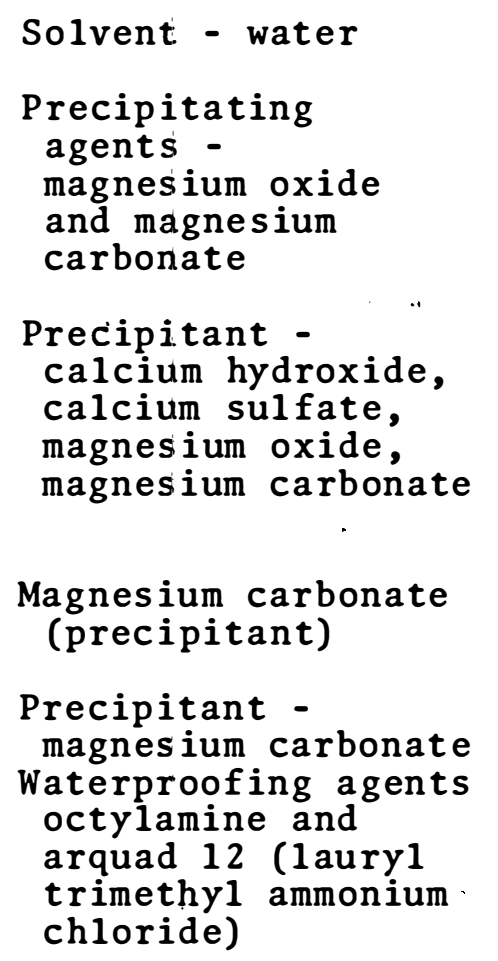

Precipitant calcium hydroxide, calcium sulfate, magnesium oxide, magnesium carbonate

Magnesium carbonate (precipitant)

Clayey silt

A190-
Clayey silt

\section{A186}

A187-A188

A189 
Table 1 (Cont'd)

Basic Material

Sodium silicate plus calcium hydroxide, $\mathrm{Ca}(\mathrm{OH})$

Sodium silicate plus basic magnesium

carbonate

Chrome lignin

Lignin (clarion extract)

Powder A plus

powder $B$

SA-1

Sandcrete

Sodium methylethyl propyl siliconate

Soil-Set
Additives

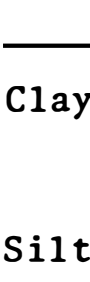

Soil Type

layey silt

Silt

\section{CATEGORY: OTHER}

\section{Loess}

Clay

Sodium dichromate sulfuric acid, sodium chloride

Loess and heavy clay

Lean clay and heavy clay

Lean clay and sand

Loess

Lean clay, heavy
Reference

40

Page No.

A193

A194-A195

A197

5

A198-A199

Internal Data (1974)

A200

not published

Internal Data (1974), A201-A202 not published

Internal Data (1972), not published

24

A204

Internal Data (1966), A205-A206 not published 
Table 2

NONEFFFCTIVE SOIL STAB'TLI ZERS

Basic Material
Amine D acetate
Octadecyl amine acetate
Resyn $78-1035$ (polyvinyl
acetate emulsion)
Rosin amine D acetate
Seycorez B-17
Hydrochloric acid
Nitric acid
Orthorhombic
phosphorus
pentoxide
Orthorhombic
phosphorus pentoxide
O-P 20
Orthorhombic

\begin{tabular}{|c|c|c|c|c|c|}
\hline \multicolumn{2}{|l|}{ Additives } & \multicolumn{4}{|c|}{ Soil Type } \\
\hline CATEGORY: & ACETATE & & & & \\
\hline & & Lean & clay & and & clay \\
\hline & & Lean & clay & and & clay \\
\hline & & Clay & & & \\
\hline & & Clay & & & \\
\hline & & Lean & clay & & \\
\hline
\end{tabular}

Reference

CATEGORY: ACID

\begin{tabular}{|c|c|}
\hline Water & Clay \\
\hline Water & Clay \\
\hline $\begin{array}{l}\text { Cure agent - sodium } \\
\text { fluosilicate }\end{array}$ & Lean clay and clay \\
\hline $\begin{array}{l}\text { Curing agent - sodium } \\
\text { fluosilicate } \\
\text { Waterproofing agent - } \\
\text { n-octylamine }\end{array}$ & Lean clay and clay \\
\hline $\begin{array}{l}\mathrm{O}-\mathrm{P}_{2} \mathrm{O}_{5}, \mathrm{Na}_{2} \mathrm{SiF}_{6} \text { (sodium } \\
\text { fluosilicate), n-octylamine }\end{array}$ & $\begin{array}{l}\text { Lean clay and heavy } \\
\text { clay }\end{array}$ \\
\hline
\end{tabular}


Table 2 (Cont'd)

Basic Material

Phosphoric acid

Phosphoric acid $\left(\mathrm{H}_{3} \mathrm{PO}_{4}\right)$

Phosphoric acid $\left(\mathrm{H}_{3} \mathrm{PO}_{4}\right)$

Phosphoric acid

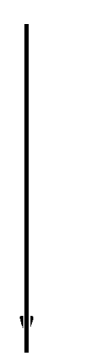

Phosphoric acid $\left(\mathrm{H}_{3} \mathrm{PO}_{4}\right)$

Phosphoric acid

Phosphoric acid $\left(\mathrm{H}_{3} \mathrm{PO}_{4}\right)$ Curing agent - sodium fluosilicate

Waterproofing agent -

n-octylamine,

orthorhombic phosphorus

pentoxide

\begin{tabular}{|c|c|}
\hline Soil Type & Reference \\
\hline Clay (Vicksburg) & 41 \\
\hline Clayey silt & 37 \\
\hline Clay & 38 \\
\hline Clay (VBC) & 39 \\
\hline Clay (VBC) & 39 \\
\hline Clay (Vicksburg) & 40 \\
\hline Lean clay and clay & 25 \\
\hline Lean clay and clay & 25 \\
\hline Lean clay and clay & 25 \\
\hline
\end{tabular}


Table 2 (Cont'd)

Basic Material

Phosphoric acid

Phosphoric acid plus sulfuric acid

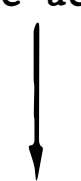

Sulfuric acid

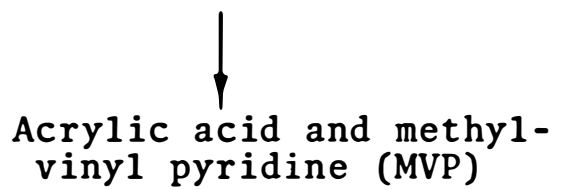

Maleic acid and MVP

Maleic acid, MVP, and methylene-bis acrylamide (MBA)

Acrylic acid and acrylamide

Maleic acid and acrylonitrile
Additives

Soil Type

Sodium fluosilicate octylamine, sulfuric acid, hydrochloric acid, ferric chloride

\section{Aluminum sulfate \\ Ferric chloride}

Ferric chloride

Methylene-bis-acrylamide

(cross-linking agent)

Triacrylyl triazine (crosslinking agent)

MBA
Clay (Vicksburg)

Clayey silt

Clayey silt

Clay (Vicksburg)

Clay

Clay (Vicksburg)

Sandy clay

Sandy clay

Sandy clay

Sandy clay

\section{0}

Sandy clay

34 
Table 2 (Cont'd)

Basic Material

Aspha1t cutback

Asphalt emulsion PR 74

Asphalt, emulsion, straight run

\section{Asphalt fractions}

Emulsified asphalt (SS-1, 66\% asphalt)

MC - 0 asphalt ( $50 \%$ aspha1t)

Modified MC-O asphalt

Straight run asphalt
Additives

Soil Type

Reference

\section{CATEGORY: ASPHALT}

Antistripping additives Water

Pretreatment agents -

Ferric sulfate, alum,

hyamine 1622 , quilon

Various emulsifiers

Phosphorus pentoxide and lauryl amine

Ferric silfate, ferric chloride, aluminum sulfate, alkyl ketenedimer (Aquapel 380), 2-ethylhexylamine, coconut fatty diamine (Duomeen C), soya fatty diamine (Duomeen $S$ ), tallow fatty diamine (Duomeen T), hexamethyl disiloxane, sodium methyl siliconate (SC-50)
Clayey silt

Clayey silt

37

Loess

Loess

24

35

Loess

Clayey silt and buckshot clay 
Table 2 (Cont'd)

Basic Material

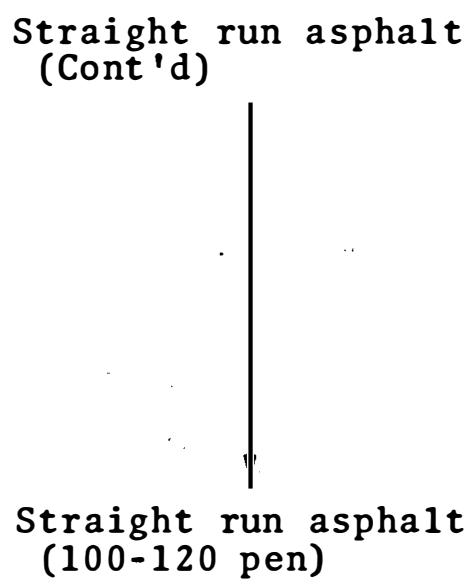

Straight run asphalt (100-200 pen)

Straight run asphalt

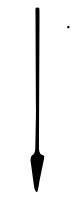

Additives

Soil Type

Reference

Partially polymerized

chloropolysiloxane (SC-87),

dimethylamine ethyl acrylate, quarternized (DV-559),

methylated methylol melamine

(accobond 3913), acrylic

ester resin (acryloid 996)

Armeen 18D (octadecyl amine), Duomeen A - diamine,

ethoduomeen $\mathrm{T}-15$, cationic

agent $A$, nonic 218

Emulsifying agents -

duomeen $\mathrm{T}$, hydrochloric

acid

Solvent - gasoline

Additives - chromic chloride, phosphoric acid

Emulsifying agents -

duomeen $\mathrm{T}$, hydrochloric

acid

Solvent - gasoline

Additive - chromic chloride, phosphoric acid

$$
\begin{aligned}
& \text { Lauryl amine (Armeen 12D) } \\
& \begin{array}{l}
\text { Sand concentrated hydrochloric silt, clayey } \\
\text { silt, sandy clay, } \\
\text { acid emulsion }
\end{array} \\
& \text { Sodium oleate emulsion and clay } \\
&
\end{aligned} \begin{gathered}
\text { Sandy silt, loess, } \\
\text { sandy clay, clayey } \\
\text { silt, and clay }
\end{gathered}
$$


Table 2 (Cont'd)

Basic Material

Teroeas (emulsion)

Vacuum refined asphalt

Hydrated lime

Portland. cement

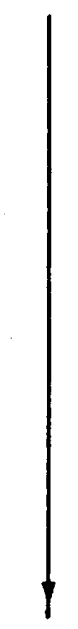

Additives

Armeen 18 acetate, Armeen 18 acetate plus glyceryl monostearate, Armeen 18 acetate plus nonic 218 , ammon ium $\mathrm{N}$-Coco, amino butyrate, ethanolamine oleate, and ammonium oleate

\section{CATEGORY: CEMENT}

Magnesium sulfate and sodium hydroxide

Sodium hydroxide, sodium metasilicate, sodium sulfate

Polyvinyl alcohol, carboxymethyl cellulose, guartec $S F$, guartec $D$

Sodium aluminate

Sodium hydroxide

Sodium hydroxide and sodium metasilicate

Sodium hydroxide, sodium metasilicate, sodium sulfate, octylamine plus sodium hydroxide

Sodium hydroxide, sodium orthosilicate

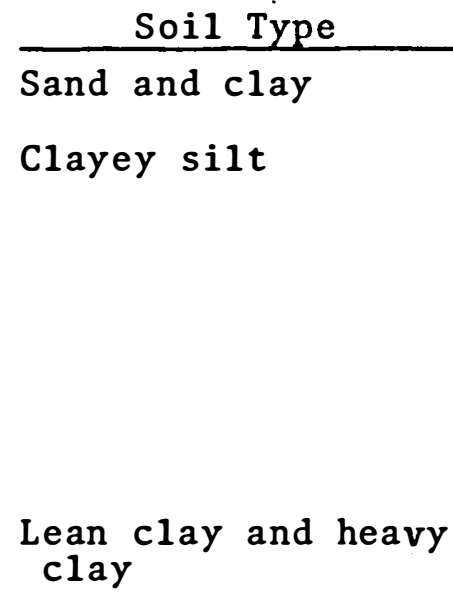

Silt

\section{Lean clay}

Lean clay and clay

Lean clay and clay

Lean clay and clay

Clay (Vicksburg)

Lean clay and clay
38

Reference 5 18

Internal Data (1963), not published

25

25

25

39 
Table 2 (Cont'd)

Basic Material

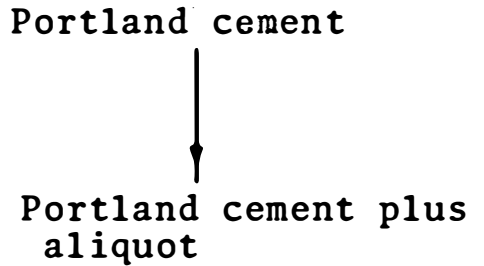

aliquot

Aerospray

Airflex

Ammonium hydroxide

Astro-Soil

Bentonite

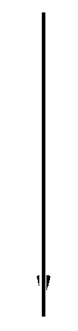

Additives

Sodium sulfate
Sodium sulfate and sodium
orthosilicate

\section{CATEGORY: OTHER}

\begin{tabular}{|c|c|c|}
\hline & Lean clay & $\begin{array}{l}\text { Internal Data } \\
\text { (1960), not } \\
\text { published }\end{array}$ \\
\hline & Sand and silty clay & $\begin{array}{l}\text { Internal Data } \\
(1973) \text {, not } \\
\text { published }\end{array}$ \\
\hline & Clay & 36 \\
\hline$\vdots$ & Clay & $\begin{array}{l}\text { Internal Data } \\
\text { (1971), not } \\
\text { published }\end{array}$ \\
\hline Amides and quaternary salts & Sand & 30 \\
\hline Bentonite gel & Sand & 30 \\
\hline $\begin{array}{l}\text { Bentonite gel plus potassium, } \\
\text { lead, calcium, magnesium, } \\
\text { and aluminum }\end{array}$ & Sand & 30 \\
\hline $\begin{array}{l}\text { Potassium, lead, calcium, } \\
\text { magnesium, and aluminum }\end{array}$ & Sand & 29 \\
\hline
\end{tabular}

$\begin{array}{lc}\text { Soil Type } & \text { Reference } \\ \text { Lean clay and clay } & 25 \\ \text { Lean clay and clay } & 25 \\ \begin{array}{l}\text { Lean clay and heavy } \\ \text { clay }\end{array} & \begin{array}{c}\text { Internal Data } \\ \text { (1961), not } \\ \text { published }\end{array}\end{array}$


Table 2 (Cont'd)

Basic Material

Bindarene flour (1ignin)

Bindarene flour

Calcium $\left(\mathrm{CA}(\mathrm{OH})_{2}\right)$ and magnesium hydroxide $\left(\mathrm{Mg}(\mathrm{OH})_{2}\right)$

Calcium carbide

Calcium hydroxide $\mathrm{Ca}(\mathrm{OH})_{2}$

Calcium hydroxide plus magnesium sulfate

Calcium hydroxide plus sodium hydroxide

Calcium oxide
Additives

Potassium bichromate, aluminum

Potassium bichromate, calcium oxide, magnesium sulfate, borax, portland cement, barium chloride, Monsanto resin CRD 197 , sodium phosphate, sodium formate, sodium aluminate, sodium silicate, and sodium hydroxide

Sodium bichromate, potassium bichromate

$\mathrm{MgSO}_{4}$

Lean clay

Lean clay and heavy
clay

Sodium hydroxide, magnesium sulfate, potassium sulfate

\section{Clay (Vicksburg)}

Lean clay and clay

Lean clay and clay

Magnesium sulfate and Dustrol Heavy clay
Reference 5

5

5

Internal Data (1961), not published

Internal Data (1956-57), not published

40

25

25

Internal Data (1960), not published 
Table 2 (Cont'd)

Basic Material

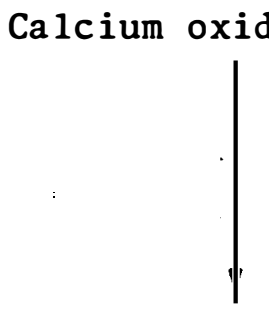

Calcium oxide plus magnesium sulfate

Calcium oxide plus sodium hydroxide

Carboxy methyl cellulose (CMC)
Additives

Magnesium sulfate, sodium metasilicate, zinc sulfate, nickel sulfate

\section{Polyvinyl alcohol plus} carboxymethyl cellulose

Alkyl dimethyl benzyl ammonium chloride

Amine $D$ acetate

n-octylamine

Octadecyl amine acetate

Octadecyl amine

Sodium orthosilicate
Soil Type

Shale (Suburua soft)

Lean clay and heavy clay

Lean clay and clay

Lean clay and clay

Lean clay and clay

Lean clay and clay

Lean clay and clay

Lean clay and clay

Lean clay and clay

Lean clay and clay

Sand, clay, and sandy clay

Lean clay and heavy clay
Reference

41

Internal Data

(1963), not

published

25

25

25

25

25

25

25

25

5

Internal Data

(1956-57)

not published 
Table 2 (Cont'd)

Basic Material

Casein 141-V

Casein 1221V

Casein plus hydrated

lime plus ferric oxide

(ratio - 55:13:3)

Casein glue

Chrome lignin

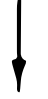

Cla-Pak

Cla-Set

Compact

Daimond Siroc
Additives

Lean clay

Lean clay

Lean clay

Lean clay

Silt

Clay

Lean clay and heavy clay

Lean clay and heavy clay

Lean clay

Lean clay
Reference

Internal Data (1960), not published

Internal Data (1960), not published

Internal Data (1958), not published

Internal Data (1960), not published

22

36

Internal Data (1974), not published

Internal Data (1974), not published

Internal Data (1972), not published

Internal Data (1964), not published 
Table 2 (Cont'd)

\begin{tabular}{|c|c|c|c|}
\hline Basic Material & Additives & Soil Type & Reference \\
\hline Dustmaster & & Lean clay & $\begin{array}{l}\text { Internal Data } \\
\text { (1972), not } \\
\text { published }\end{array}$ \\
\hline Dustmaster. "C" & & Lean clay & $\begin{array}{l}\text { Internal Data } \\
\text { (1972), not } \\
\text { published }\end{array}$ \\
\hline Dustmaster "WR" & & Lean clay & $\begin{array}{l}\text { Internal Data } \\
\text { (1972), not } \\
\text { published }\end{array}$ \\
\hline Dustrol (road oil) & & Loess & 24 \\
\hline Ecology Control M-Binder & & Lean clay & $\begin{array}{l}\text { Internal Data } \\
\text { (1973), not } \\
\text { published }\end{array}$ \\
\hline Erode $-\mathrm{X}$ & & Lean clay & $\begin{array}{l}\text { Internal Data } \\
\text { (1972), not } \\
\text { published }\end{array}$ \\
\hline Ferrous lignosulphonate & & Silty sand & 36 \\
\hline Florok & & Clay & $\begin{array}{l}\text { Internal Data } \\
\text { (1965), not } \\
\text { published }\end{array}$ \\
\hline Formula 125 & & $\begin{array}{l}\text { Lean clay and heavy } \\
\text { clay }\end{array}$ & $\begin{array}{l}\text { Internal Data } \\
\text { (1974), not } \\
\text { published }\end{array}$ \\
\hline Formula 2221 & & $\begin{array}{l}\text { Lean clay and heavy } \\
\text { clay }\end{array}$ & $\begin{array}{l}\text { Internal Data } \\
\text { (1961), not } \\
\text { published }\end{array}$ \\
\hline
\end{tabular}


Table 2 (Cont'd)

Basic Material

\section{Genaqua erosion control} latex 169

\section{Guartec D}

Guartec SF

Huls 801

Iron polyphosphate

Kel-Pak

Laurylamine

Lignin

Lignin (unoxidized)

Magnesium oxychloride
Additives

Soil Type

Reference

Lean clay

Lean clay and heavy clay

Lean clay and heavy clay

Lean clay

Sodium tetraphosphate, ferrous chloride, ferric chloride

andy clay

Lean clay and heavy clay

Lean clay and clay

Clay

Ferric chloride, phosphor us pentoxide, aluminum sulfate, sodium peroxide, stannic chloride, sodium chlorate

Sand, sandy clay, and clay

Sandy clay
Internal Data (1972), not published

Internal Data (1963), not published

Internal Data (1963), not published

Internal Data (1972), not published

34

Internal Data (1974), not published 
Basic Material

Mortuary hardening compound

n-octylamine

Octadecyl amine

Orthorhombic phosphoric anhydride

Orzan -50

Paczyme

Pectosol

Pen-E-Pac

Pen-E-Pac plus asphalt

Phosphate rock

Plasmofalt

Polyvinyl alcohol (PVA) and CMC
Additives

Soil Type

Lean clay

Sodium fluosilicate, octylamine, phosphoric acid, ferric chloride

Lean clay

Clayey silt

Clay

Lean clay

Lean clay

Sulfuric acid

Lean clay and heavy clay

Sandy clay

Loess, heavy clay, and sandy clay
Reference

Internal Data (1959), not published 25 25 40

Internal Data (1972), not published

27

5

Internal Data (1970), not given

Internal Data (1970), not published

18

31

Internal Data (1956), not published 
Table 2 (Cont'd)

\section{Basic Material}

Sodium bentonite

Sodium pectate

Soil Master

Speed crete

Styrene

Sylon (alkoxy amine silane)

Terra-Krete

Verdyol Super

Westco D-1
Additives

Calcium chloride, lead acetate

Sodium phosphate, sodium bichromate

Emulsifying agents methyl-vinyl pyridine, polymethyl-vinyl pyridine Catalyst - cyclohexanone peroxide Accelerator - cobaltnaphthenate

\begin{tabular}{|c|c|}
\hline Soil Type & Reference \\
\hline Sandy clay & 30 \\
\hline Not given & 5 \\
\hline Clay & $\begin{array}{l}\text { Internal Data } \\
\text { (1971), not } \\
\text { given }\end{array}$ \\
\hline Lean clay & $\begin{array}{l}\text { Internal Data } \\
\text { (1959), not } \\
\text { published }\end{array}$ \\
\hline Sandy clay & 35 \\
\hline $\begin{array}{l}\text { Sand, sandy clay, } \\
\text { and clay }\end{array}$ & 5 \\
\hline $\begin{array}{l}\text { Lean clay, heavy } \\
\text { clay, and sand }\end{array}$ & $\begin{array}{l}\text { Internal Data } \\
\text { (1973), not } \\
\text { published }\end{array}$ \\
\hline Lean clay & $\begin{array}{l}\text { Internal Data } \\
\text { (1973), not } \\
\text { published }\end{array}$ \\
\hline
\end{tabular}

Lean clay 
Table 2 (Cont'd)

Basic Material

\section{Quicklime}

Quilon (stearate chromic chloride)

RD-4516

$\mathrm{RD}-4518$

Reynolds Road Packer (1 part Road Packer to 1000 parts water by volume)

RE्षI Moldit (418-2)

R\&्GI Moldit (419-2)

Roadseal \#17

SA-1

SC -100

Additives

Soil Type

Lean clay

Clay

Lean clay

Lean clay

Lean clay

Lean clay and heavy clay

Lean clay and heavy clay

Lean clay

Silty clay

Loess and heavy clay
Reference

13

5

Internal Data (1974), not published

Internal Data (1974), not published

Internal Data (1961), not published

Internal Data (1957), not published

Internal Data (1957), not published

Internal Data (1972), not published

28

Internal Data (1957), not published 
Table 2 (Cont'd)

Basic Material

Westco D-2

$X B-2386$

Calcium phosphate plus sulfuric acid

Methyl-vinyl pyridine (MVP), methylene-bisacrylamide (MBA), and benzene phosphoric acid

MVP and sulfuric acid

MVP, trisacryl, and benzene phosphoric acid

Phosphate rock (71.48

by weight $\mathrm{CA}_{3}\left(\mathrm{PO}_{4}\right)_{2}$ and $3 \&$ fluorine) plus sulfuric acid

Phosphate rock and sulfuric acid
Additives

Soil Type

Reference

Lean clay

Lean clay and heavy clay

\section{CATEGORY: OTHER/ACID}

$\begin{array}{ll} & \text { Clayey silt } \\ \text { Water } & \text { Sandy clay } \\ \text { Catalyst - ammonium } & \\ \text { persulfate } & \\ \text { Activator - sodium } & \\ \text { thiosulfate } & \\ \text { Water } & \text { Sandy clay } \\ \text { Catalyst - ammonium } & \\ \text { persulfate } & \\ \text { Activator - sodium } & \\ \text { thiosulfate } & \\ \text { Water } & \text { Sandy clay } \\ \text { Activator - sodium } & \\ \text { thiosulfate } & \\ \text { Catalys - ammonium } & \\ \text { persulfate } & \\ \text { Octylamine and ferric } & \\ \text { chloride } & \text { Clay (Vicksburg) }\end{array}$


Table 2 (Cont'd)

Basic Material

Phosphate rock plus sulfuric acid

Acrylonitrile

American resinous emulsion $382-37 \mathrm{C}$

\section{American resinous} emulsion 1073-18H

American resinous emulsion 1450-15B

Aniline-furfural
Additives

Additive - sodium fluosilicate Waterproofing agents Octylamine and Armeen 8

\section{CATEGORY : RESIN}

Emulsifying agents -

Methyl-vinyl pyridine and

polymethyl-vinyl pyridine

Catalyst - potassium sulfate

Accelerator - sodium

bisulfite

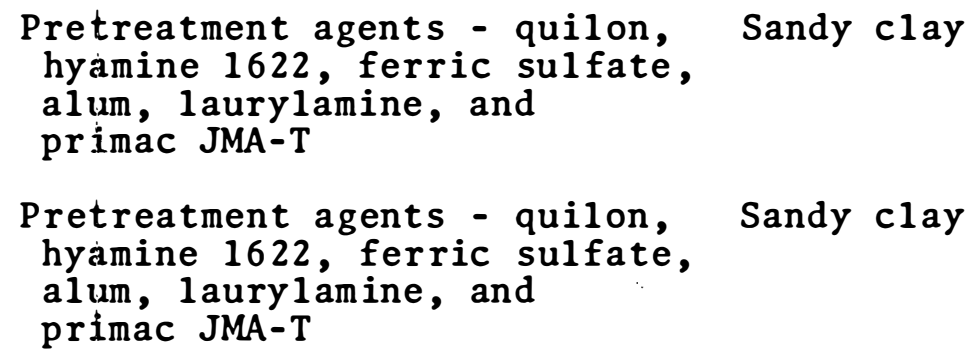

Pretreatment agents - quilon, hyamine 1622 , ferric sulfate, alum, laurylamine, and primac JMA-T

Pretreatment agents - quilon, Sandy clay hyamine 1622 , ferric sulfate, alum, laurylamine, and primac JMA-T

Phthalic acid (catalyst) 
Table 2 (Cont'd)

Basic Material

Aniline-furfural

Arboneeld B

Arlon 110

Arlon 310 (air-dry

alkyd)

Arlon 363

Arlon 580 (air-dry a $1 \mathrm{kyd}$ )

Aroplaz 832

Aroplaz 6008

Aroplaz 6065

Ashland experimental emulsion

Barium acrylate

Butyl methacrylate

Calcium acrylate
Additives

Solox

Sand and clay

5

Sand, clay, and sandy clay

Sand

57

Sand

57

Sand

57

Solvent - water

Sand

57

Solvent - JP-4

Sand

Solvent - JP-4

Sand

57

Solvent - JP-4

Sand

57

Solvent - water

Sand and clay

57

Water

AP/ST - catalyst/activator

Sandy clay

(arnmonium persulfate-

sodium thiosulfate)

Polyvinyl alcohol (emulsifier)

Sandy clay

36

Dimethyl aniline (catalyst)

Benzoyl peroxide (accelerator).

Zinc sulfate and sodium

Sandy clay

31

57

7

7

7
sulfate

clay 
Table 2 (Cont'd)

Basic Material

Calcium acrylate

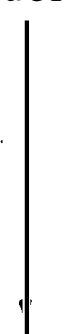

Calcium acrylate and ammonium acrylate

Calcium acrylate and
calcium methacrylate

Calcium acrylate and ethylene glycol

diacrylate

Calcium acrylate and lithium acrylate
Additives

Catalyst - aluminum

persulfate

Catalyst activator - sodium thiosulfate

Ammonium persulfate-sodium thiosulfate system

Ferrous ions (as activator for above system)

Water

AP/ST - catalyst/activator

(ammonium persulfatesodium thiosulfate)

Water

AP/ST - catalyst/activator (ainmonium persulfate and sodium thiosulfate)

Water

AP/ST - catalyst/activator (ammonium persulfate and sodium thiosulfate) (See comments on page A227)

Water

AP/ST - catalyst/activator (ammonium persulfatesodium thiosulfate)

\section{Soil Type}

Sandy clay

30

Sandy clay

Sandy clay

Sandy clay

Sandy clay

Sandy clay

31 
Table 2 (Cont'd)

Basic Material

\section{Calcium acrylate and} manganese acrylate

Calcium acrylate and magnesium acrylate

Calcium acrylate and methylene-bisacrylamide

Calcium acrylate and monoamine acrylates

Calcium acrylate and $\mathrm{N}$-methylolacrylamide

Calcium acrylate and organic nonionic monomers

Calcium acrylate and . precondensed $\mathrm{N}$ methylolacrylamide

Calcium acrylate and nickel acrylate

Calcium acrylate and potassium acrylate.
Additives

Water

AP/ST - catalyst/activator

(ammonium persulfate-

sodium thiosulfate)

Water

AP/ST - catalyst/activator

(ammonium persulfate-

sodium thiosulfate)

"1
Sandy clay

Sandy clay

31

Sandy silt

Sandy clay

31

Sandy soil

31 
Table 2 (Cont'd)

Basic Material

Calcium acrylate and sodium acrylate

Calcium acrylate and $z$ inc acrylate

Calcium acrylate, zinc, and sodium acrylate

Calcium methacrylate

Chem Rez 200

Coherex

Creosote bush extract

DC 804 silicone resin

DC 2103 silicone resin

Dimethyl aminoethyl acrylate

DRC resin
Additives

Water

AP/ST - catalyst/activator

(amnonium persulfate-

sodium thiosulfate)

"1

Sandy clay

Sandy clay

Sandy clay

31

persulfate, t-butyl

hydroperoxide, hydrogen

peroxide, and urea peroxide

\section{Solvent - solox}

Solvent - water

See Reference 30

See Reference 30

Sand

Sand

Clay

Lean clay

Reference

31

Sand

Sand and clay

Lean clay

Internal Data (1959), not published

30

30

36

Catalyst - 2 parts of cobalt napthenate to 1 part lead napthenate
Internal Data
(1960), not published


Table 2 (Cont'd)

Basic Material

Emlon E-200

EP 8908-23

EP 8908-122

EP 8908-129

Epiphen ER 823

Epon VI

Epon 562

Epon 828

Epon 834
Additives

Solvent - water

Curing agents - diethylene triamine, diethylaminomethyl phenol, combination of above curing agents

Curing agents - Agent A (amine), diethylenetriamine water

Acetone (solvent); curing agent and waterproofers for treated samples (See

Reference 37)

Curing agent diethylenetriamine Water

Curing agent and hydroxides

Xylene

Curing agent - DMP-30 (tri

dimethylaminomethyl phenol)
Soil Type

Sand and clay

Sand

Sand

Sand

Sandy clay

Reference 
Table 2 (Cont'd)

Basic Material

Epon RL 1062

Epon Rn 34

$15 x P F$ gelatin

Hexamethylolmelamine

Isomerized glyceryl ester of resin

Laminac 4116 (a1ky1 styrene resin)

Laminac 4134

Manganese acrylate

Magnesium acrylate

Melamine
Additives

Curing agents -

Diethylenetriamine,

tetraethylenepentamine, water

Catalyst - chromium sulfate and formaldehyde

Solvent - water

Catalyst "AC"

Hydrochloric acid

Water

AP/ST - catalyst/activator

(ammonium persulfate-

sodium thiosulfate)
Sand and clay

5

Sand

Sand

30

Loess

24

Sand and clay

5

Sand, sandy clay, and clay

Sandy clay

32

Sand

Sandy clay

32

29 
Basic Material

Methoxy ethyl acrylate

Methyl acrylate and calcium acrylate

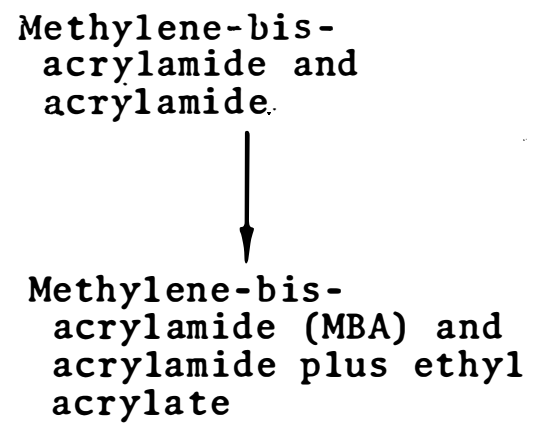

MBA and acrylic acid MBA and sodium
acrylate
Additives

Emulsifiers - polyvinyl alcohol and octadecyl trimethyl ammonium chloride

Catalyst - dimethyl aniline

Accelerator - benzoyl peroxide

Emulsifier - polyvinyl alcohol Sandy clay

Soil Type

Reference

Sandy clay

Catalyst - potassium

persulfate

Accelerator - sodium

bisulfite

Water

AP/ST - catalyst/activator

(ammonium persulfate-

sodium thiosulate)

Glyoxal (reactant)

Ethanol plus water plus ethyl acrylate

AP/ST - catalyst/activator (ammonium persulfatesodium thiosulfate)
Dispersants - Quadrafos and aerosol AY
Pretreatments - Volan, hyamine 1622 , aluminum sulfate

\section{Water}

AP/ST - catalyst/activator (ammonium persulfatesodium thiosulfate)
Sandy clay

Sandy clay

Sandy clay

Sandy clay

Sandy clay 
Table' 2 (Cont'd)

\begin{tabular}{|c|c|c|c|}
\hline Basic Material & Additives & Soil Type & Reference \\
\hline Natural shellac & & Sand & $30^{\circ}$ \\
\hline n-methylolacrylamine & See Reference 31 . & Sandy clay & 31 \\
\hline Nickel acrylate & $\begin{array}{l}\text { Water } \\
\text { AP/ST - catalyst/activator } \\
\text { (ammonium persulfate- } \\
\text { sodium thiosulfate) }\end{array}$ & Sandy clay & 32 \\
\hline $\begin{array}{c}\text { Parez } 620 \text { (cationic } \\
\text { urea-formaldehyde) }\end{array}$ & & Sand and clay & 5 \\
\hline Perma-Soil & & Lean clay & $\begin{array}{l}\text { Internal Data } \\
\text { (1972), not } \\
\text { published }\end{array}$ \\
\hline Petroset & & Lean clay & $\begin{array}{l}\text { Internal Data } \\
\text { (1974), not } \\
\text { published }\end{array}$ \\
\hline Polycalcium acrylate & $\begin{array}{l}\text { Sodium thiosulfate and } \\
\text { ammonium persulfate }\end{array}$ & Clay (Kaolin) & 30 \\
\hline \multirow[t]{2}{*}{ Polylite 8000} & See Reference 35 & Sandy clay & 35 \\
\hline & $\begin{array}{l}\text { Arquad } 12 \text { and Armeen 12D (EA) } \\
\text { Acrylamide (monomer) } \\
\text { Potassium persulfate (catalyst) } \\
\text { Sodium bisulfite (accelerator) } \\
\text { Water added on soil } \\
\text { EA - emulsifying agent }\end{array}$ & Sandy clay & 36 \\
\hline
\end{tabular}


Tab́le 2 (Cont'd)

Basic Material

\section{Polylite 8000}

Polylite 8009

$$
\mid
$$

Polylite 8009 and 8120

\section{Polymer emulsions (see comments on page A301)}

Polyvinyl acetate

Polyvinyl acetate and acrylamide

Resimene 815

Resinox L10060
Additives

See Reference 36

See Reference 35

Emulsifying agent methyl-vinyl

pyridine, polymethyl-vinyl

pyridine (See Reference 35

for various catalysts and accelerators used)

\section{Emulsifying agent - A12-A12D}

Catalyst - MEKP

Accelerator - CN

Water

Ammonium persulfate, sodium thiosulfate, and water (on soil)

Catalyst - "AC"

1\% hydrochloric acid
Sandy clay

Sandy clay

Soil Type

Sandy clay

Sandy clay

Sand and sandy clay

Sandy clay

Sandy clay

Sand

Sand
35

35

35

31

36

36

30

36

6 
Table 2 (Cont'd)

Basic Material

Resinox 407

Resinox 426

Resorsabond

Resorsabond R-11

Resorsabond R-12

Shellac

Strontium acrylate

Styrene emulsion

Trimethy lolmelamine

Urea-melamineformaldehyde ( $31 \%$ nonaqueous solids)

(48.5\% nonaqueous solids)

(48.5\% nonaqueous solids)
Additives

Resinox 408 hardener

Soil Type

Water

AP/ST - catalyst-activator

(ammonium persulfate-

sodium thiosulfate)

Various solvents used Toluene, benzene, and methylene dichloride

Catalyst - "AC"

$1 \%$ hydrochloric acid

Phthalic salicylic and maleic acids (both catalysts)

See Reference 30

Butex, 4 C-BL, admixtures of both, catalyzed with phthalic acid
Sandy clay

Sand

Sand

Sand

Sand

Sand

Sandy clay

Sand

Reference

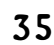

29

29

30

30

30

32

N/A 
Basic Material

\section{Urea-melamine - formaldehyde ( $48 \%$ nonaqueous solids)}

(48.5\% nonaqueous solids)

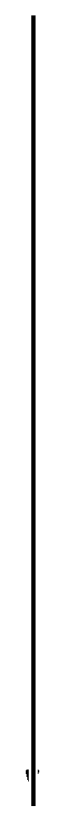

Zinc acrylate

Zinc acrylate

Zinc acrylate and ammonium acrylate
Additives

Catalysts - chloroacetic, maleic, succinic, tartaric, terephthalic acids, and potassium acid phthalate

Hydrotropic agents (see

Catalyst - phthalic acid

Phthalic acid ( $\mathrm{pH} 3.9)$

(cure time varied)

Phthalic acid (pH 3.9)

Polyvinyl alcohol, Elvanol 50-42A, Elvanol 50-42B, Elvanol 72-51A, and Elvanol 20-105A

Polyvinyl acetate modification

See Reference 30 for surface active agents

Water

Sandy clay

Lean clay and heavy clay

Sandy clay

Reference sodium thiosulfate) 
Table 2 (Cont'd)

Basic Material

Zinc acrylate and potassium acrylate

Zinc acrylate and lithium acrylate

Zinc and magnesium acrylate

\section{Zinc-magnesium} acrylate

\section{Zinc acrylate and} sodium acrylate

Butyl methacrylate, acrylic acid, and calcium acrylate

Calcium acrylate and acrylic acid
Additives

Water

AP/ST - catalyst/activator

(ammonium persulfate-

sodium thiosulfate)

"

Water

Water

AP/ST - catalyst/activator

(ammonium persulfate-

sodium thiosulfate)

\section{CATEGORY : RESIN/ACID}

Emulsifier - polyvinyl

alcohol; catalyst -

potassium persulfate;

accelerator - sodium

bisulfite
Sandy clay

Lean clay and heavy clay

Soil Type

Sandy clay

Sandy clay

31

32

Reference

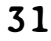

Internal Data

(1956-57)

not published

Sandy clay

31

36

Sandy clay
31 
Basic Material

\section{Calcium acrylate, zinc acrylate, plus acrylic acid \\ Ethyl methacrylate, acrylic acid, and calcium acrylate}

\section{Magnesium acrylate plus acrylic acid
Methoxy ethyl acrylate, acrylonitrile, acrylic acid

MBA and acrylamide plus acrylic acid

MBA, acrylamide, and acrylic acid

MBA and acrylic acid
Water

AP/ST - catalyst-activator

(ammonium persulfatesodium thiosulfate)

Emulsifier - polyvinyl alcohol; catalyst -

potassium persulfate; accelerator - sodium bisulfite

Water

Emulsifier - polyvinyl alcohol; catalyst potassium persulfate; accelerator - sodium bisulfite

Water plus acrylic acid AP/ST - catalyst/activator (ammonium persulfatesodium thiosulfate)

Complexing agents chromium chloride and aluminum sulfate

Water

Catalyst - ammonium

persulfate and hydroxylamine hydrochloride (activator)
Sandy clay

Sandy clay

Sandy clay

Sandy clay 
Table 2 (Cont'd)

Basic Material

MBA and acrylic acid

MBA and acrylic acid

MBA and acrylic acid

$$
\mid
$$

MBA, acrylic acid, and acrylonitrile

MBA, acrylic acid, $\mathrm{N}, \mathrm{n}$ dimethylacrylamide

MBA, acrylic acid, and methoxyethyl acrylate

MBA, acrylic acid, and methyl vinyl ketone

MBA and methacrylic acid
Additives

\section{Water}

Ammonium persulfate-

hydroxylamine hydrochloride (catalyst/activator)

Dimethylformamide (reactant) Sandy clay

Dispersants - quadrafos

(sodium tetraphosphate), aerosol $\mathrm{AY}$, and Triton X-100

Ethylene glycol (reactant) Sandy clay

Polyvinyl alcohol (esterifica- Sandy clay tion agent); ethylene glycol (secondary reagent)

Water

Water

$\mathrm{AP} / \mathrm{ST}$ - catalyst/activator

(ammonium persulfate-

sodium thiosulfate)
33

Sandy clay

Sandy clay

Sandy clay

Sandy clay 
Basic Material

Calcium acrylate and
ethylene diamine
Calcium acrylate and
hexamethylene diamine
Melamine formaldehyde
and acrylamide
Zinc acrylate plus
acrylic acid
Calcium acrylate and
acrylate salts (see
comments on page
A359)
Calcium chloride
Alkyl dimethyl,
benzyl ammonium
chloride.

Additives

Soil Type

Reference

\section{CATEGORY : RES IN/OTHER}

\section{Water \\ AP/ST - catalyst/activator (ammonium persulfate and sodium thiosulfate)}

11

$$
\begin{aligned}
& \text { Catalyst - ammonium } \\
& \text { persulfate } \\
& \text { Activator - sodium } \\
& \text { thiosulfate }
\end{aligned}
$$

Water

Water

AP/ST - catalyst/activator

(ammonium persulfatesodium thiosulfate)

\section{CATEGORY: SALT}

Sandy clay

Sandy clay

Sandy

Sandy clay

Sandy clay

Silty sand and clay

Lean clay and clay

Silty sand

Loess 
Table 2 (Cont'd)

Basic Material

Dialkyl dimethyl ammonium chloride

\section{Ferric chloride}

Lithium chloride

Sodium chloride

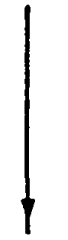

Ethyl silicate

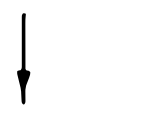

Ludox (colloidal silica)

Magnesium orthosilicate

Sodium

metasilicate

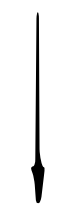

Additives

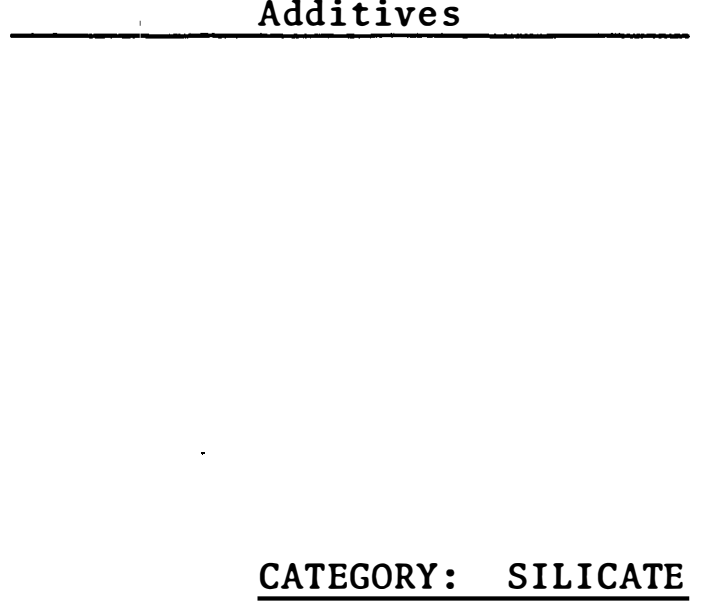

\section{Hydrochloride acid}

Sand

Soil Type

Silt

Silty sand and clay

Clay

Silty sand

Lean clay and heavy clay

Hydrogen chloride

Sand

Sand, clay, and sandy clay

Magnesium oxide

Lean and heavy clay

Lean clay and clay

Magnesium carbonate

Magnesium oxide
25

23
36
36
36

Internal Data (1961), not published

29

30

5

18

18

18

18 
Table 2 (Cont'd)

Basic Material

Sodium orthosilicate

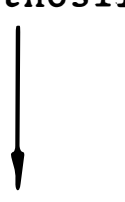

Sodium silicate

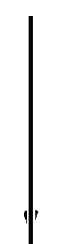

Sodium silicate ( 328 solution)

Sodium silicate solution (in aqueous solution of $38 \%$. concentration of sodium silicate) $\left(\mathrm{Na}_{2} \mathrm{O} \cdot \mathrm{SiO}_{2}\right)$

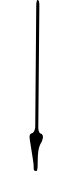

Sodium metasilicate plus magnesium carbonate
Additives

Magnesium carbonate

Lead, calcium, aluminum, magnesium, nickel, zinc, and zirconium

Calcium, lead, aluminum, magnesium, nickel, and $z$ inc

Calcium chloride, lead acetate

Magnesium carbonate

Sandy clay

Lean and heavy clay

Lean and heavy clay

Magnesium oxide

Soil Type

Lean clay and clay

Lean and heavy clay

Lean and heavy clay

S and

Sand
CATEGORY: SILICATE/OTHER

Lean clay and clay

\section{0}


Table 2 (Cont'd)

Basic Material

Sodium orthosilicate

plus magnesium carbonate

Sodium orthosilicate

plus magnesium oxide

Sodium silicate plus

basic magnesium

carbonate

Sodium silicate solution plus magnesium

carbonate

Sodium silicate solution plus magnesium oxide
Additives

Soil Type

Reference

Lean clay and clay

25

Lean clay and clay

25

Clay (Vicksburg)

40

Lean clay and clay

25

Lean clay and clay

25 
Table 3

MOST EFFECTIVE MATERIALS FOR EACH SOIL TYPE

Soil Type

Silt

Loess

Clay

Sand
Unconfined Compressive Strengths, psi

\begin{tabular}{|c|c|c|c|c|}
\hline Untreated & Cement & Lime & Asphalt & Other Best Material \\
\hline 20 & $80-280$ & $230-860$ & 225 & Sodium silicate - 650 \\
\hline 20 & 100 & $160-970$ & -- & Powders $A$ and $B-389$ \\
\hline 20 & $76-300$ & $100-340$ & $104-389$ & Calcium oxide - 315 \\
\hline 20 & $150-425$ & -- & - & Aropol 7110 - 1170-1890 \\
\hline
\end{tabular}


1. The information contained in this appendix covers the materials subjected to investigation and tests. These materials are grouped by ćategory (material categories listed below), secondary materials, and date of report. Information listed as "not given" was not listed in the referenced report and not available from other sources at WES. When the "rate of material" is listed as "varied," several different rates were used in the testing program. The "mixing capability" is listed as "good" when no reference to this item is given in the reports. Definitions of terms and tests used in this appendix are presented below:

a. MIT. Massachusetts Institute of Technology.

b. WES. U. S. Army Engineer Waterways Experiment Station.

c. Effectiveness categories. Excellent, moderate, slight, none, or detrimental.

d. Material categories. Resin, asphalt, cement, salt, lime, acetate, acid, silicate, or other ("other" includes materials not in one of the given categories or material for which the proper category was not known).

e. Mixing capabilities. Excellent, good, difficult, or impossible.

f. Tast types and categories of stabilization:

(1) MIT unconfined compression test (Reference 29). Test specimens are prepared in cylindrical molds about $1-1 / 2$ in.* in diameter and about 3 in. tall. The specimen is put in the mold and then tamped by means of a light piston about 1 in. in diameter. No standard compaction procedure is used, but it is believed that all specimens receive similar compaction. This light tamping is not believed to have much effect on the compressive strength of the speci- . men except for the effect caused by air pockets being eliminated. The strength of the specimen is determined in simple compression; this method is a rapid, reliable method of determining the shearing strength. For indication of absorption or capillary rise of water, the specimen is immersed in water either

* A table of factors for converting U. S. customary units of measurement to metric (SI) units is presented on page 4. 
completely or to a depth of about $1 \mathrm{~cm}$. The specimen is observed visually and then subjected to unconfined compression tests when wet and when redried.

(2) MIT tensile test (Reference 30). Soil specimens are prepared with the chemical material. These specimens are 3 in. long with a l-in.-long by 1/2-in.-wide portion at the mid-section. The applied load is measured by a proving ring.

(3) MIT compression test (Reference 35). The Harvard Miniature Compaction Apparatus is used in specimen preparation. The dimensions of the mold are $2.82 \mathrm{in}$. in length and 1.312 in. in diameter. The specimens are prepared in three layers and compacted by 25 tamps per layer of a 40-lb load.

(4) Category 1 stabilization* (References 13 and 37). This is obtained if the chemical additive can increase, within a 2-hr limit, the strength of the soil from a cone index of 20 (equivalent to $1 \mathrm{CBR}$ or less) to 120 (equivalent to a minimum CBR of 4), with this latter value deemed adequate for light traffic.

(5) Category 2 stabilization** (References 14 and 16). This condition occurs when a stabilizer is capable of increasing the compressive strength of the soil from about 25 psi ( $4 \mathrm{CBR}$ ) to about $100 \mathrm{psi}(20 \mathrm{CBR}$ ) or greater after $24 \mathrm{hr}$ curing without benefit of drying.

(6) Test procedures for unconfined compression tests for soil stabilizers and waterproofers; permeation method (Reference 24):

(a) Untreated soil and treated soil are compacted in a Harvard miniature mold ( 1.312 in. in diameter by $2.82 \mathrm{in}$. long). Compaction is achieved by applying 20 tamps with a 40-1b spring to each of five equal layers. The specimens are then extruded from the mold and permitted to cure under ambient laboratory conditions for a period of at least 4 days.

(b) The compacted, air-dried, treated specimens are placed in a rubber membrane, and water is permitted to enter the top and flow downward through it. Duplicate untreated specimens are also subjected to water. After 4 days of permeation, the specimens are subjected to unconfined compression tests.

\footnotetext{
* Also referred to as "emergency requirements."

** Also referred to as "routine requirements."
} 
(7) Test procedures for unconfined compression tests for soil stabilizers and waterproofers; capillary method (Reference 51):

(a) Untreated soil and treated soil are compacted in a Harvard miniature mold ( 1.312 in. in diameter by 2.82 in. long). Compaction is achieved by applying 20 tamps with a 40-lb spring to each of five equal layers. The specimens are then extruded from the mold and permitted to cure under ambient laboratory conditions for a period of at least 4 days.

(b) The air-dried specimens are then put in a membrane that is open at both ends and placed in an upright position on a 3/8-in.-thick porous stone in an evaporating dish. Water is placed in the bottom of the dish, the level of the water being maintained approximately $1 / 8$ in. below the bottom of the specimens for a period of 4 days. This 4-day period is considered to be a cycle. After the specified number of cycles has been completed, unconfined compression tests are then conducted on the specimens.

(8) Emergency requirements. See Category I stabilization. .

(9) Routine requirements. See Category 2 stabilization.

(10) Traffic tests. Details are given in the referenced reports. 
Category: Acid 

Category*
Acid
Basic Material
Rate of Material
Cost
Phosphoric acid
$\left(\mathrm{H}_{3} \mathrm{PO}_{4}\right)$
$0.5,1.0,1.5,2.0,3.0 \% \quad$ Not given
Secondary Material
Sodium fluosilicate
$0.5 \%$
Not given
Mixing
Material Form*
Type of Soil Treated
Capability
Liquid
Clayey silt
Good

\begin{tabular}{|c|c|c|c|c|}
\hline Type of Test & $\begin{array}{l}\text { Purpose } \\
\text { Material } \\
\end{array}$ & & $\begin{array}{l}\text { Effective } \\
\text { Strength } \\
\text { Increase } \\
\end{array}$ & Effectiveness \\
\hline $\begin{array}{l}\text { Unconfined } \\
\text { compression }\end{array}$ & Stabilizer & & See comments & Excellent \\
\hline $\begin{array}{c}\text { Total Material } \\
\text { Per Cu Ft } \\
\text { of Treated So }\end{array}$ & $\begin{array}{l}\text { Cost } \\
\text { il }\end{array}$ & Test & Agency & Test Report \\
\hline Not given & & MIT & & Reference 40 \\
\hline
\end{tabular}

Samples treated with $0.5 \%$ sodium fluosilicate and various rates of phosphoric acid not compared to untreated samples. Tests were conducted after.a 24 hours water immersion.

\begin{tabular}{|c|c|c|}
\hline . & $\begin{array}{l}3^{P^{2}} 4 \\
(\%) \\
\end{array}$ & Strength (psi) \\
\hline (Continued on next & $\begin{array}{l}0.5 \\
1.0 \\
1.5 \\
2.0 \\
3.80)\end{array}$ & $\begin{array}{l}85 \\
- \\
170 \\
325 \\
630\end{array}$ \\
\hline
\end{tabular}

* Basic material 
Effectiveness: As seen from the data above, once the amount of $\mathrm{H}_{3} \mathrm{PO}_{4}$ reaches 1.5 percent, the strength of the samples is very good and with a small amount of increase in the acid, a significant increase in strength is achieved. 
Category*

Acid

Basic Material.

Rate of Material

Phosphoric acid $\left(\mathrm{H}_{3} \mathrm{PO}_{4}\right)$

2 and $3 \%$

Cost

Not given

Secondary Material

See comments

Material Form*

Liquid
Type of Soil Treated

Lean clay

Heavy clay
Mixing

Capability

Good

Good

\begin{tabular}{lllll} 
Type of Test & $\begin{array}{l}\text { Purpose of } \\
\text { Material }\end{array}$ & $\begin{array}{l}\text { Effective } \\
\text { Strength } \\
\text { Increase }\end{array}$ & Effectiveness \\
\cline { 1 - 2 } $\begin{array}{c}\text { Unconfined } \\
\text { compression }\end{array}$ & Stabilizer & See comments & Excellent
\end{tabular}

Total Material Cost Per Cu Ft of Treated Soil

Test Agency

WES
Test Report

Reference 18

Comments:

Samples were molded in a Harvard Miniature Compaction Apparatus in five layers (each layer compacted with ten tamps with a 40-lb spring tamper). Samples were tested after a 24-hour cure at 100 percent relative humidity and after a 24-hour cure at 100 percent relative humidity followed by a 24-hour water immersion. The strength of the untreated soils was 20 psi. Materials added to the soils were considered to have potential as stabilizers if they increased the strength from 20 to 100 psi or greater.

Each of the following additives were used:

(Continued on next page)

* Basic material 
2 percent phosphoric acid plus 0.5 percent sodium fluosilicate $\left(\mathrm{Na}_{2} \mathrm{SiF}_{6}\right) ; 0.5$ percent $\mathrm{Na}_{2} \mathrm{SiF}_{6}$ and 0.5 percent n-octylamine; and 0.5 percent $\mathrm{O}-\mathrm{P}_{2} \mathrm{O}_{5}$ and 0.5 percent $\mathrm{Na}_{2} \mathrm{SiF} 6$ and 0.5 percent n-octylamine.

3 percent phosphoric acid plus 0.5 percent sodium fluosilicate $\left(\mathrm{Na}_{2} \mathrm{SiF}_{6}\right) ; 1.0$ percent $\mathrm{Na}_{2} \mathrm{SiF}_{6}$ and 1 percent $\mathrm{n}$-octylamine; and 1 percent $\mathrm{Na}_{2} \mathrm{SiF}_{6}$ and 1.5 percent ferric chloride.

Effectivene 8 8: Lean clay - The 3 percent $\mathrm{H}_{3} \mathrm{PO}_{4}$ with 1 percent sodium fluosilicate and 1.5 percent ferric choride gave the best results ( $81 \mathrm{psi}$ dry cure and 72 psi after soak); however, the se values were below 100 psi.

Heavy clay - Same comments as for lean clay; however, strength values were 74 psi dry cure and 70 psi after soak. 


\section{Category*}

Acid

Basic Material

Rate of Material

Phosphoric acid $\left(\mathrm{H}_{3} \mathrm{PO}_{4}\right)$

2.0 and $3.0 \%$

Not given

Secondary Material

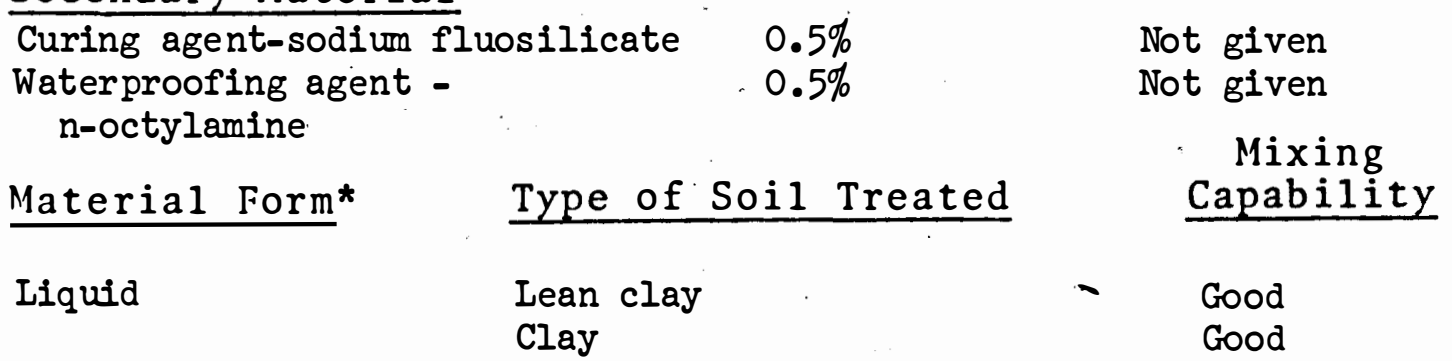

\begin{tabular}{|c|c|c|c|}
\hline Type of Test & $\begin{array}{l}\text { Purpose of } \\
\text { Material }\end{array}$ & $\begin{array}{l}\text { Effective } \\
\text { Strength } \\
\text { Increase }\end{array}$ & Effectiveness \\
\hline $\begin{array}{l}\text { Unconfined } \\
\text { compression }\end{array}$ & $\begin{array}{l}\text { Stabilizer } \\
\text { Waterproofer }\end{array}$ & See comments & See Comments \\
\hline
\end{tabular}

Total Material Cost

Per Cu Ft

of Treated Soil

Not given

Test Agency

WES
Test Report

Reference 25

\section{Comments:}

The untreated samples were not suitable for compression tests after 4 days cure followed by 4 days wetting by capillary action. The 3 percent phosphoric acid with the secondary materials was very effective as a stabilizer and waterproofing agent (300 psi unconfined. compression strength) on the lean clay soil. There was a big improvement with the clay soil; however, the materials were not effective as a stabilizer and waterproofer.

* Basic material 
Category*

Acid

Basic Material

Rate of Material

Cost

Phosphoric acid $\left(\mathrm{H}_{3} \mathrm{PO}_{4}\right)$

Varied ( 1 to $5 \%$ )

Not given

Secondary Material

Additives (see comments)

Material Form*

Type of Soil Treated

Mixing

Liquid

Clayey silt

Capability

Good

Effective

Purpose of Strength

Type of Test

Material

Increase Effectiveness

Unconfined

Stabilizer

See comments

Excellent

compression

Total Material Cost

Per Cu Ft

of Treated Soil

Not given

Test Agency

Test Report

MIT

Reference 38

Comments:

The basic material plus sodium fluosilicate was for stabilizing soil and octylamine and 2-ethyl hexylamine were added to check their waterproofing ability.

Additives

Sodium fluosilicate. 0.5 percent rate - the strength of soil treated only with this material is not effective.

When this material ( 0.5 percent) is used with 5 percent phosphoric acid, the strength of the 24-hour cure is approximately triple the strength where only $\mathrm{H}_{3} \mathrm{PO}_{4}$ is used. The strength after 24 hours and 24 hours water immersion closely parallels the 24-hour strength.

* Basic material 
Octylamine. (Rate varied from 0.05 to 2.0 percent). It was found that as little as 0.05 percent was adequate to waterproof the soil when used with 2 percent $\mathrm{H}_{3} \mathrm{PO}_{4}$ and sodium fluosilicate.

2-ethyl hexylamine. 0.2 percent was the most effective rate with 2 percent phosphoric acid and 0.5 percent sodium fluosilicate; 28 psi after 24 hours immersion, 198 psi after 24 hours humid cure, and 98 psi after 24 hours humid cure followed by 24 hours immersion and tests. However, this combination of materials was not as effective as that mentioned in Octylamine above. As the amount of the 2-ethyl hexylamine was increased, the strength decreased.

\section{Effectiveness:}

Sodium fluosilicate is very effective when used with phosphoric acid in increasing the strength of the treated samples.

Octylamine is more effective than 2-ethyl hexylamine in waterproofing soil treated with phosphoric acid and sodium fluosilicate. 
Category*

Acid

Basic Material

Phosphoric acid $\left(\mathrm{H}_{3} \mathrm{PO}_{4}\right)$
Rate of Material

$2 \%$

Octylamine

Ortho-rhombic phosphoric

Material Form*

Liquid
$0.5 \%$

$0.05 \%$

$\left(\mathrm{Na}_{2} \mathrm{SiF}_{6}\right)$

$$
0.05,0.10,0.25 \%
$$

Type of Soil Treated

Clayey silt
Cost

Not given
Not given

Not given

Not given

Capability

Good
Effective

Strength

Increase

See comments

Effectiveness

Type of Test

Unconfined compression
Purpose of

Stabilizer

Excellent

Total Material Cost

Per Cu Ft

of Treated Soil

Not given
Test Agency

MIT
Test Report

Reference 40

\section{Comments:}

The samples treated with the additives were compared to samples treated with only phosphoric acid. Tests were conducted after a 24 hour humid cure followed by an immersion in water for 24 hours. The combinations of additives which showed the most promise are given below.

(Continued on next page)

Basic material 


\begin{tabular}{|c|c|c|c|c|}
\hline $\begin{array}{r}\mathrm{Na}_{2} \mathrm{SiF}_{6} \\
(0.5 \%) \\
\end{array}$ & $\begin{array}{c}\text { Octylamine } \\
(0.05 \%) \\
\end{array}$ & $\begin{array}{l}\mathrm{O}-\mathrm{P}_{2} \mathrm{O}_{5} \\
(\%) \\
\end{array}$ & $\begin{array}{c}\text { Strength } \\
\text { psi } \\
\end{array}$ & $\begin{array}{l}\text { Strength Change Based on } \\
\text { Soil Treated with only } \\
\mathrm{H}_{3} \mathrm{PO}_{4}, \%\end{array}$ \\
\hline 0 & 0 & 0 & 200 & - \\
\hline Yes & 0 & 0 & 325 & +63 \\
\hline 0 & 0 & 0.05 & 340 & +70 \\
\hline Yes & Yes & 0.05 & 295 & +48 \\
\hline 0 & Yes & 0.05 & 425 & +113 \\
\hline Yes & 0 & 0.05 & 375 & +88 \\
\hline Yes & Yes & 0 & 350 & +75 \\
\hline
\end{tabular}

* The $\mathrm{Na}_{2} \mathrm{SiF}_{6}$ was mixed with the soil after the $0-\mathrm{P}_{2} \mathrm{O}_{5}$

Effectiveness: The most effective combination of additives was 0.05 percent octylamine plus 0.05 ortho-rhombic phosphoric anhydride (without sodium fluosilicate). 
Category* .

Acid

Basic Material

Phosphoric acid
Rate of Material

$5 \%$
Cost

Not given

Secondary Material

Chemical additives

Sodium fluosilicate

Rosinamine silicofluoride

Benzene phosphoric acid

Butyl acid phosphate

Phenyl acid phosphate

Isooctyl acid phosphate

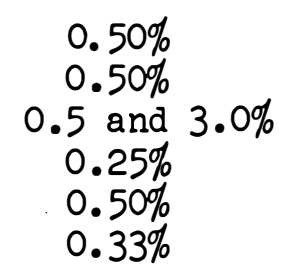

Type of Soil Treated

Material Form*

Liquid

Clayey silt

Effective

Purpose of

Type of Test

Unconfined

Strength

Increase

Stabilizer
Not given

Not given

Not given

Not given

Not given

Not given Mixing

Capability

Good

See comments . Excellent

Total Material Cost

Per Cu Ft

of Treated Soil

Not given

$\underline{\text { Test Agency }}$

Test Report

MIT

Reference 37

Comments:

See next page:

(Continued on next page)

* Basic material 
Compressive Strength After 24-hour Cure 100\% Relative Humidity and 24-hour Immersion,psi
Compressive

Percent Strength Increase after. Over Immediate Control Immersion,psi
Control (no additive)

Sodium fluosilicate

Rosinamine silicofluoride

Benzene phosphoric acid

(3 percent rate)

Butyl acid phosphate

Phenyl acid phosphate

Isooctyl acid phosphate
175

510

No test

250

210

135

185
$-$

191

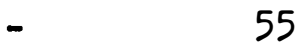

43

135

20

0

Negative $(-23) \quad 0$

6

0

Effectiveness: Sodium fluosilicate is an effective additive for improving phosphoric acid soil stabilization.

Benzene phosphoric acid when added to phosphoric acid was effective from the standpoint of strength and water resistance. 
Category*

Acid

Basic Material

Phosphoric acid

Secondary Material

Water

Material Form*

Liquid
Rate of Material

2, 5, and $10 \%$ on clayey silt Not given $2 \%$ on sandy clay

2 and $10 \%$ on clay
Cost

Mixing

Capability

Good

Good

Good
Clayey silt

Sandy clay

Clay

Effective

Strength

Type of Test

Unconfined

Compression
Purpose of Material

Stabilizer
Increase

See comments
Effectiveness

Excellent

Total Material Cost Per Cu Ft

of Treated Soil

Not given
Test Agency

MIT
Test Report

Reference 36

Comments:

Several methods or curing conditions were used; however, one week curing at room temperature and at 100 percent relative humidity followed by one week water immersion and then subjecting the samples to compressive tests was considered the most severe.

The treated samples were not compared to untreated samples.

The clayey silt treated samples at the 5 and 10 percent rate of phosphoric acid on dry soil and with a molding water content of 11 percent on dry soil were the only ones which showed promise as a stabilizer. After the curing conditions mentioned above, the 5 percent rate treated samples had a strength of $383 \mathrm{psi}$ and the 10 percent rate treated samples had a strength of 605 psi.

* Basic material 
Category*

Acid

Basic Material

Rate of Material

Cost

Phosphorus pentoxide

Not given

Secondary Material

\begin{tabular}{|c|c|c|c|}
\hline Material Form* & Type of So & il Treated & Capability \\
\hline Powder & $\begin{array}{l}\text { Sandy silt, } \\
\text { sandy clay, } \\
\text { clay }\end{array}$ & $\begin{array}{l}\text { clayey silt, } \\
\text { loess, and }\end{array}$ & Good \\
\hline Type of Test & $\begin{array}{c}\text { Purpose of } \\
\text { Material }\end{array}$ & $\begin{array}{l}\text { Effective } \\
\text { Strength } \\
\text { Increase }\end{array}$ & Effectiveness \\
\hline $\begin{array}{l}\text { Unconfined } \\
\text { compression }\end{array}$ & Stabilizer & See comments & $\begin{array}{c}\text { Excellent for } \\
\text { silt }\end{array}$ \\
\hline
\end{tabular}

Total Material Cost

Per Cu Ft

of Treated Soil

Not given

Test Agency

MIT
Test Report

Reference 36

\section{Comments:}

Tests were conducted on treated samples of 14 days cure and 7. days water immersion. Treated samples were not compared to untreated - samples.

Sandy silt and clayey silt soil samples treated with phosphorus pentoxide were the only samples which were considered to have retained any significant compressive strengths (282 and 153 psi, respectively) after tests.

* Basic material 
Category*

Acid

Basic Material

Rate of Material

Phosphorus pentoxide

3,5 , and $7 \%$

Cost

Not given

Secondary Material

Sodium fluosilicate

$0.5 \%$

Not given

Mixing

Material Form*

Type of Soil Treated

Lean clay

Capability

Powder

Good

\begin{tabular}{|c|c|c|c|}
\hline Type of Test & $\begin{array}{l}\text { Purpose of } \\
\text { Material } \\
\end{array}$ & $\begin{array}{l}\text { Effective } \\
\text { Strength } \\
\text { Increase } \\
\end{array}$ & Effectiveness \\
\hline $\begin{array}{l}\text { Unconfined } \\
\text { compression }\end{array}$ & Stabilizer & See comments & $\begin{array}{l}\text { Excellent (in } \\
\text { laboratory) } \\
\text { None (in field } \\
\text { tests) }\end{array}$ \\
\hline
\end{tabular}

Total Material Cost

Per Cu Ft

$\frac{\text { of Treated Soil }}{\text { Not given }} \frac{\text { Test Agency }}{\text { WES }} \frac{\text { Test Report }}{\text { Reference } 15}$

Comments:

Treated samples were compared to untreated samples (20 psi). Samples were prepared using the Harvard Miniature Compaction Apparatus in five layers (each layer was compacted with ten tamps of a 40-1b spring tamper). The samples were then cured for 24 hours under 100 percent relative humidity.

Each rate of basic material was used with the additive. The 5 percent rate gave the greatest (588 percent) strength increase and met the Category 2 requirements for stabilization.

Field traffic tests: A traffic test section (lean clay) was prepared and treated with 5 percent treatment of pentoxide and 0.5 percent sodium fluosilicate. However, the section failed before meeting stated requirements.

* Basic material 
Category: Asphalt 


\begin{tabular}{|c|c|c|c|}
\hline \multicolumn{4}{|l|}{ Category* } \\
\hline Basic Material & Rate & of Material & Cost \\
\hline \multicolumn{2}{|c|}{$\begin{array}{l}\text { Asphalt cutback (see } \\
\text { comments for various } \\
\text { ratios of asphalt to } \\
\text { solvent) } \\
\text { Secondary Material } \\
\end{array}$} & $5 \%$ & Not given \\
\hline \multicolumn{2}{|c|}{$\begin{array}{l}\text { Phosphorus pentoxide }\left(\mathrm{P}_{2} \mathrm{O}_{5}\right) \\
\text { (additive) }\end{array}$} & $3 \%$ & \multirow{2}{*}{$\begin{array}{l}\text { Not given } \\
\text { Mixing } \\
\text { Capability } \\
\end{array}$} \\
\hline Material Form* & Type of & Soil Treated & \\
\hline \multirow[t]{2}{*}{ Liquid } & \multicolumn{2}{|c|}{ Clayey silt } & Good \\
\hline & $\begin{array}{l}\text { Purpose of } \\
\text { Material }\end{array}$ & $\begin{array}{l}\text { Effective } \\
\text { Strength } \\
\text { Increase }\end{array}$ & Effectiveness \\
\hline $\begin{array}{l}\text { Unconfined } \\
\text { compression }\end{array}$ & Stabilizer & See comments & Excellent \\
\hline
\end{tabular}

Total Material Cost Per Cu Et of Treated Soil Test Agency Test Report

Not given MIT Reference 37

\section{Comments :}

Asphalt- 50-60 penetration was used at four degrees of cutback: $3: 1$, $2: 1,1: 1$, and $0.5: 1$ asphalt to gasoline. Cure conditions were 24 hours at 100 percent relative humidity and then samples were immersed in water for 24 hours. After immersion, the samples were subjected to compression tests.

Effectiveness: The samples without the additive did not have any significant strength. Asphalt cutback at the ratio of $3: 1$ (asphalt to gasoline) gave the best results with the additive, $\mathrm{P}_{2} \mathrm{O}_{5}$, when used to treat soil samples. As the amount of solvent increased, the strength values decreased. Also, the samples were harder to mix. The values for the cutback ratios $(3: 1,2: 1,1: 1$, and $0.5: 1)$ were $225,177,170$, and 143 psi, respectively.

* Basic material 
Category*

Asphalt

Basic Material

Asphalt cutback

(50-60 pen)
Rate of Material $5 \%$
Cost

Not given

Secondary Material

Solvents (see comments)

Phosphorus pentoxide $\left(\mathrm{P}_{2} \mathrm{O}_{5}\right)$ - additive Material Form*

Liquid
Type of Soil Treated

Clayey silt
Not given

Mixing

Capability

Good

\begin{tabular}{llll} 
Type of Test & $\begin{array}{l}\text { Purpose of } \\
\text { Material }\end{array}$ & $\begin{array}{l}\text { Effective } \\
\text { Strength } \\
\text { Increase }\end{array}$ & Effectiveness \\
\hline $\begin{array}{c}\text { Unconfined } \\
\text { Compression }\end{array}$ & Stabilizer & See coments & Excellent
\end{tabular}

Total Material Cost Per Cu Ft

$\frac{\text { of Treated Soil }}{\text { Not given }} \frac{\text { Test Agency }}{\text { MIT }} \frac{\text { Test Report }}{\text { Reference } 37}$

Comments:

Asphalt cutback composition $=1.43: 1$, asphalt to solvent (by volume).

Cure conditions - 24 hours cure at 100 percent relative humidity and then 24 hours immersion in water. Compressive tests then conducted.

Solvents used were: carbon disulfide, n-hexane, carbon tetrachloride, gasoline, and kerosene.

Effectiveness: The samples treated with asphalt and the various solvents without the additive had very little compressive strength. All samples treated with the various solvents plus the additive had good compressive strengths as follows:

(Continued on next page)

* Basic material 
n-hexine - 233 psi

Carbon disulfide - 194 psi

Gasoline - 177 psi

Carbon tetrachloride - 159 psi

Kerosene -76 psi 


\begin{tabular}{|c|c|c|}
\hline $\begin{array}{l}\text { Category* } \\
\text { Asphalt }\end{array}$ & & \\
\hline Basic Material & Rate of Material & Cost \\
\hline $\begin{array}{l}\text { Asphalt cutback (see } \\
\text { comments for various } \\
\text { penetration numbers) }\end{array}$ & $5 \%$ & Not given \\
\hline Secondary Material & & \\
\hline $\begin{array}{l}\text { Phosphorus pentoxide } \\
\left(\mathrm{P}_{2} \mathrm{O}_{5}\right) \text { (additive) }\end{array}$ & $3 \%$ & Not given \\
\hline Material Form* & Type of Soil Treated & $\begin{array}{c}\text { Mixing } \\
\text { Capability } \\
\end{array}$ \\
\hline Liquid & Clayey silt & Good \\
\hline
\end{tabular}

$\begin{array}{cccc}\text { Type of Test } & \begin{array}{c}\text { Purpose of } \\ \text { Material }\end{array} & \begin{array}{l}\text { Effective } \\ \text { Strength } \\ \text { Increase }\end{array} & \text { Effectiveness } \\ \begin{array}{c}\text { Unconfined } \\ \text { compressive }\end{array} & \text { Stabilizer } & \text { See comments } & \text { Excellent }\end{array}$

Total Material Cost Per $\mathrm{Cu} \mathrm{Ft}$ of Treated Soil

Not given

Test Agency

MIT
Test Report

Reference 37

\section{Comments :}

Cure conditions - 24 hours at 100 percent relative humidity followed by 24 hours immersion.

Asphalt cutback composition $=2: 1$ asphalt to gasoline cutback asphalt with various penetration numbers: 100-120, 85-100, 65-70, and 50-60 were tested with samples without additives and with additives $\left(\mathrm{P}_{2} \mathrm{O}_{5}\right)$.

Effectiveness: The samples without additive when subject to the compressive tests had no significatn compressive strength, whereas the strength of all treated samples with the additive, $\mathrm{P}_{2} \mathrm{O}_{5}$, was 124 to 177 psi. The lower the penetration number, the higher the strength was for these samples. The samples tested with 100-120 pen asphalt had asphalt strength of 124 psi, and those treated with 50-60 pen asphalt had strength of 177 psi.

* Basic material 
Category*

Asphalt

Basic Material

Rate of Material

Cost

Cutback asphalt

$5 \%$

Not given

Straight run, cracked,

and blown

Secondary Material

Additives (see below)

Material Form*

Type of Soil Treated

Mixing

Capability

Iiquid

Clayey silt

Good

Effective

Purpose of Strength

$\frac{\text { Type of Test }}{\text { Unconfined }} \frac{\text { Material }}{\text { Stabilizer }} \quad \frac{\text { Increase }}{\text { See comments }} \quad \frac{\text { Effectiveness }}{\text { Excellent }}$

compressive

Total Material Cost

Per Cu Ft

of Treated Soil

Not given

Test Agency

MIT
Test Report.

Reference 35

Comments:

The following additives were tested with cutback asphalts. Cure time

was 14 days and rewet strength was checked after 7 days water immersion.

(Continued on next page)

* Basic material 
Straight Run

Epon 828 (10 percent)

plus diethylene

triamine (2 percent)

Toluene diisocyanate

( 5 percent) plus

ethylene glycol

(5 percent)

Toluene diisoycanate

( 5 percent) plus

diethylene triamine

( 5 percent)

Epon 828 (10 percent)

plus $\mathrm{BF}_{3}$ (2 percent

plus diethylene triamine

(2 percento

Toluene diisocyanate

(5 percent) plus

ethylene glycol

(5 percent) plus $\mathrm{BF}_{3}$

(2 percent)

Styrene (20 percent)

plus $\mathrm{BF}_{3}$ (10 percent)

Styrene (20 percent

plus $\mathrm{BF}_{3}$ (10 percent)

plus Benzoyl peroxide

plus dimethylaniline

(2 percent)

$\mathrm{BF}_{3}(5,10$, and 20

percent-)

$\mathrm{BF}_{3}$ (10 percent) plus

acrylonitrile (10

percent)
Cracked

Toluene diisocyanate

(10 percent)

$\mathrm{BF}_{3}$ (2 and 5 percent)

$\mathrm{H}_{2} \mathrm{SO}_{4}$ (Conc)

( 5 percent)

Styrene (10 percent)

plus $\mathrm{BF}_{3}$ (5 percent)

Acrylonitrile (10

percent) plus $\mathrm{BF}_{3}$

(5 percent)

Acrylonitrile

(10 percent) plus

$\mathrm{H}_{2} \mathrm{SO}_{4}$ (Conc)

( 5 percent)

Triphenyl methane

triisocyanate

(2 percent)

Toluene

diisocyanate (10

percent)

Diphenyl methane

diisocyanate (10

percent)

Diethylene

triamine (10

percent)

Epon 828 (10 percent)

plus diethylene

triamine (2 percent)

Methyle sulfate (10

percent)
Blown

Toluene diisocyanate

(10 percent)

$\mathrm{BF}_{3}$ (2 and 5 percent)

$\mathrm{H}_{2} \mathrm{SO}_{4}$ (Conc)

(5 percent)

Styrene (10 percent)

plus $\mathrm{BF}_{3}$ ( 5 percent)

Acrylonitrile

(10 percent)

Triphenyl methane triisocyanate (2 percent)

Toluene

diisocyanate ( 10

percent)

Diphenyl methane diisocyanate ( 10 percent)

Epon 828 (10

percent) plus

diethylene triamine

(2 percent)

Methyl sulfate (10 percent) 
It was concluded in the report that any additive capable of increasing the rewet compressive strength to a value of $150 \mathrm{psi}$ or greater would merit further study.

Several of the additives fall into this category. Given below are the additives which appeared beneficial to asphalt cutback stabiliation (and in order of effectiveness).

Straight Run

Toluene diisocyanate

(10 percent)

$\mathrm{P}_{2} \mathrm{O}_{5}(20$ percent $)$

Diphenyl methand

Diisocyanate

(10 percent)

Epon 828 (10

percent plus

diethylene triamine

(2 percent)

Methyl sulfate

(10 percent)

Triphenyl methane

Triisocyanate

(2 percent)

\begin{tabular}{ll}
\multicolumn{1}{c}{ Cracked } & \\
\cline { 2 - 2 } $\begin{array}{l}\text { Diphenyl methane } \\
\text { diisocyanate (10 }\end{array}$ & $\begin{array}{l}\text { Diphenyl methane } \\
\text { dilsocyanate (10 } \\
\text { percent) }\end{array}$ \\
$\begin{array}{ll}\text { Toluene diisocyanate } \\
\text { (10 percent) }\end{array}$ & $\begin{array}{l}\text { Epon } 828 \text { (10 } \\
\text { percent plus } \\
\text { diethylent triamine } \\
\text { (2 percent) }\end{array}$ \\
$\begin{array}{l}\text { Triphenyl methane } \\
\text { (2 percent) }\end{array}$ & $\begin{array}{l}\text { Toluene diisocyanate } \\
\text { (10 percent) }\end{array}$ \\
$\begin{array}{l}\text { Epon } 828 \text { (10 percent) } \\
\text { plus diethylene }\end{array}$ & \\
triamine (2 percent) &
\end{tabular}

Further work was conducted with the asphalts and various additives as mentioned above. The results of the work led to the following conclusions :

a. Modification of asphalt cutbacks with reactive chemical compounds such as $\mathrm{P}_{2} \mathrm{O}_{5}$ or toluene or diphenyl methane diisocyanate (at concentrations of 10 percent on the asphalt or below). significantly improves cutback stabilization of fine-graned soils, as measured by evelation of compressive strength after seven days water immersion. $\mathrm{P}_{2} \mathrm{O}_{5}$ also markedly accelerates the development of water resistance of stabilized soil during drying and/or curing.

b. There is a general correlation between rewet strength and volatiles content of the specimen at the time of test. From this correlation, it has been deduced that asphalt, irrespective of its method of incorporation with soil or its chemical alteration, functions primarily as a waterproofing agent for soil, the various additives and improved methods if incorporation merely enhancing its characteristic waterproofing ability. 


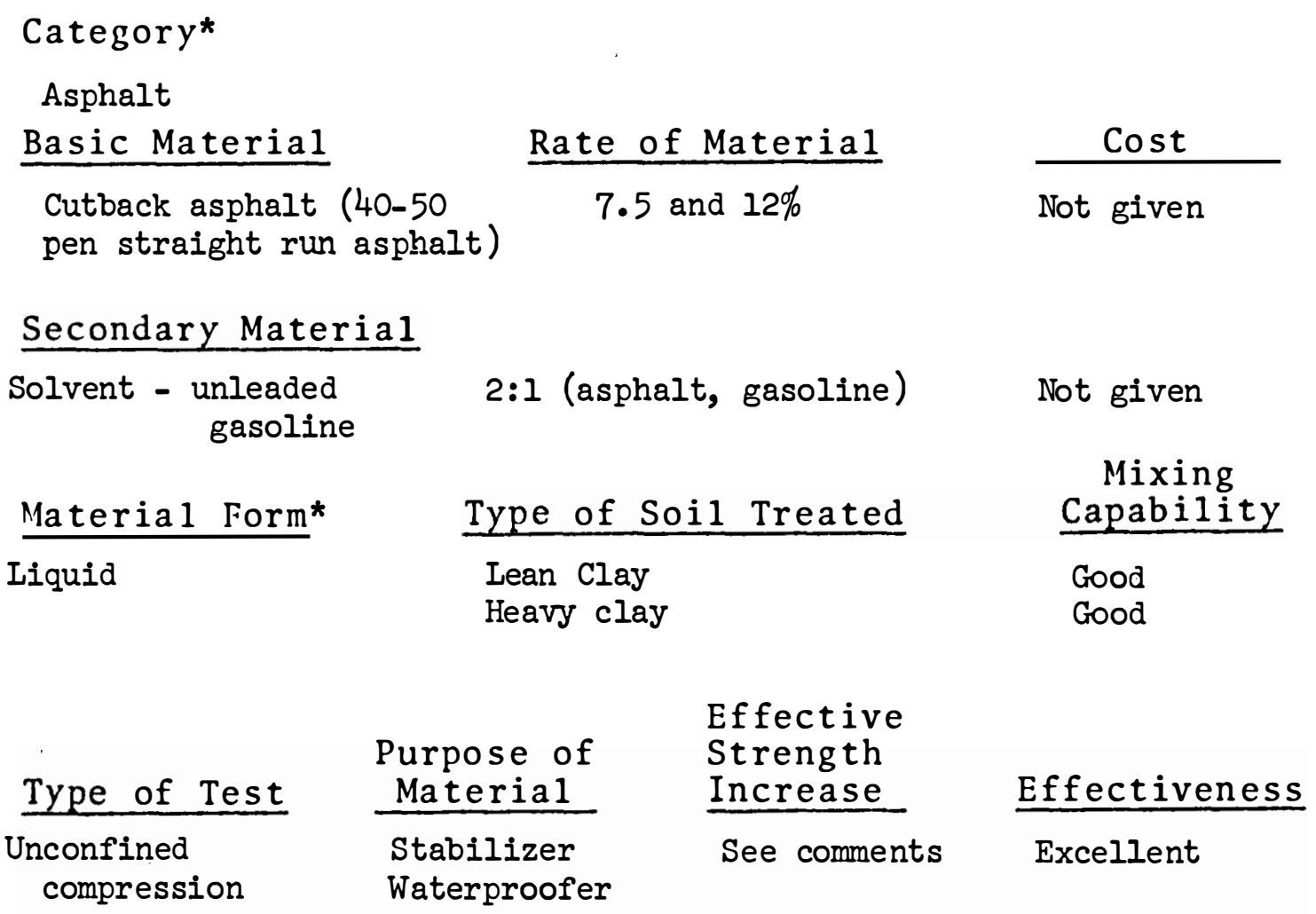

Total Material Cost Per $\mathrm{Cu} \mathrm{Ft}$

$\frac{\text { of Treated Soil }}{\text { Not given }} \quad \frac{\text { Test Agency }}{\text { WES }} \frac{\text { Test Report }}{\text { Reference 25 }}$

Comments:

Untreated samples were not suitable for compression tests after 4 days dry cure followed by 4 days wetting by capillary action.

Effectiveness: Lean clay - Both rates of asphalt were effective in waterproofing and stabilizing the samples with no significant benefits with the higher rate of asphalt.

Heavy clay - Same as for lean clay.

* Basic material 


\section{Category*}

Asphalt

Basic Material

Cutback asphalt (40-50

pen straight run asphalt)

Secondary Material

Solvent - unleaded

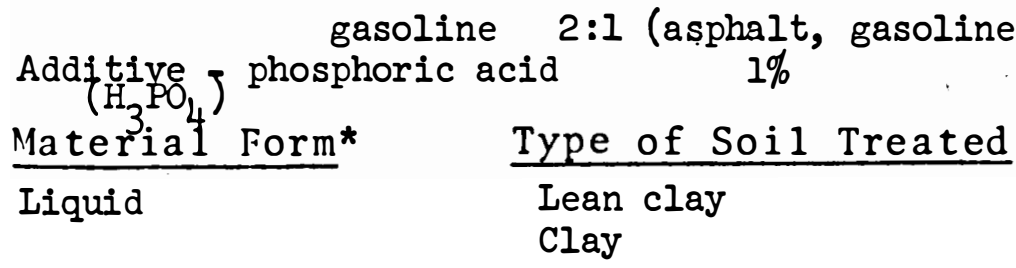

Effective

Purpose of

Type of Test

Unconfined

compression
Rate of Material

$$
7.5 \%
$$

Cost

Not given
Not given

Not given Mixing

Capability

Good

Good

Total Material Cost

Per Cu Ft

of Treated Soil

Not given

\section{Test Agency}

WES
Test Report

Reference 25

\section{Comments:}

Untreated samples were not suitable for compression tests after 4 days dry cure followed by 4 days wetting by capillary action.

Effectiveness: Lean clay - The combination of materials was effective as a stabilizer and waterproofer; however, the combination was not as effective as asphalt only treatment.

Clay - The combination of materials was only slightly effective; however, the strength of asphalt only treated samples was twice that of the samples treated with the combination of materials.

* Basic material 
Ca tegory*

Asphalt

Basic Material

Cutback asphalt (40-

50 pen straight run

asphalt)
Rate of Material

$1.5 \%$

Cost

Not given

Secondary Material

Solvent - unleaded gasoline

Additives - phosphoric acid

plus alky dimethy benzyl

ammonium chloride

Material Form*

Liquid

Type of Soil Treated

Lean clay

Clay

2:1 (asphalt, gasoline) $1.0 \%$

Not given

Not given

Not given

Mixing

Capability

Good

Good

\begin{tabular}{|c|c|c|c|}
\hline Type of Test & $\begin{array}{l}\text { Purpose of } \\
\text { Material }\end{array}$ & $\begin{array}{l}\text { Effective } \\
\text { Strength } \\
\text { Increase }\end{array}$ & Effectiveness \\
\hline $\begin{array}{l}\text { Unconfined } \\
\text { compression }\end{array}$ & $\begin{array}{l}\text { Stabilizer } \\
\text { Waterproofer }\end{array}$ & None & None \\
\hline
\end{tabular}

Total Material Cost

Per Cu Ft

$\frac{\text { of Treated Soil }}{\text { Not given }} \frac{\text { Test Agency }}{\text { WES }} \frac{\text { Test Report }}{\text { Reference 25 }}$

Comments:

The samples treated with asphalt only gave much better results than those treated with the combination of materials.

* Basic material 


\begin{tabular}{|c|c|c|c|}
\hline \multicolumn{4}{|l|}{$\begin{array}{l}\text { Ca tegory* } \\
\text { Asphalt }\end{array}$} \\
\hline Basic Material & \multicolumn{2}{|c|}{ Rate of Material } & Cost \\
\hline $\begin{array}{l}\text { Cutback asphalt ( } 4 \\
\text { pen straight mun a }\end{array}$ & \multicolumn{2}{|c|}{$7.5 \%$} & Not given \\
\hline \multicolumn{2}{|c|}{$\begin{array}{l}\text { Solvent - unleaded gasoline } \\
\text { Additives }- \text { phosphoric } \\
\text { acid }\left(\mathrm{H}_{3} \mathrm{PO}_{4}\right) \\
\text { plus laury } \mathrm{I} \text { amine }\end{array}$} & $\begin{array}{l}\text { asphalt, gasoline) } \\
0 \% \text { p } \\
10 \%\end{array}$ & \multirow{2}{*}{$\begin{array}{l}\text { Not given } \\
\text { Not given } \\
\text { " Mixing } \\
\text { Capability }\end{array}$} \\
\hline Material Form* & Type of & il Treated & \\
\hline Liquid & \multicolumn{2}{|c|}{$\begin{array}{l}\text { Lean clay } \\
\text { Clay }\end{array}$} & $\begin{array}{l}\text { Good } \\
\text { Good }\end{array}$ \\
\hline Type of Test & $\begin{array}{l}\text { Purpose of } \\
\text { Material }\end{array}$ & $\begin{array}{l}\text { Effective } \\
\text { Strength } \\
\text { Increase }\end{array}$ & Effectiveness \\
\hline $\begin{array}{l}\text { Unconfined } \\
\text { compression }\end{array}$ & $\begin{array}{l}\text { Stabilizer } \\
\text { Waterproofer }\end{array}$ & None & None \\
\hline
\end{tabular}

Total Material Cost

Per Cu Ft

of Treated Soil

Not given

Test Agency

WES
Test Report

Reference 25

\section{Comments:}

Untreated samples were not suitable for compression tests after 4 days dry cure followed by 4 days wetting by capillary action. Samples treated with only asphalt gave much better results.-

* Basic material 


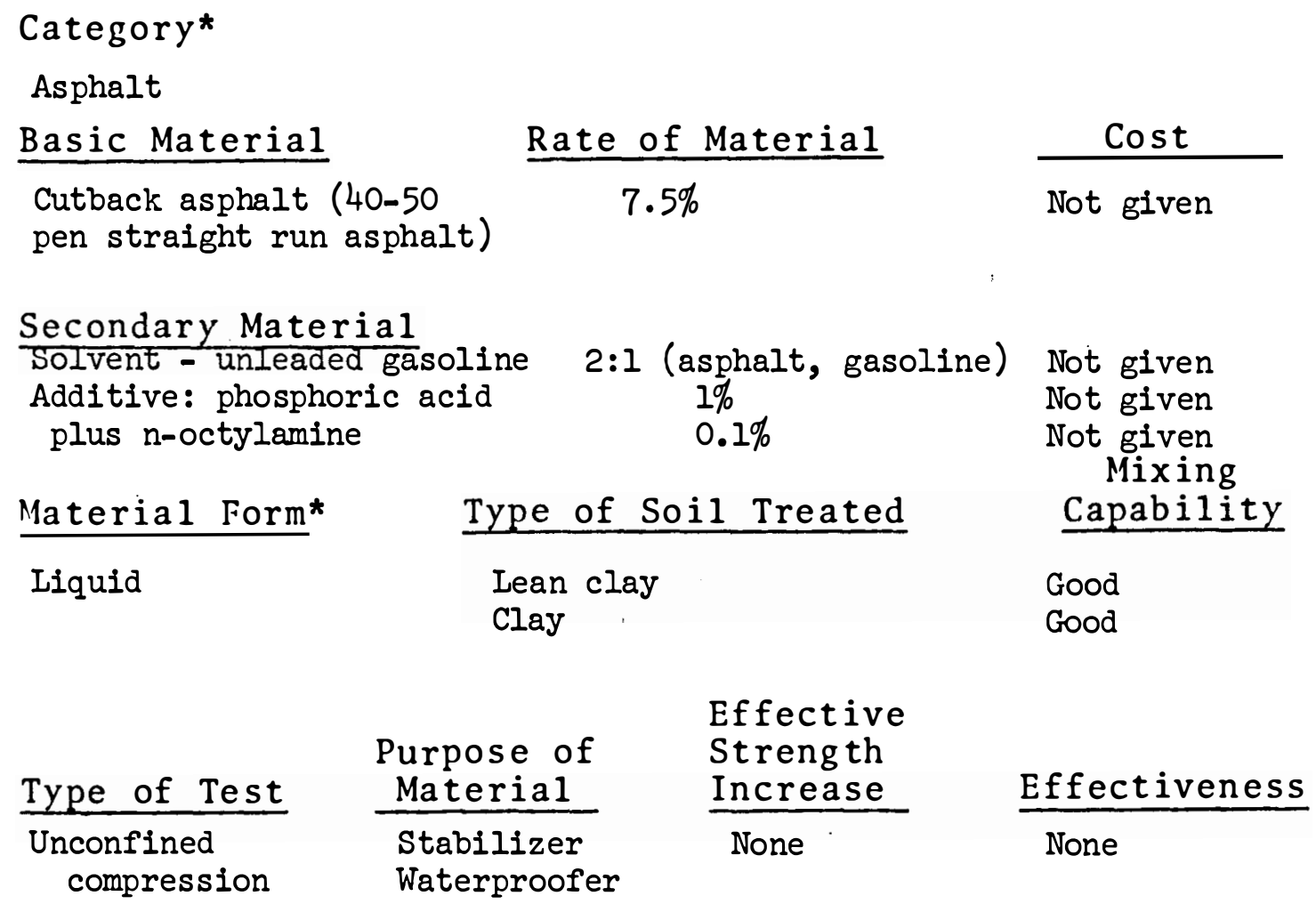

Total Material Cost Per $\mathrm{Cu} \mathrm{Ft}$

$\frac{\text { of Treated Soil }}{\text { Not given }} \quad \frac{\text { Test Agency }}{\text { WES }} \frac{\text { Test Report }}{\text { Reference 25 }}$

\section{Comments:}

The asphalt only treated samples gave much better results than the combination of materials.

* Basic material 
Category*

Asphalt

Basic Material

Cutback asphalt (40-50

pen straight run asphalt)

Secondary Material

\section{Solvent - unleaded gasoline Additives -}

Phosphoric acid $\left(\mathrm{H}_{3} \mathrm{PO}_{4}\right)$

plus octadecyl amine acetate

Material Form*

Liquid

Type of Soil Treated

Lean clay

Clay

2:1 (asphalt, gasoline) Not given

\author{
$1.0 \%$
}

$0.10 \%$

Not given

Mixing

Capability

Good

Good

\begin{tabular}{|c|c|c|c|}
\hline Type of Test & $\begin{array}{c}\text { Purpose of } \\
\text { Materia } 1 \\
\end{array}$ & $\begin{array}{l}\text { Effective } \\
\text { Strength } \\
\text { Increase } \\
\end{array}$ & Effectiveness \\
\hline $\begin{array}{l}\text { Unconfined } \\
\text { compression }\end{array}$ & $\begin{array}{l}\text { Stabilizer } \\
\text { Waterproofer }\end{array}$ & None & None \\
\hline
\end{tabular}

Total Material Cost

Per Cu Ft

of Treated Soil

Test Agency

Test Report

Not given

WES

Reference 25

Comments:

Asphalt only treated samples were much more effective than the combination of materials.

* Basic material 


\section{Category*}

Asphalt

Basic Material

Cutback asphalt (40-50

pen straight run asphalt)
Rate of Material

7.5 and $12 \%$
Cost

Not given

Secondary Material

Solvent - unleaded gasoline 2:I (asphalt, gasoline) Not given

Additive: phosphorus $3 \%$ pentoxide

Material Form*

Type of Soil Treated

Liquid

Lean Clay

Clay

Not given

Mixing

Capability

Good

Good

Effective

Purpose of Strength

$\frac{\text { Type of Test }}{\text { Unconfined }}$

compression

Material

Increase

See comments

$\frac{\text { Effectiveness }}{\text { See comments }}$

Total Material Cost

Per Cu Ft

of Treated Soil

Not given

Test Agency

Test Report

WES

Reference 25

Comments:

Untreated samples were not suitable for compression tests after 4 days dry cure followed by 4 days wetting by capillary action.

Effectiveness: Lean clay - Samples treated with both rates of asphalt with additive were effective in waterproofing and stabilizing the samples. However, the 7.5 percent asphalt gave the best results of the two asphalt rates and this strength was significantly better than asphalt only treated samples.

Clay - Both rates of asphalt with additive were effective; however, greater strength values were obtained with only the basic material.

* Basic material 
Category*

Asphalt

Basic Material

Rate of Material

7.5 and $12 \%$
Cost

Not given

Cutback asphalt (40-50

straight run asphalt)

Secondary Material Solvent - unleaded gasoline 2:1 (asphalt, gasoline) Not given
Additive:

Thosphorus pentoxide

$\left(\mathrm{P}_{2} \mathrm{O}_{5}\right)$

$0.25 \%$ with $7.5 \%$ asphalt Not given

$0.40 \%$ with $12 \%$ asphalt

Material Form*

Iiquid

Type of Soil Treated

Mixing

Capability

Lean clay

Good

Clay

Good

\begin{tabular}{|c|c|c|c|}
\hline Type of Test & $\begin{array}{c}\text { Purpose of } \\
\text { Material }\end{array}$ & $\begin{array}{l}\text { Effective } \\
\text { Strength } \\
\text { Increase }\end{array}$ & Effectiveness \\
\hline $\begin{array}{l}\text { Unconfined } \\
\text { compression }\end{array}$ & $\begin{array}{l}\text { Stabilizer } \\
\text { Waterproofer }\end{array}$ & See comments & See comments \\
\hline
\end{tabular}

Total Material Cost

Per Cu Ft

of Treated Soil

Test Agency

Test Report

Not given

WES

Reference 25

Comments:

Untreated samples were not suitable for compression tests after 4 days dry cure followed by 4 days wetting by capillary action.

Effectiveness: Lean clay - Both rates of asphalt with the additive were effective in waterproofing and stabilizing the samples. 'However, the strengths of the samples with the 0.25 percent $\mathrm{P}_{2} \mathrm{O}_{5}$ were less than those with 7.5 percent asphalt only. The samples with 12 percent asphalt and 0.4 percent $\mathrm{P}_{2} \mathrm{O}_{5}$ had strength somewhat higher than the asphalt only treated sampless?

Clay - Samples treated with both rates of asphalt with additive were effective in waterproofing and stabilizing; however, the strength values were less than those for 7.5 and 12 percent asphalt only.

* Basic material 


\section{Category*}

Asphalt

Basic Material

Cutback asphalt (40-50

Rate of Material

7.5 and $12 \%$

Cost

pen straight run asphalt)

Secondary Material

Solvent - unleaded gasoline 2:1 (asphalt, gasoline) Not given Additives (see comments)

$\frac{\text { Material Form }}{\text { Liquid }} \quad \frac{\text { Type of Soil Treated }}{\begin{array}{l}\text { Lean clay } \\ \text { Clay }\end{array}} \quad \begin{gathered}\begin{array}{c}\text { Mixing } \\ \text { Capability }\end{array} \\ \begin{array}{c}\text { Good } \\ \text { Good }\end{array}\end{gathered}$

$\begin{array}{llll}\text { Type of Test } & \begin{array}{l}\text { Purpose of } \\ \text { Material }\end{array} & \begin{array}{l}\text { Effective } \\ \text { Strength } \\ \text { Increase }\end{array} & \text { Enconfined } \\ \text { compression } & \begin{array}{l}\text { Stabilizer } \\ \text { Waterproofer }\end{array} & \text { See commets }\end{array} \quad$ See comments

Total Material Cost

Per $\mathrm{Cu} \mathrm{Ft}$

of Treated Soil

Test Agency

Test Report

Not given

WES

Reference 25

Comments:

Untreated samples were not suitable for compression tests after 4 days dry cure followed by 4 days wetting by capillary action.

Additives:

$7.5 \%$ asphalt and $0.25 \%$ phosphorus pentoxide $\left(\mathrm{P}_{2} \mathrm{O}_{5}\right)$ plus $0.10 \%$ alkyl dimethyl benzyl ammonium chloride (ADBAC)

$7.5 \%$ asphalt and $3.0 \% \mathrm{P}_{2} \mathrm{O}_{5}$ plus $0.2 \%$ ADBAC

(Continued on next page)

Basic material 
$12 \%$ asphalt and $0.40 \% \mathrm{P}_{2} \mathrm{O}_{5}$ plus $0.10 \% \mathrm{ADBAC}$

$12 \%$ asphalt and $3.0 \% \mathrm{P}_{2} \mathrm{O}_{5}$ plus $0.2 \%$ ADBAC

Effectiveness: Lean clay - Both rates of asphalt with additives (all rates) were effective in waterproofing and stabilizing samples. However, 7.5 percent asphalt with 3.0 percent $\mathrm{P}_{2} \mathrm{O}_{5}$ and 0.10 percent ADBAC was more effective than asphalt alone.. The other combinations of materials were not as effective as asphalt only.

Clay - 7.5 percent asphalt with 3.0 percent $\mathrm{P}_{2} \mathrm{O}_{5}$ plus 0.10 percent ADBAC was the most effective combination as was slighly more effective than only 7.5 percent asphalt. The other combinations of materials were not as effective as asphalt only at the two different rates. 
Category*

Asphalt

Basic Material Rate of Material

Cost

Cutback asphalt (40-

50 pen straight run asphalt)

7.5 and $12 \%$

Not given

Secondary Material

Solvent - unleaded 2:1 (asphalt, gasoline) Not given gasoline

Additives (see comments)

Material Form*

Liquid

$\frac{\text { Type of Soil Treated }}{\text { Lean clay }}$

Clay
Mixing

Capability

Good

Good

$\begin{array}{llll}\text { Type of Test } & \begin{array}{l}\text { Purpose of } \\ \text { Material }\end{array} & \begin{array}{l}\text { Effective } \\ \text { Strength } \\ \text { Increase }\end{array} & \text { Sefectiveness } \\ \begin{array}{c}\text { Unconfined } \\ \text { compression }\end{array} & \begin{array}{l}\text { Stabilizer } \\ \text { Waterproofer }\end{array} & \text { See comments } & \text { See comments }\end{array}$

Total Material Cost

Per Cu Ft

of Treated Soil

Not given

Test Agency

WES
Test Report

Reference 25

Comments:

Untreated samples were not suitable for compression tests after 4 days dry cure followed by 4 days wetting by capillary action.

Additives:

7.5 percent asphalt and 0.25 percent phosphorus pentoxide plus

0.10 percent lauryl amine.

3.5 percent asphalt and 3.0 percent phosphorus pentoxide $\left(\mathrm{p}_{2} \mathrm{O}_{5}\right)$ plus 0.2 percent laurly amine.

12 percent asphalt and 0.4 percent phosphorus pentoxide plus

0.1 percent lauryl amine.

(Continued on next page)

Basic material 
12 percent asphalt and 3.0 percent phosphorus pentoxide plus 0.2 percent lauryl amine.

Effectiveness: Lean clay - The asphalt (at both rates) with the additives (all rates) were effective in waterproofing and stabilizing the samples. The 7.5 percent asphalt with 3.0 percent $\mathrm{P}_{2} \mathrm{O}_{5}$ and 0.2 percent lauryl amine was the most effective combination of materials. This combination was also more effective that either rate of asphalt alone.

Clay - Treatment with only asphalt (both rates) was more effective than treatment with asphalt plus additives. 
Category*

Asphalt

Basic Material

Rate of Material

Cost

Cutback asphalt (40-50

7.5 and $12 \%$

Not given

pen straight run asphalt)

Secondary Material

Solvent - unleaded gasoline 2:1 (asphalt, gasoline) Not given

Material Form*

Type of Soil Treated

Mixing

Liquid

Lean clay

Capability

Clay

Good

Good

\begin{tabular}{|c|c|c|c|}
\hline Type of Test & $\begin{array}{l}\text { Purpose of } \\
\text { Material } \\
\end{array}$ & $\begin{array}{l}\text { Effective } \\
\text { Strength } \\
\text { Increase } \\
\end{array}$ & Effectiveness \\
\hline $\begin{array}{l}\text { Unconfined } \\
\text { compression }\end{array}$ & $\begin{array}{l}\text { Stabilizer } \\
\text { Waterproofer }\end{array}$ & See comments & See comments \\
\hline
\end{tabular}

Total Material Cost

Per Cu Ft

$\frac{\text { of Treated Soil }}{\text { Not given }} \frac{\text { Test Agency }}{\text { WES }} \frac{\text { Test Report }}{\text { Reference 25 }}$

\section{Comments:}

Untreated samples were not suitable for compression tests after 4 days dry cure followed by 4 days wetting by capillary action.

\section{Additive 8}

7.5\% asphalt and $0.25 \%$ phosphorus pentoxide $\left(\mathrm{P}_{2} \mathrm{O}_{5}\right)$ plus $0.1 \%$ n-octylamine

7.5\% asphalt and $3.0 \% \mathrm{P}_{2} \mathrm{O}_{5}$ plus $0.20 \%$ n-octylamine

(Continued on next page)

* Basic material 
$12 \%$ a sphalt and $0.4 \% \mathrm{P}_{2} \mathrm{O}_{5}$ plus $0.1 \%$ n-octylamine

$12 \%$ a sphalt and $3.0 \% \mathrm{P}_{2} \mathrm{O}_{5}$ plus $0.2 \%$ n-octylamine

Effectiveness: Lean clay - Both rates of a sphalt with additives (all rates) we re effective in waterproofing and stabilizing the samples; however, the only combination that gave any great increase over asphalt only was the following:

7.5 percent a sphalt plus 3.0 percent $\mathrm{P}_{2} \mathrm{O}_{5}$ and 0.20 percent n-octyla mine.

Clay - Both rates of asphalt with additives (all rates) were effective in waterproofing and stabilizing the samples; however, the only combination that gave any increase over asphalt only was the following: 7.5 percent asphalt plus 3 percent $\mathrm{P}_{2} \mathrm{O}_{5}$ and 0.2 percent n-octylamine. 
Category*

Asphalt

Basic Material

Rate of Material

Cost

Cutback asphalt

(40-50 pen straight run

7.5 and $12 \%$

Not given asphalt)

Secondary Material

Solvent - unleaded gasoline

2:1 (asphalt, gasoline) Not given

Additives (see comments)

Material Form*

Iiquid

$\frac{\text { Type of Soil Treated }}{\text { Lean Clay }}$

Mixing

Capability

Good

Clay

Good

\begin{tabular}{|c|c|c|c|}
\hline Type & $\begin{array}{l}\text { Purpose of } \\
\text { Material }\end{array}$ & $\begin{array}{l}\text { Effective } \\
\text { Strength } \\
\text { Increase }\end{array}$ & Effectiveness \\
\hline $\begin{array}{l}\text { Unconfined } \\
\text { compression }\end{array}$ & $\begin{array}{l}\text { Stabilizer } \\
\text { Waterproofer }\end{array}$ & See comments & See Comments \\
\hline
\end{tabular}

Total Material Cost

Per $\mathrm{Cu} \mathrm{Ft}$

$\frac{\text { of Treated Soil }}{\text { Not given }}$

$\frac{\text { Test Agency }}{\text { WES }}$

$\frac{\text { Test Report }}{\text { Reference } 25}$

Comments:

Untreated samples were not suitable for compression tests after 4 days dry cure followed by 4 days wetting by capillary action.

Additives:

$7.5 \%$ asphalt and $0.25 \%$ phosphorus pentoxide $\left(\mathrm{F}_{2} \mathrm{O}_{5}\right)$ plus $0.1 \%$ octadecyl amine acetate

$7.5 \%$ asphalt and $3.0 \% \mathrm{P}_{2} \mathrm{O}_{5}$ plus $0.2 \%$ ocadecyl amine acetate

(Continued on next page)

* Basic material 
$12 \%$ asphalt and $0.4 \% \mathrm{P}_{2} \mathrm{O}_{5}$ plus $0.1 \%$ octadecyl amine acetate $12 \%$ asphalt and $3.0 \% \mathrm{P}_{2} \mathrm{O}_{5}$ plus $0.2 \%$ octadecyl amine acetate Effectiveness: Lean clay - Asphalt at both percentages with the additives (all rates) were effective in waterproofing and stabilizing the samples. The 7.5 percent asphalt with 3 percent $\mathrm{P}_{2} \mathrm{O}_{5}$ and 0.2 percent octadecyl amine acetate was the most effective combination. This combination was more effective than iither rate of asphalt alone.

Clay - The 7.5 percent rate of asphalt with 3.0 percent $\mathrm{P}_{2} \mathrm{O}_{5}$ plus 0.2 percent octadecyl amine acetate was very effective in stabilizing and waterproofing the samples. Treatment with only 12 percent asphalt was more effective that treatment with 12 percent asphalt plus additives. 
Category*

Asphalt

Basic Material

Rate of Material

Cost

Straight run

See comments

Not given

asphalt

Secondary Material

Chemical additives (see

comments )

Material Form*

Type of Soil Treated

Mixing

Iiquid

Clayey silt

Capability

Not given

\begin{tabular}{|c|c|c|c|}
\hline Type of Test & $\begin{array}{c}\text { Purpose of } \\
\text { Material } \\
\end{array}$ & $\begin{array}{l}\text { Effective } \\
\text { Strength } \\
\text { Increase } \\
\end{array}$ & Effectiveness \\
\hline $\begin{array}{l}\text { Unconfined } \\
\text { compression }\end{array}$ & Stabilizer & See comments & Excellent \\
\hline
\end{tabular}

Total Material Cost

Per Cu Ft

of Treated Soil

Not given

Test Agency

MIT
Test Report

Reference 36

Comments:

The following chemical additives were each used with a 5 percent asphalt cutback (composition 2:1 asphalt to gasoline) with a mixing water content of 11 percent on dry soil.

Benzene phosphoric acid

(10 percent)

$\mathrm{PCl}_{3}$ (10 percent)

$\mathrm{POCl}_{3}$ (10 percent)

(Continued on next page)

* Basic material
85 percent $\mathrm{H}_{3} \mathrm{PO}_{4}$ (10 percent)

$\mathrm{PCl}_{5}$ (10 percent)

Yellow P (10 percent) + Armeen 18 DAc (2 percent) $+\mathrm{CS}_{2}$ (25 percent) 


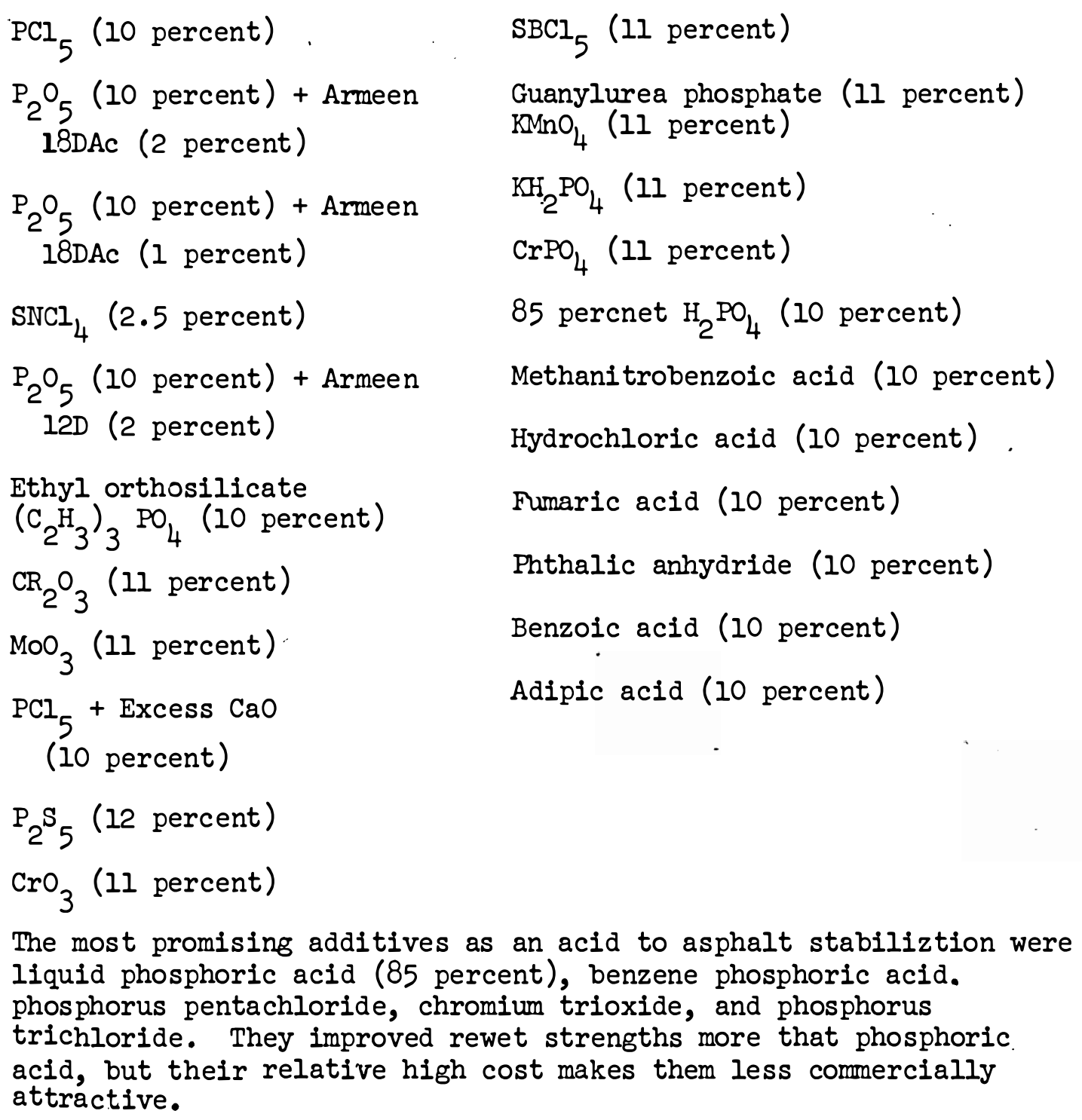

The most promising additives as an acid to asphalt stabiliztion were liquid phosphoric acid ( 85 percent), benzene phosphoric acid. phosphorus pentachloride, chromium trioxide, and phosphorus trichloride. They improved rewet strengths more that phosphoric. acid, but their relative high cost makes them less commercially attractive. 
Category*

Asphalt

Basic Material

Straight run asphalt

(40-50 pen)

Secondary Material

\section{Emuls ifying agents:}

Duomeen $T$

Hyd rochloric acid

Solvent - ga soline

Additive

Phosphoric acid

Material Form*

Liquid

$\frac{\text { Type of Test }}{\begin{array}{c}\text { Unconfined } \\ \text { compression }\end{array}} \quad \frac{\begin{array}{c}\text { Purpose of } \\ \text { Material }\end{array}}{\text { Stabilizer }}$

$5.0 \%$

$4.7 \%$

2:1:3 (a sphalt, gasoline, water)

Rate of Material

$5 \%$

Cost

Not given

Effective

Strength

Increase

See comments Excellent
Not given

Not given

Not given

Not given

Mixing

Capability

Good

Total Material Cost

Per Cu Ft

of Treated Soil

Not given

Test Agency

Test Report

MIT

Effectiveness

Comments:

Samples treated with above materials were not compared to untreated samples. Tests we re conducted after a 24 hour humid cure followed by a 24 hour water immersion. Strength of the se samples was 165 psi.

* Basic material 
Category*

Asphalt

Basic Material

Straight run (40 to

50 pen) asphalt

Secondary Material

Phosphorus pentoxide

Antistripping additives

Water

Materia 1 Form*
Rate of Material

5 and $10 \%$

(cutback composition -

2:1 asphalt to gasoline
Cost

Not given

\begin{tabular}{cc}
0.5 to $3 \%$ & Not given \\
0.1 to $3 \%$ & Not given \\
$14.2 \%$ & Mixing \\
Type of Soil Treated & Capability \\
\hline Sandy soil & Good
\end{tabular}

$\frac{\text { Type of Test }}{\begin{array}{c}\text { Unconfined } \\ \text { compression }\end{array}} \quad \frac{\begin{array}{c}\text { Purpose of } \\ \text { Material }\end{array}}{\text { Stabilizer }}$

Effective

Strength

Increase

Effectiveness

See conments Excellent

Total Material Cost

Per Cu Ft

of Treated Soil

Test Agency

Test Report

Not given

MIT

Reference 36

Comments:

1. Phosphorus pentoxide $\left(\mathrm{P}_{2} \mathrm{O}_{5}\right)$ and antistripping additives were used separately and in combination at the percentage ranges cited above with cutback asphalt at the two rates shown. The antistripping agents were Al2D (lauryl amine) and Al8DA, Armeen 18D acetate (octodecyl amine acetate). The samples treated with $\mathrm{P}_{2} \mathrm{O}_{5}$ and other additives with asphalt were compared to samples treated with asphalt only. The semples were cured for 14 cays and after 7 days of water immersion, they were subjected to unconfined compression tests.

(Continued on next page)

* Basic material 
The results indicated that the 5 percent rate of asphalt in combination with $\mathrm{P}_{2} \mathrm{O}_{5}$ ( 1.5 percent). gave an increase in compressive strength of 60 percent over the asphalt only treated soil. The combination of $\mathrm{Al2D}$ ( 0.1 percent) and $\mathrm{P}_{2} \mathrm{O}_{5}$ ( 0.5 percent) gave the best results (48 percent increase over asphalt-treated soil).

At the 10 percent asphalt rate in combination with $\mathrm{P}_{2} \mathrm{O}_{5}$, an increase of 75 percent over the asphalt only treated soil resulted. The combination of $\mathrm{Al2D}$ ( 0.3 percent) and $\mathrm{P}_{2} \mathrm{O}_{5}$ (3.0 percent) gave the next best increase ( 54 percent).

Effectiveness: $\mathrm{P}_{2} \mathrm{O}_{5}$ is considered as the most effective additive with the basic material' on sandy silt soil.

2. Phosphorus pentoxide $\left(\mathrm{P}_{2} \mathrm{O}_{5}\right)$ was used separately and in combination with antistripping additives (Al2D - 0.1 to 0.3 percent and Al8DA 0.1 percent) and straight run asphalt ( 5 and 10 percent rates) on the following additional soils. Compressive tests were conducted after 14 days dry cure and 7 days water immersion.

a. Clayey silt: Mixing water content - 11 percent; asphalt cutback composition - 2:1 asphalt to gasoline.

The $\mathrm{P}_{2} \mathrm{O}_{5}$ ( 1.5 percent rate) with 5 percent rate asphalt gave the best results relative to the asphalt only treated samples, an increase of 93 percent in compressive strength.

The $\mathrm{P}_{2} \mathrm{O}_{5}$ ( 1.5 percent rate) with 10 percent rate asphalt gave the best results relative to the asphalt only treated samples, an increase of 166 percent in compressive strength.

b. Sandy clay: Mixing water content - 16 percent; asphalt cutback composition - 2:1 asphalt to gasoline.

The Al2D ( 0.2 percent) with 5 percent rate asphalt gave best results relative to asphalt only treated samples, an increase of 109 percent in compressive strength ( 23 psi asphalt only to 0.2 percent Al2D additive - $48 \mathrm{psi}$ ).

The $\mathrm{P}_{2} \mathrm{O}_{5}$ (3 percent rate) with 10 percent rate asphalt gave the best results relative to the asphalt treated samples, an increase of 560 percent in compressive strength:

c. Vicksburg loess: Mixing water content - 18.1 percent. Asphalt cutback composition - 2:1 asphalt to gasoline. 
At the 5 percent asphalt rate, no favorable results were achieved with the additives.

Asphalt rate - 10 percent. The $\mathrm{P}_{2} \mathrm{O}_{5}$ ( 3 percent) and $\mathrm{Al2D}$ ( 0.3 percent) gave the best results relative to the asphalt only treated samples, an increase of 1090 percent in compressive strength. $\mathrm{P}_{2} \mathrm{O}_{5}$ ( 3 percent) gave an increase of 570 percent in compressive strength.

d. Vicksburg buckshot: Mixing water content - 22.7 percent. Asphalt cutback composition - 2:1 asphalt to gasoline.

At the 5 percent asphalt rate, no favorable results were achieved with the additives.

At the 10 percent asphalt rate, no favorable results were achieved with the additives. 
Category*

Asphalt

Basic Material

Rate of Material

Cost

Straight run asphalt

$5 \%$

Not given

(100-200 pen)

Secondary Material

Emulsifying agents:

Duomeen $\mathrm{T}$

$5.0 \%$

4. $7 \%$

Hydrochloric acid

Solvent - ga soline

2:1:3 (asphalt, gasoline, water)

Additive - chromic

$0.25 \%$

Not given

Not given

chloride

$\frac{\text { Material Form * }}{\text { Liquid }}$

Type of Soil Treated

Clayey silt

Not given

Mixing

Capability

Good

\begin{tabular}{llll} 
Type of Test & $\begin{array}{l}\text { Purpose of } \\
\text { Material }\end{array}$ & $\begin{array}{l}\text { Effective } \\
\text { Strength } \\
\text { Increase }\end{array}$ & Effectiveness \\
\cline { 2 - 2 } $\begin{array}{c}\text { Unconfined } \\
\text { compression }\end{array}$ & Stabilizer & See comments & Excellent
\end{tabular}

Total Material Cost

Per Cu Ft

of Treated Soil

Not given

Test Agency

MIT
Test Report

Reference 40

Comments:

Samples treated with ab ove materials were not compared to untreated samples. Tests were conducted after a 24 hour humid cure plus a 24 hour water immersion.

Effectiveness: The above combination of materials produced samples with insignificant strengths.

Other samples contained the above materials plus 1.5 percent phosphoric acid, and this conbination was effective as a soil stabilizer (190 psi strength).

* Basic material 
Category*

\section{Asphalt}

Basic Material

Straight run asphalt (100-120 pen)
Rate of Material

$5 \%$
Cost

Not given

Secondary Material

\section{Emulsifying agents:}

Duomeen $T$

Hydrochloric acid
$5.0 \%$

$4.7 \%$

$0.1 \%$

Additive - chromic chloride

Water

Phosphoric acid

Material Form*

Liquid
$1.5 \%$

Type of Soil Treated

Clayey silt
Not given

Not given

Not given

Not given

Mixing

Capability

Good

\begin{tabular}{|c|c|c|c|}
\hline & Purpose of & $\begin{array}{l}\text { Effective } \\
\text { Strength }\end{array}$ & \\
\hline Type of Test & Material & Increase & Effectiveness \\
\hline $\begin{array}{l}\text { Unconfined } \\
\text { compression }\end{array}$ & Stabilizer & See comments & Excellent \\
\hline
\end{tabular}

Total Material Cost

Per Cu Ft

$\frac{\text { of Treated Soil }}{\text { Not given }}$

Test Agency

Test Report

MIT

Reference 40

Comments:

Samples treated with above materials we re not compared to untreated samples. Tests were conducted after a 24 hour humid cure followed by a 24 hour water immersion.

The strength of the treated samples was $110 \mathrm{psi}$.

* Basic material 
Category*

Asphalt

Basic Material

Straight run asphalt (100-200 pen)

Secondary Material

Emulsifying agents:

Duomeen $T$

$5.0 \%$

$4.7 \%$

Hydrochloric acid

Solvent - gasoline

Additive - chromic

Phosphoric acid

2:1:2 (asphalt, gasoline, water)

chloride

$0.1 \%$

1. $5 \%$

Material Form*

Liquid

Type of Soil Treated

Clayey silt

Effective

Purpose of Material

Type of Test

Unconfined compression
Strength

Increase

See comments
Not given

Not given

Not given

Not given

Mixing

Capability

Good

Total Material Cost

Per Cu Ft

of Treated Soil

Not given

Test Agency

Test Report

MIT

Reference 40

\section{Comments:}

Sampies treated with above materials were not compared to untreated samples. Tests were conducted after a 24 hour humid cure followed by a 24 hour water immersion.

The strength of the treated samples was 125 psi.

* Basic material 
Category*

Asphalt

$\frac{\text { Basic Material }}{\text { Straight run asphalt }}$
(100-120 pen)
$\frac{\text { Secondary Material }}{\text { Emulsifying agents: }}$

Duomeen $T$

$\frac{\text { Rate of Material }}{3,4, \text { and } 5 \%}$

Cost

Not given

Hydrochloric acid

Solvent - gasoline

$5.0 \%$

$4.7 \%$

Not given

Not given

Additives: Ferric chloride

halt, gasoline, wate $r$ )

Phosphoric acid $\left(\mathrm{H}_{3} \mathrm{PO}_{4}\right) \quad 1.5,2$, and $5 \%$

Not given

Not given

Mixing

Material Form*

Type of Soil Treated

Liquid

Clayey silt

Capability

Good

\begin{tabular}{|c|c|c|c|}
\hline Tes & Purpose of & $\begin{array}{l}\text { Effective } \\
\text { Strength } \\
\text { Increase }\end{array}$ & \\
\hline $\begin{array}{l}\text { Unconfined } \\
\text { compression }\end{array}$ & $\frac{\text { Material }}{\text { Stabilizer }}$ & $\frac{\text { Increase }}{\text { See comments }}$ & $\begin{array}{l}\text { Effectiveness } \\
\text { Excellent }\end{array}$ \\
\hline
\end{tabular}

Total Material Cost

Per Cu Ft

of Treated Soil

Not given

Test Agency

MIT

$\frac{\text { Test Report }}{\text { Reference } 40}$

Comments:

Samples treated with above materials we re not compared to untreated samples. Tests were conducted after 24 hours humid cure plus a 24 hour water immersion.

(Continued on next page)

* Basic material 
Effectiveness: Combination of materials above where several rates are given, all give high strength (155 psi) and are considered effective as stabilizers; however, shown below are strengths in order of effectiveness:

Asphalt (\%)

4

4

5

3

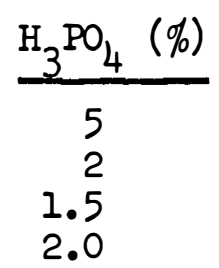

Strength (psi)

610

265

195

155 
Category*

Asphalt

Basic Material

Straight run asphalt

(100-120 pen)
Rate of Material

$5 \%$
Cost

Not given

Secondary Material

Emulsifying agents:

Duomeen $T$

$5 \%$

4. $7 \%$

Hydrochloric acid

Solvent - ga soline

Phosphoric acid $1.5 \%$
2:1:3 (asphalt, gasoline, water)

Type of Soil Treated

Clayey silt
Not given

Not given

Not given

Mixing

Capability

Good

Liquid

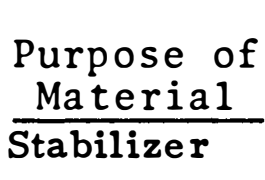

Effective

Strength

$\frac{\text { Type of Test }}{\text { Unconfined }}$

compression

Effectiveness

Excellent

Total Material Cost Per Cu Ft

of Treated Soil

Not given
Test Agency

MIT
Test Report

Reference 40

\section{Comments:}

Samples treated with above materials were not compared to untreated samples. Tests were conducted after a 24 hour humid cure followed by a 24 hour water immersion.

The strength of the treated samples was 125 psi.

* Basic material 
Category*

\section{Asphalt}

Basic Material

Rate of Material.

Cost

Straight run asphalt $5 \%$

Not given

(100-200 pen)

Secondary Material

Emulsifying agent:

Nonic 218

$6.25 \%$

Solvent - gasoline

Phosphoric acid $\left(\mathrm{H}_{3} \mathrm{PO}_{4}\right)$

2:1:3 (asphalt, gasoline, water) $1.5 \%$

Type of Soil Treated Clayey silt
Not given

Not given

Mixing Capability

Liquid

Purpose of

Type of Test

Unconfined

compression
Material

Stabilizer
Effective

Strength

Increase

See comments

Effectiveness

Good

Total Material Cost

Per Cu Ft

of Treated Soil

Test Agency

Test Report

Not given

MIT

Reference 40

Comments:

Samples treated with above materials were not compared to untreated samples. Tests were conducted after 24 hours humid cure plus 24 hours water immersion.

The addition of phosphoric acid is necessary for adquate stabilization given 24 hour humid cure plus 24 hour water immersion.

* Basic material 
Category*

Asphalt

$\frac{\text { Basic Material }}{\text { Straight run asphalt }}$
(100-120 pen)
$\frac{\text { Secondary Material }}{\text { Emulsifying agents: }}$

Duomeen $\mathrm{T} \quad 5.0 \%$

Hydrochloric acid

Solvent - ga soline

$\frac{\text { Rate of Material }}{5,10, \text { and } 12.5 \%}$

Cost

Not given

Additives - Ferric chloride

$4.7 \%$

Not given

Not given

Phosphoric acid

2:1:3 (asphalt, gasoline, water)

Not given

$0.1 \%$

$2.0 \%$

Material Form*

Liquid

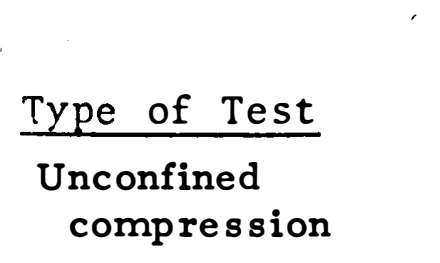

Type of Soil Treated

Clay (Vicksburg)
Mixing Capability

Good

Total Material Cost

Per Cu Ft

of Treated Soil

Not given
Effective

Strength

Increase

See comments
Effectiveness

Excellent

Comments:

Samples treated with above materials were not compared to untreatedsamples. Tests were conducted after a 24 hour humid cure followed by a 24 hour water immersion.

The most effective rate of asphalt was the 10 percent. The strength of samples treated with this asphalt and other materials was 85 psi. This value was substantially highe $r$ than values previously obtained with this soil using asphalt cutback-phosphoric acid combinations.

* Basic material 
Category: Cement

A6I 
Category*

Cement

Basic Material

Rate of Material

$5 \%$

Cost

Alumina cement

$5 \%$

Not given

Secondary Material

Modifiers (see comments) $\quad 1 \% \quad$ Not given

Material Form*

Type of Soil Treated

Mixing

Capability

Powder

Loess

Good

\begin{tabular}{|c|c|c|c|}
\hline Type of Test & $\begin{array}{l}\text { Purpose of } \\
\text { Material } \\
\end{array}$ & $\begin{array}{l}\text { Effective } \\
\text { Strength } \\
\text { Increase } \\
\end{array}$ & Effectiveness \\
\hline $\begin{array}{l}\text { Unconfined } \\
\text { Compression }\end{array}$ & Stabilizer & See comments & $\begin{array}{l}\text { Excellent } \\
\text { N/O Modifiers }\end{array}$ \\
\hline
\end{tabular}

Total Material Cost

Per Cu Ft

of Treated Soil

Not given

Comments:

Test Agency

WES
Test Report

Internal Data

(1956), not

published

Samples treated with cement and modifiers we re compared to untreated samples. Preparation of the samples was with the Harvard miniature compaction apparatus, five layers with an effort of 25 tamps per layer using a 40-1b spring tamper. Samples were cured in a humid room for 24 hours prior to te sting.

(Continued on next page)

* Basic material 
Modifiers:

Sodium hydroxide

Ammonium hydroxide

Calcium acrylate

Hydrated line

Portland cement
Polyvinyl alcohol

(grade 50-42)

Potassium permanganate

Potassium chloride

Sodium fluoride
Plaster of Paris

Ethyl silicate

Nitrobenzene

Sulphuric acid

phosphoric acid

Modifiers (continued):

Sodium tetraphosphate

Arquad $2 \mathrm{HT}$

Carboxymethyl cellulose

(grade 1800)

Chrome lignin

Glycerin

Effectiveness: Sodium hydroxide and ammonium hydroxide we re used separately with the basic material in an effort to alter the $\mathrm{pH}$ of the treated samples. There was no increase in strength.

The alumina cement alone met the requirements of Category 2 stabilization.

The only modifiers when used with the cement which exhibited any significant advantage were: Polyvinyl alcohol (72\%), carboxymethyl cellulose (69\%), and carboxymethyl cellulose (one part) plus (one part) hydrated lime (40\%). Numbers in parentheses are the percent increase in strength over cement only treated samples. 
Category*

Cement

Basic Material

Cement
Rate of Material

$10 \%$
Cost

Not given

Secondary Material

Additive s:

Sodium hydroxide plus 1:0, 2:1, 1:1, 11, 1:2, 0:1 Not given sodium sulfate

Material Form*

Type of Soil Treated Mixing

Powder

Clay (Texas \#2)

Capability

Good

$\begin{array}{llll}\text { Type of Test } & \begin{array}{c}\text { Purpose of } \\ \text { Material }\end{array} & \begin{array}{l}\text { Effective } \\ \text { Strength } \\ \text { Increase }\end{array} & \text { Effectiveness } \\ \begin{array}{c}\text { Unconfined } \\ \text { compression }\end{array} & \text { Stabilizer } & \text { See comments } & \text { Excellent }\end{array}$

Total Material Cost

Per Cu Ft

of Treated Soil

Not given

Test Agency

MIT
Test Report

Reference 39

Comments:

Samples treated with additive and cement were compared to cementtreated samples. The cure time varied from 1 to 28 days. Prior to te sting, the samples were immersed in water for 24 hours.

Effectiveness: The ratio of 1:0 sodium hydroxide to sodium sulfate in combination with cement gave the only significant increase in strength over the samples with only cement. (64 percent after 1 day cure and 67 percent after 28 days cure).

* Basic material 
Category*

Cement

Basic Material

Rate of Material

Cost

Cement

$3.5,6.8$, and $10 \%$

Not given

Secondary Material

Sodium hydroxide $(\mathrm{NaOH}) \quad 0.5 \mathrm{~N}$ and $1.0 \mathrm{~N} \quad$ Not given

Material Form*

Powder
Type of Soil Treated

Clay (Vicksburg)
Mixing

Capability

Good

\begin{tabular}{|c|c|c|c|}
\hline Type of Test & $\begin{array}{c}\text { Purpose of } \\
\text { Material }\end{array}$ & $\begin{array}{l}\text { Effective } \\
\text { Strength } \\
\text { Increase }\end{array}$ & Effectiveness \\
\hline $\begin{array}{l}\text { Unconfined } \\
\text { compression }\end{array}$ & $\overline{\text { Stabilizer }}$ & $\overline{\text { See comments }}$ & Excellent \\
\hline
\end{tabular}

Total Material Cost

Per Cu Ft

$\frac{\text { of Treated Soil }}{\text { Not given }} \frac{\text { Test Agency }}{\text { MIT }} \frac{\text { Test Report }}{\text { Reference } 40}$

\section{Comments:}

In the range of 3 to 10 percent cement, wet strength increased with the amount of cement and $1 \mathrm{~N} \mathrm{NaOH}$ giving the higher increase of strength after the one day cure; however, as the length of curing time increased, the difference in using $1 \mathrm{~N} \mathrm{NaOH}$ and $0.5 \mathrm{~N} \mathrm{NaOH}$ is insignificant.

To achieve a wet strength of 150 and 300 psi after 7 days of cure, 4 and 6 percent cement with $0.5 \mathrm{~N} \mathrm{NaOH}$ is needed, respectively.

* Basic material 
Category*

Cement

Basic Material

Rate of Material

Cost

Cement

$10 \%$

Not given

Secondary Material

Additive s:

Sodium hydroxide plus 1:0, 2:1, 1:1, 1:2, 0:1 sodium sulfate

Material Form*

Type of Soil Treated

Not given

Mixing

Powder

Sand (Wisconsin \#1)

Capability

Good

\begin{tabular}{|c|c|c|c|}
\hline Type of Test & $\begin{array}{c}\text { Purpose of } \\
\text { Material }\end{array}$ & $\begin{array}{l}\text { Effective } \\
\text { Strength } \\
\text { Increase }\end{array}$ & Effectiveness \\
\hline $\begin{array}{l}\text { Unconfined } \\
\text { compression }\end{array}$ & Stabilizer & See comments & Excellent \\
\hline
\end{tabular}

Total Material Cost

Per Cu Ft

of Treated Soil

Not given

Test Agency

MIT
Test Report

Reference 39

\section{Comments:}

Samples treated with additives and cement were compared to cementtreated samples. The cure time ranged for 1 to 28 days. Prior to testing, the samples were immersed in water for 24 hours.

Effectivene s8: The ratio of 1:0 sodium hydroxide to sodium sulfate in combination with cement gave less strength than the cement only treated samples. As the ratio of sodium hydroxide to sodium sulfate decreased, the effectiveness of the combined additive increased. The most effective combination of sodium hydroxide to sodium sulfate was $0: 1$ with the strength increase after 1 day cure being 720 percent and after 28 days cure being 1748 percent.

* Basic material 
Category*

Cement

Basic Material

Rate of Material

Cost

Cement $5 \%$

Not given

Secondary Material

\section{Additive s:}

Sodium hydroxide plus 1:0, 2:1, 1:1, 1:2, 0:2 sodium sulfate

Material Form*

Type of Soil Treated

Not given

Mixing

Capability

Powder

Silt

Good

\begin{tabular}{|c|c|c|c|}
\hline Type of Test & $\begin{array}{c}\text { Purpose of } \\
\text { Material } \\
\end{array}$ & $\begin{array}{l}\text { Effective } \\
\text { Strength } \\
\text { Increase } \\
\end{array}$ & Effectiveness \\
\hline $\begin{array}{l}\text { unconfined } \\
\text { compression }\end{array}$ & Stabilizer & See comments & Excellent \\
\hline
\end{tabular}

Total Material Cost

Per Cu Ft

of Treated Soil

Not given

Test Agency

MIT
Test Report

Reference 39

Comments:

Samples treated with additives and cement were compared to comenttreated samples. The cure time ranged from 1 to 28 days. Prior to testing, the samples were immersed in-water for 24 hours.

Effectiveness: The most effective ratio of sodium hydroxide to sodium sulfate was 1:1. The strength increase was 202 percent after 1-day cure and 292 percent after 28 days cure. However, all samples with the additives, regardless of the ratio of the two, were stronger than those treated with cement only.

* Basic material 
Category*

Cement

Basic Material Rate of Material

Cement (plus IN NaOH - $5 \%$

sodium hydroxide)

Secondary Material

See comments

Material Form*

Type of Soil Treated

Powder

Clay (Vicksburg)

Mixing

Capability

Good

\begin{tabular}{|c|c|c|c|}
\hline Type of Test & $\begin{array}{c}\text { Purpose of } \\
\text { Material }\end{array}$ & $\begin{array}{l}\text { Effective } \\
\text { Strength } \\
\text { Increase }\end{array}$ & Effectiveness \\
\hline $\begin{array}{l}\text { Unconfined } \\
\text { compression }\end{array}$ & Stabilizer & None & Excellent \\
\hline
\end{tabular}

Total Material Cost

Per Cu Ft

$\frac{\text { of Treated Soil }}{\text { Not given }} \quad \frac{\text { Test Agency }}{\text { MIT }} \frac{\text { Test Report }}{\text { Reference } 40}$

\section{Comments:}

Samples treated with $1 \mathrm{~N} \mathrm{NaOH}$ and cement were compared to samples treated only with cement. Tests were conducted after 1,7 , and 28 days humid cure plus 24 hours water immersion. The samples with the sodium hydroxide and cement for 1,7 , and 28 days cure had strength increases of 180,46 , and 41 percent, respectively, over samples treated with cement only.

Other individual additives te sted with cement plus $\mathrm{IN} \mathrm{NaOH}$ were:

Rosinamine' $D$ acetate $-0.025,0.1,0.2$, and 0.7 percent Melamine -1.0 percent

(Continued on next page) 
Aniline - 1.0 percent

Zinc nitrate -0.5 and 1.0 percent

Stannous chloride -0.1 percent

Ferric chloride -0.1 percent

Ferrous chloride -0.5 and 1.0 percent

None of the additives above produced any significant strength increase over that achieved with only cement plus sodium hydroxide (1N NaOH). 
Ca tegory*

Cement

Basic Material

Rate of Material

Cost

Fast Fix

$3,5,6,7,10,15$, and $20 \%$

$\$ 0.035$ per $1 \mathrm{~b}$

Secondary Material

Material Form*

Powder
Type of Soil Treated

Lean clay, heavy clay, and sand
Mixing Capability

Good
Effective

Purpose of Strength

$\frac{\text { Type of Test }}{\text { Unconfined }}$

compression
Increase

See comments

$\frac{\text { Effectiveness }}{\text { See comments }}$

Total Material Cost

Per $\mathrm{Cu} \mathrm{Ft}$

$\frac{\text { of Treated Soil }}{\text { Not given }} \quad \frac{\text { Test Agency }}{\text { WES }}$

Test Report

(1971), not

published

Comments:

Samples treated with Fast Fix were compared to samples treated with Type I portland cement. Samples were prepared with a Harvard miniature compaction apparatus, five layers, ten tamps per layer of a 40-1b spring tamper. Prior to tests as a Category 2 stabilizer, the samples were cured at 100 percent relative humidity followed by 24 hours water immersion.

Effectiveness: To satisfy the Category 2 stabilization, approximately 15 percent and more than 15 percent $F$ ast $F$ ix is required on lean and heavy clay, respectively. Approximately 7.5 percent is required on sand.

(Continued on next page)

Basic material 
To satisfy the same requirements on all three soils, only approximately 6 percent portland cement is required. Cement also costs less than one third that of Fast Fix. From the se two standpoints, the Fast Fix does not offer any advantages in stabilization. 
Category*

Cement

Basic Material

Rate of Material

Cost

Lumnite cement

5,10 , and $15 \%$

Not given

Secondary Material

Material Form*

Powder
Type of Soil Treated

Sand, loess, and heavy clay
Mixing

Capability

Good
Effective

Purpose of Strength

Type of Test

Unconfined compression
Increase

See comments
Effectiveness

Excellent

Total'Material Cost

Per Cu Ft

\begin{tabular}{|c|c|c|}
\hline Not given & WES & $\begin{array}{l}\text { Internal Dat } \\
(1956) \text {, not } \\
\text { published }\end{array}$ \\
\hline
\end{tabular}

Comments:

Samples treated with lumnite cement were compared with those treated with portland cement. Samples were prepared using the Harvard miniature compaction apparatus. For the loess and heavy clay samples, compaction was applied on each of three layers by 25 tamps of a 40-1b spring tamper. The sand samples were compacted on each of three layers by 25 tamps of a 20-1b spring tamper. Cure times were 6 hours, 24 hours, 3 days, and 7 days under humid conditions prior to testing.

Effectiveness: The rate of strength development and ultimate strengths achieved in the loess and heavy clay using the lumnite cement are less (Continued on next page)

Basic material 
than that achieved using normal portland cement under comparable test conditions.

On sand, the lumnite cement was much more effective than portland cement. At the 10 percent rate of treatment, the strength increase of the lumnite over the portland cement was 429,131 , and 83 percent after 1,3 , and 7 days cure, respectively. Higher strength values were achieved with 15 percent lumnite cement. 
Category*

Cement

Basic Material

Rate of Material

Cost

Plaster of Paris

3, 5, and $10 \%$

Not given

Secondary Material

Material Form*

Powder
Type of Soil Treated

Lean clay and heavy clay
Mixing

Capability

Good

\begin{tabular}{|c|c|c|c|}
\hline Type of Test & $\begin{array}{c}\text { Purpose of } \\
\text { Material }\end{array}$ & $\begin{array}{l}\text { Effective } \\
\text { Strength } \\
\text { Increase }\end{array}$ & Effectiveness \\
\hline $\begin{array}{l}\text { Unconfined } \\
\text { compression }\end{array}$ & Stabilizer & See comments & None \\
\hline
\end{tabular}

Total Material Cost

Per Cu Ft

of Treated Soil

Not given

Comments:

Test Agency

WES
Test Report

Internal data (1956-57), not

Published

Treated samples were compared to untreated samples. Preparation of the samples was with a Harvard miniature compaction apparatus, five layers, ten tamps per layer with a 20-1b spring tamper. The samples were te sted against Category 1 stabilization requirements.

Effectiveness: The strength increase of the treated samples as compared to the untreated varied 200 to 1700 percent; however, this did not satisfy the requirements.

* Basic material 
Category*

Cement

Basic Material

Portland cement

Rate of Material

Cost

Not given

Secondary Material

Material Form*

Powder
Type of Soil Treated

Loe 88

Effective

Strength

Increase

$239 \%$

Stabilizer

Waterproofer
Purpose of

Material
Mixing

Capability

Good $\frac{\text { Type of Test }}{\text { Unconfined }}$

compression

Total Material Cost

Per Cu Ft

of Treated Soil

Not given

$\frac{\text { Test Agency }}{\text { WES }}$

$\frac{\text { Effectiveness }}{\text { Excellent }}$

Comments:

Treated samples were compared to untreated samples ( 23 psi unconfined compression strength). Samples prior to tests were airdried 4 days followed by 4 days wetting by permeation. The strength of the treated samples was $78 \mathrm{psi}$ which was in increase of 239 percent. The material showed promise as a waterproofer.

This material was also subjected to field investigations at WES as a dustproofer and waterproofer; however, the result did not indicate the need for additional tests of this material.

* Basic material 
Category*

Cement

Basic Material

Portland cement

Rate of Material

Secondary Material

Material Form*

Powder
Type of Soil Treated

Lean clay clay

$5 \%$
Cost

Not given 
Category*

Cement

Basic Material

Portland Cement
Rate of Material $5 \%$
Cost

Not given

Secondary Material

Additives (see coments) $\quad 0.5$ and $1.0 \% \quad$ Not given

Material Form*

Type of Soil Treated

Mixing

Powder

Clayey Silt

Capability

Good

Effectiveness

Type of Test

Purpose of

Effective

Material

Strength

Unconfined

Stabilizer

Increase

See comments

Excellent

compression

Total Material Cost

Per Cu Ft

of Treated Soil

$\frac{\text { Test Agency }}{\text { MIT }}$

$\frac{\text { Test Report }}{\text { Reference } 35}$

\section{Comments:}

The treated samples with additives were compared to soil-cement samples. Compressive strengths were determined after 7 and 28 days of soaking. The soil-cement samples after 7 days soak had a compressive strength of 170 psi and 28- psi after 28 days soak in water.

(Continued on next page).

* Basic material 


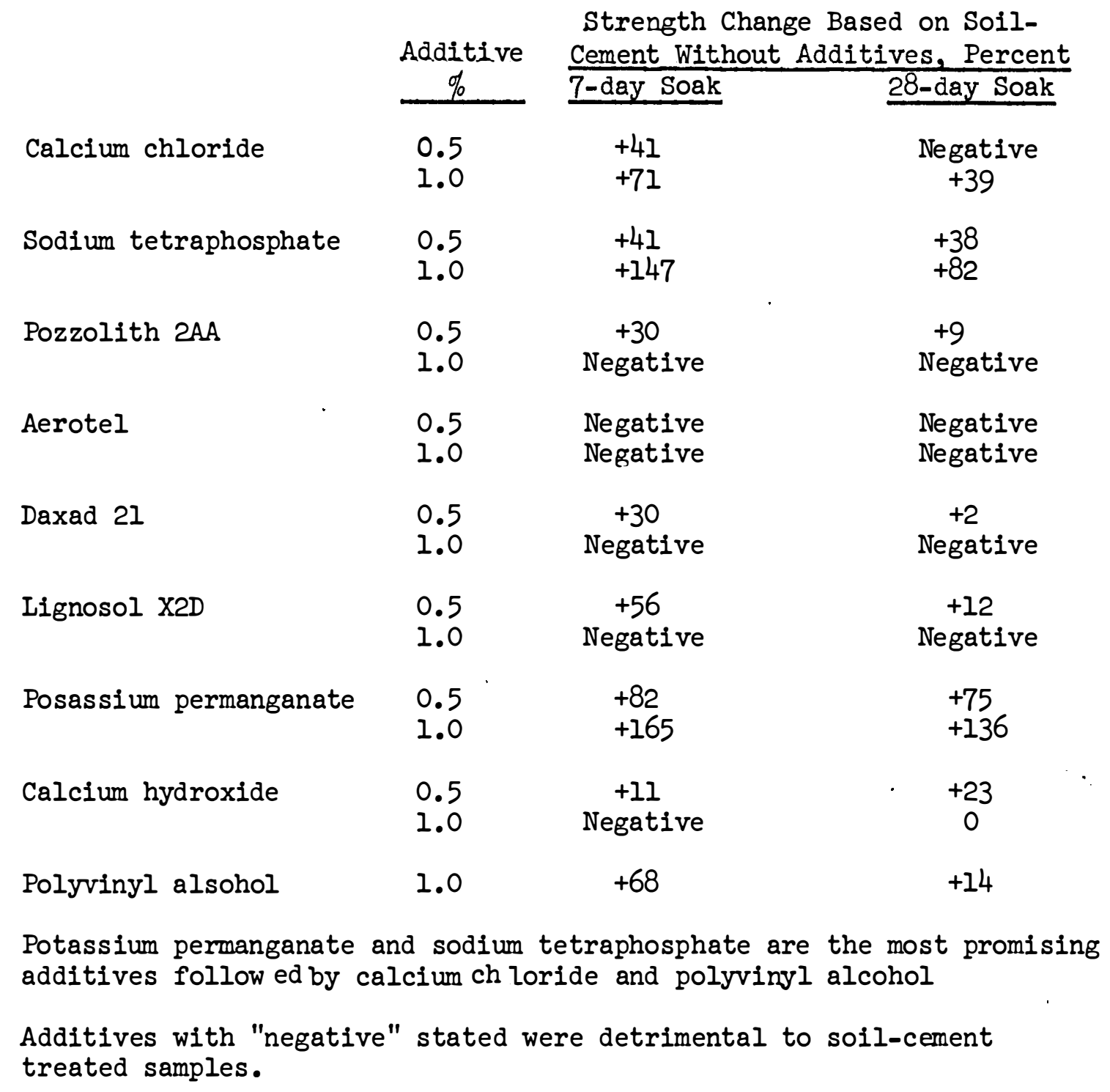


Category*

Cement

Basic Material

Portland cement $\frac{\text { Rate of Material }}{5 \%}$
Cost

Not given

Secondary Material

\begin{tabular}{ccc}
\hline $\begin{array}{c}\text { Arquad 2HT plus sodium } \\
\text { hydroxide }\end{array}$ & 0.1 plus $0.99 \% ;$ & Not given \\
& 0.5 plus $1.08 \% ;$ and & \\
Arquad 12 & 1.0 plus $1.07 \%$ & Not given
\end{tabular}

Material Form*

Powder

Type of Soil Treated

Clay (Texas \#2)

Effective

$\frac{\text { Type of Test }}{\text { Unconfined }}$ compression
Purpose of

Strength

Increase

Material

Stabilizer
Mixing

Capability

Good

Effectiveness

Excellent

Total Material Cost

Per Cu Ft

of Treated Soil

Not given

Test Agency

MIT
Test Report

Reference 39

Comments:

Arquad $2 \mathrm{HT}$ - di-hydrogenated tallow dimethyl ammonium chloride

Arquad 12 - lauryl trimethyl ammonium chloride

Samples treated with additives and cement compared to samples treated with cement only. After cure time shown below and prior to tests, samples were immersed in water for 24 hours.

(Continued on next page)

* Basic material 
Additive

Additive
None
Arquad 2HT plus
sodium hydroxide

Arquad 12
Strength

Change

Based

on Soil

Without

Additive

$\%$ Curing Strength

Additive

Days

psi

172

218

180

180

0.1

0.00

100

200

250

390

208

291

372

423
Negative
Negative
$+79$
$+117$

$\begin{array}{lrll}0.5 & 1 & 208 & +21 \\ 1.08 & 4 & 291 & +34 \\ & 7 & 372 & +107 \\ & 28 & 423 & +135\end{array}$

$\begin{array}{ll}1.0 & 7 \\ 1.07 & 4 \\ & 28\end{array}$

293

280

365

$+70$

$+28$

$+102$

$+102$

1.0

$\begin{array}{rr}1 & 139 \\ 4 & 184 \\ 7 & 208 \\ 28 & 262\end{array}$

Negative

Negative $+16$

$+46$

Effectiveness: Arquad 2HT ( 0.1 percent) plus sodium hydroxide ( 0.99 percent) with cement produced the highest strength increast except for the one day cure. Arquad 2 HT ( 1 percent) plus sodium hydroxide ( 1.0 percent) gave the greatest increase, 70 versus 21 percent for the first rates given. The remaining materials only gave strength increase after 7 and $\cdot 28$ days cure. 
Category*

Cement

Basic Material

Rate of Material

Cost

Portland cement

$5 \%$

Not given

Secondary Material

See comments for additives

Material Form*

Powder
Type of Soil Treated

Clay (Vicksburg)
Mixing

Capability

Good

\begin{tabular}{|c|c|c|c|}
\hline Type of Test & $\begin{array}{l}\text { Purpose of } \\
\text { Material }\end{array}$ & $\begin{array}{l}\text { Effective } \\
\text { Strength } \\
\text { Increase }\end{array}$ & Effectiveness \\
\hline $\begin{array}{l}\text { Unconfined } \\
\text { compression }\end{array}$ & Stabilizer & See comments & Excellent \\
\hline
\end{tabular}

Total Material Cost

Per Cu Ft

of Treated Soil

Not given $\frac{\text { Test Agency }}{\text { MIT }}$
Test Report

Reference 39

\section{Comments:}

The samples treated with cement plus each additive were compared to samples treated with only cement. The samples were cured for 1 , 4,7 , and 28 days plus 24 hours water immersion and then subjected to tests.

The additives with rates (percent) are shown below:

a. Sodium hydroxide -0.48 and 1.00 percent

b. Ferric shloride -0.10 and 0.5 percent

plus sodium hydroxide -1.03 and 1.00 percent

(Continued on next page)

Basic materia1 
c. Arquad $2 \mathrm{HT}$ (di-hydrogenated tallow dimethyl ammonium chloride) -0.10 and 0.20 percent

d. Arquad 12 (laurly trimethyl ammonium chloride) -0.50 and 1.00 percent plus sodium hydroxide -0.98 and 0.96 percent

e. Triethylene tetramine (TTA) -0.50 and 1.00 percent plus sodium hydroxide -0.96 and 0.98 percent

f. Octylamine (soil pretreated with this material prior to the addition of sodium hydroxide ) -0.50 and 1.00 percent plus sodium - 1.04 and 1.00 percent

Effectiveness: All additives with cement gave some increase in strength over only cement-treated samples. Sodium hydroxide (1. 00 percent) was the most effective additive and gave the greatest strength increase for all cure days. However, 10 percent cement only treated samples gave better results than 5 percent cement plus the sodium hydroxide. 
Category*

Cement

Basic Material

Rate of Material

Cost

Portland cement

5 and $10 \%$

Not given

Secondary Material

Chemical additives (see Varied (0.5 to 2.0\%) Not given comments)

Material Form*

Powder
Type of Soil Treated

Silt
Mixing

Capability

Good

\begin{tabular}{|c|c|c|c|}
\hline Type of Test & $\begin{array}{c}\text { Purpose of } \\
\text { Material }\end{array}$ & $\begin{array}{l}\text { Effective } \\
\text { Strength } \\
\text { Increase }\end{array}$ & Effectiveness \\
\hline $\begin{array}{l}\overline{\text { Unconfined }} \\
\text { compression }\end{array}$ & $\overline{\text { Stabilizer }}$ & See comments & $\overline{\text { Excellent }}$ \\
\hline
\end{tabular}

Total Material Cost

Per Cu Ft

$\frac{\text { of Treated Soil }}{\text { Not given }} . \frac{\text { Test Agency }}{\text { MIT }} \frac{\text { Test Report }}{\text { Reference } 37}$

Comments:

The molding moisture content varied from 20.1 to 21.7 percent. The number of curing hours varied from 4 to 168. In the tabulation below, data are given on the rates (percent) and hours that gave the most effective combination with the materials used. The Optimum rate of additive is also given. The cure condition for the optimum rate of additive is also given. The cure conditions for the samples were as follows: room temperature, 100 percent relative humidity, 24 hours immersion in water, and then samples subjected to tests.

(Continued on next page)

* Basic material 


\begin{tabular}{|c|c|c|c|c|c|}
\hline Additive & $\begin{array}{c}\text { Concentration } \\
\text { of Additive } \\
\%\end{array}$ & $\begin{array}{c}\text { Molding } \\
\text { Water } \\
\text { Content } \\
\%\end{array}$ & $\begin{array}{l}\text { Curing } \\
\text { Time } \\
\text { Hrs } \\
\end{array}$ & $\begin{array}{c}\text { Compressive } \\
\text { Strength } \\
\text { psi }\end{array}$ & $\begin{array}{c}\text { Strength } \\
\text { Change } \\
\text { Based On } \\
\text { Soil Cement } \\
\text { Without } \\
\text { Additive } \\
\% \\
\end{array}$ \\
\hline \multicolumn{6}{|l|}{ A. 5 percent cement } \\
\hline None & 0 & 20.9 & 168 & 107 & -- \\
\hline Sodium metasilicate & 1.0 & 20.6 & 168 & 359 & +236 \\
\hline Sodium silicate & 1.0 & 20.5 & 168 & 277 & +159 \\
\hline Sodium hydroxide & 1.0 & 20.5 & 168 & 285 & +166 \\
\hline Potassium hydroxide & 1.43 & 21.0 & 168 & 270 & +153 \\
\hline Lithium hydroxide & 0.59 & 20.8 & 168 & 198 & +85 \\
\hline Sodium sulfite & 1.0 & 21.2 & 168 & 322 & +200 \\
\hline Sodium carbonate & 1.0 & 20.5 & 168 & 375 & +250 \\
\hline Sodium bicarbonate & 1.0 & 21.0 & 168 & 248 & +132 \\
\hline \multicolumn{6}{|l|}{ B. 10 percent cement } \\
\hline None & 0 & 19.6 & 168 & 312 & - \\
\hline Sodium metasilicate & 1.0 & 19.1 & 168 & 515 & +65 \\
\hline Sodium hydroxide & 1.0 & 19.2 & 168 & 462 & +48 \\
\hline Sodium carbonate & 1.0 & 19.3 & 168 & 492 & +58 \\
\hline
\end{tabular}

\section{Effectiveness:}

5 percent cement. The additive, sodium carbonate, gave the most effective increase in compressive strength over the soil-cement samples. Sodium metasilicate and sodium sulfite were next in order of effectiveness. However, all chemical additives were effective in increasing the sample strength over the cement only treated samples.

10 percent cement. Sodium metasilicate was the most effective additive used with 10 percent cement. All additives, however, increased the compressive strength of the samples. The percent increase for the 10 percent cement was not as great an increase as for the 5 percent cement; however, the compressive strengths were higher when compared to the cement only treated samples. 
Category*

Cement

$\frac{\text { Basic Material }}{\text { Portland cement }} \quad \frac{\text { Rate of Material }}{10 \%}$

$\frac{\text { Cost }}{\text { Not given }}$

Secondary Material

Calcium shloride

Sodium hydroxide

Sodium carbonate

Sodium sulfite

$0.6 \%$

0.5 and $1.0 \%$

$1.0 \%$

$1.0 \%$

Sodium sulfate

$0.5 \%$

$1.05 \%$

Sodium metasilicate

Material Form*

Type of Soil Treated

Powder

Sand (Winconsin \#2)

Not given

Not given

Not given

Not given

Not given

Not given

Mixing

Capability

Good

Effective

Purpose of Strength

$\frac{\text { Type of Test }}{\text { Unconfined }}$

Material Increase

Stabilizer

See comments

Effectiveness

compression

Total Material Cost

Per Cu Ft

of Treated Soil

Test Agency

Test Report

Not given

MIT

Reference 38

Comments:

Samples treated with the secondary materials were compared to samples treated with cement only. Curing time was 1, 4, and 7 . days followed by 1 day of water immersion prior to tests. Each secondary material was used with 10 percent cement.

(Continued on next page)

* Basic material 
Effectiveness: All materials except 1.0 percent sodium hydroxide, one day cure time, increased the strength of the cement-treated samples for all cure days. Sodium metasilicate (l percent) was the most effective in that after one day cure the strength was increased over the cement-treated only by 734 percent and after 7 days cure the strength was increased by 95 percent. All materials accelerated the rate of cure of the samples. 
Category*

Cement

Basic Material

Rate of Material

Cost

Portland cement $5 \%$

Not given

Secondary Material

See comments

Material Form*

Powder
$1 \%$

Type of Soil Treated

Lean clay Heavy clay
Mixing

Capability

Good

Good

\begin{tabular}{|c|c|c|c|}
\hline Type of Test & $\begin{array}{l}\text { Purpose of } \\
\text { Material }\end{array}$ & $\begin{array}{l}\text { Effective } \\
\text { Strength } \\
\text { Increase }\end{array}$ & Effectiveness \\
\hline $\begin{array}{l}\text { Unconfined } \\
\text { compression }\end{array}$ & Stabilizer & See comments & Excellent \\
\hline
\end{tabular}

Total Material Cost

Per Cu Ft

of Treated Soil

Not given

$\frac{\text { Test Agency }}{\text { WES }}$
Test Report

Reference 18

\section{Comments:}

Samples were molded in a Harvard Miniature Compaction Apparatus in five layers (each layer compacted with ten tamps of a 40-1b spring. tamper). Samples were tested after 24 hours cure at 100 percent relative humidity and after a 24 hour cure at 100 percent relative humidity followed by 24 hours water immersion. The strength of the untreated soils was about 20 psi. Materials which when added to the soil helped to increase the strength from 20 to $100 \mathrm{psi}$ or greater were considered to have potential as stabilizers.

Portland cement (5\%) was used alone with both soils and in combination with the following materials on both soils. (Each material was (Continued on next page)

* Basic material 
used at a 1 percent rate.)

Sodium hydroxide, sodium sulfate, sodium aluminate, sodium orthosilicate, sodium metasilicate, sodium hydroxide plus sodium or thosilicate, sodium hydroxide plus sodium metasilicate, sodium sulfate plus sodium orthosilicate, and sodium sulfate plus sodium meta silicate.

Effectiveness: Lean clay - Samples treated with 5 percent portland cement with no additives gave the best results (185 psi after 24 hours dry cure and 150 psi after 24 hours soak.) Sodium orthosilicate and sodium metasilicate each with cement gave some what higher wet strengths; however, the dry strengths were less than that for cement only treated samples.

Heavy clay - Samples treated with 5 percent portland cement and 1 percent sodium hydroxide gave the be st results (165 psi dry strength and 150 psi after 24 hours soak). Treatment with only 5 percent portland cement was the next best treatment (145 psi dry strength and 106 psi after soak). 
Category*

Cement

Basic Material

Rate of Material

Cost

Portland cement

$5 \%$

Not given

Secondary Material

Dispersants (see

comments)

See comments

Material Form*

Type of Soil Treated

Mixing

Capability

Powder

Clayey silt

Good

\begin{tabular}{|c|c|c|c|}
\hline Type of Test & $\begin{array}{c}\text { Purpose of } \\
\text { Material }\end{array}$ & $\begin{array}{l}\text { Effective } \\
\text { Strength } \\
\text { Increase } \\
\end{array}$ & Effectiveness \\
\hline Unconfined & Stabilizer & See comments & Excellent \\
\hline
\end{tabular}

Total Material Cost

Per Cu Ft

of Treated Soil

Not given

Test Agency

Test Report

MIT

Reference 35

Comments:

The concentration of dispersants was 1 percent except for the Kent wetting agent which was 5 percent. The treated samples were compared to a soil cement sample with 270 psi compressive strength.

(Continued on next page)

* Basic material 
Dispersant

Lignosol X2D

Lignosol SF

Lignosol SFX

Pozzolith 2AA

Daxad 21

Kent wetting agent

Sodium thiosulfate

Calcium phosphate-monobasic

Sodium fluosilicate

Trisodium phosphate

Sodium tetraphosphate

Tetrasodium pyrophosphate

Modified sodium phosphate

Trisocium phosphate (anhydrous)

Sodium tripolyphosphate
Strength Change Based on Soil Cement, Percent

$+41$

$+11$

Negative

$+59$

$+33$

$+22$

$+52$

Negative

Negative

$+37$

$+19$

0

Negative

$+26$

$+7$

As seen from the data above, the most promising were pozzolith $2 \mathrm{AA}$, sodium thiosulfate, lignosol $\mathrm{X} 2 \mathrm{D}$, and trisodium phosphate. Others which indicated some improvement were Kent wetting agent, sodium tetraphosphate, Daxad 21, and trisodium phosphate (anhydrous).

The maximum compressive strength of soil-cement using 10 percent cement and without dispersant was $390 \mathrm{psi}$. The four most promising gave a strength increase approximating that of an additional 5 percent cement over the base amount of 5 percent (about same strength as 10 percent cement only). 
Category*

Cement

Basic Material

Portland cement
Rate of Material

$5 \%$
Cost

Not given

Secondary Material

Sodium hydroxide

Sodium carbonate

Sodium metasilicate

Material Form*

Powder

$$
\begin{aligned}
& 1.0 \% \\
& 1.0 \% \\
& 1.0 \%
\end{aligned}
$$

Type of Soil Treated

Loess
Not given

Not given

Notiliven

Capability

Good

$\frac{\text { Type of Test }}{\begin{array}{c}\text { Unconfined } \\ \text { compression }\end{array}} \quad \frac{\begin{array}{l}\text { Purpose of } \\ \text { Material }\end{array}}{\text { Stabilizer }} \quad \frac{\begin{array}{l}\text { Effective } \\ \text { Strength } \\ \text { Increase }\end{array}}{\text { See comments }} \quad \frac{\text { Effectiveness }}{\text { Excellent }}$

Total Material Cost

Per Cu Ft

$$
\text { of Treated Soil }
$$

Not given
Test Agency

MIT
Test Report.

Reference 38

\section{Comments:}

The treated samples (with each additive) were compared to samples treated only with 5 percent cement. Cure time is listed below; however, before testing, the samples-were also subfected- to 24 hours waterimmersion.

(Continued on next page)

* Basic material 
Additive

None

Sodium hydroxide

Sodium carbonate

Sodium metasilicate

$\begin{array}{cccc}\begin{array}{c}\% \\ \text { Additive }\end{array} & \begin{array}{c}\text { Curing } \\ \text { Days }\end{array} & \begin{array}{c}\text { Strength } \\ \text { psi }\end{array} \\ 0 & & 1 & 102 \\ & 4 & 175 \\ & 7 & & 132 \\ & 28 & 232\end{array}$

1.0

$\begin{array}{rr}1 & 98 \\ 4 & 274 \\ 7 & 355 \\ 28 & 450\end{array}$

1.0

$\begin{array}{rr}1 & 146 \\ 4 & 180 \\ 7 & 175 \\ 28 & 310\end{array}$

1.0
Strength Change

Based on Soil Without

Additives

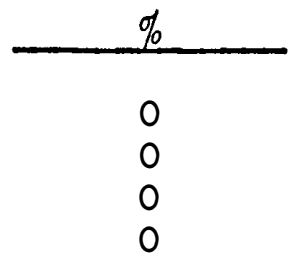

Negative

$+57$

$+169$

$+94$

$+43$

$+3$

$+33$

$+34$

$\begin{array}{rr}1 & 211 \\ 4 & 264 \\ 7 & 265 \\ 28 & 430\end{array}$

$+107$

$+51$

$+100$

$+86$

Effectiveness: Except for the slow curing after one day, sodium. hydroxide is the most effective in increasing the strength. Sodium metasilicate and sodium carbonate are next in order of effectiveness. 
Category*

Cement

Basic Material

Rate of Material

Portland cement

$10 \%$

Cost

Not given

Secondary Material

Sodium hydroxide

Sodium carbonate

0.25 to $1.0 \%$

Not given

$1.0 \%$

$1.0 \%$

Sodium metasilicate

0.54 and $1.08 \%$

Not given

Not given

Sodium sulfate

Not given

Material Form*

Powder
Type of Soil Treated

Sand (Wisconsin \#I)
Mixing Capability Good

\begin{tabular}{|c|c|c|c|}
\hline Type of Test & $\begin{array}{l}\text { Purpose of } \\
\text { Material }\end{array}$ & $\begin{array}{l}\text { Effective } \\
\text { Strength } \\
\text { Increase }\end{array}$ & Effectiveness \\
\hline $\begin{array}{l}\text { Unconfined } \\
\text { compression }\end{array}$ & Stabilizer & See comments & Excellent \\
\hline
\end{tabular}

Total Material Cost Per Cu Ft

$\frac{\text { of Treated Soil }}{\text { Not given }}$

Test Agency

MIT
Test Report

Reference 38

Comments:

Samples treated with the cement and additives were compared to samples treated only with cement. Tests were run on samples after $1,4,7$, and 28 days of cure followed by 24 hours water immersion.

(Continued on next page)

* Basic material 
Effectiveness: The following secondary materials gave no increase in strength of the cement-treated samples or the addition of these materials was detrimental to the strength of the samples: sodium hydroxide, sodium carbonate, and sodium metasilicate. Sodium sulfate was very effective in combination with 10 percent cement in improving the strength of treated samples. At0.54 percent sodium sulfate, the strength increased from 500 after one day cure to 1030 percent after 28 days cure over that for cement only treated samples. At 1.08 percent sodium sulfate, the strength increased from 720 after one day cure to 1739 percent after 28 days cure over that for cement only treated samples . 
Category*

Cement

Basic Material

$\frac{\text { Rate of Material }}{5 \%}$

Cost

Not given

Secondary Material

Sodium hydroxide, sodium carbonate, sodium metasilicate, sodium sulfate, sodium aluminate, sodium fluosilicate, sodium fluoride, sodium fluoborate, and sodium

tetraborate

$$
\begin{aligned}
& \text { All materials Not given } \\
& \text { were each tested } \\
& \text { with cement at } 0.5 \text {, } \\
& 1.0 \text {, and } 2.0 \% \text { rates }
\end{aligned}
$$

Material Form*

Powder
Type of Soil Treated silt

\begin{tabular}{|c|c|c|c|}
\hline Type of Test & $\begin{array}{l}\text { Purpose of } \\
\text { Material }\end{array}$ & $\begin{array}{l}\text { Effective } \\
\text { Strength } \\
\text { Increase }\end{array}$ & Effect iveness \\
\hline $\begin{array}{l}\text { Unconfined } \\
\text { compression }\end{array}$ & Stabilizer & See comments & Excellent \\
\hline
\end{tabular}

Mixing Capability Good

Total Material Cost Per $\mathrm{Cu} \mathrm{Ft}$

of Treated Soil

Not given

\author{
Test Agency \\ MIT
}

Test Report

Reference 38

Comments:

Treated samples (with each additive) were compared to samples treated only with 5 percent cement. Cure time is listed below; however, before testing, the samples were also subjected to 24 hours water immersion. of the three rates for eacin additive used, the most effective rate is shown below:

(Continued on next page)

* Basic material 
$\underline{\text { Additive }}$

None

Sodium hydroxide

Sodium carbonate

Sodium metasilicate

Sodium sulfate

Sodiuin aluminate
Strength Change

Based on Soil

Without

Additives

$\begin{array}{cccc}\begin{array}{c}\% \\ \text { Additive }\end{array} & \begin{array}{c}\text { Curing } \\ \text { Days }\end{array} & \begin{array}{c}\text { Strength } \\ \text { psi }\end{array} \\ 0 & & & 80 \\ & 4 & 90 \\ & 7 & 95 \\ & 28 & 125\end{array}$

1.0

$\begin{array}{rr}1 & 145 \\ 4 & 217 \\ 7 & 235 \\ 28 & 280\end{array}$

$+80$

$+141$

$+148$

$+124$

1.0

$\begin{array}{rr}1 & 140 \\ 4 & 188 \\ 7 & 220 \\ 28 & 285\end{array}$

$+75$

$+109$

$+132$

$+128$

$\begin{array}{rr}1 & 135 \\ 4 & 198 \\ 7 & 218 \\ 28 & 344\end{array}$

$+69$

$+120$

$+130$

$+175$

$+185$

$+205$

$+242$

$+248$

1.0

$\begin{array}{rr}1 & 228 \\ 4 & 275 \\ 7 & 325 \\ 28 & 435\end{array}$

$+188$

$+213$

$+247$

$+240$

Effectiveness: Other additives which were used (sodium fluosilicate, sodium fluoride, sodium fluoborate, ET-218, and sodium tetraborate) were either detrimental when added to the cement or no significant strength increase resulted.

Sodium aluminate ( 0.5 percent) and sodium sulfate ( 1.0 percent) were very effective in increasing the strength of the treated samples. Sodium hydroxide, carbonate, and metasilicate were also effective in increasing the strength of the additive-cement-treated samples over the strength of the cement only treated samples. 
Category*

Cement

Basic Material

Portland Cement

$\frac{\text { Rate of Material }}{10 \%}$

$\frac{\text { Cost }}{\text { Not given }}$

Secondary Material

Additives

Sodium hydroxide

Sodium sulfate

$0.98,1.93$, and $2.90 \%$

Not given

$1.71,3.32$, and $4.63 \%$

Not given

Sodium aluminate

$0.51,1.03$, and $2.08 \%$

Not given

Material Form*

Powder
Type of Soil Treated silt
Mixing

Capability

Good

\begin{tabular}{|c|c|c|c|}
\hline Type of Test & $\begin{array}{c}\text { Purpose of } \\
\text { Material } \\
\end{array}$ & $\begin{array}{l}\text { Effective } \\
\text { Strength } \\
\text { Increase }\end{array}$ & Effectiveness \\
\hline $\begin{array}{l}\text { Unconfined } \\
\text { compression }\end{array}$ & Stabilizer & See coments & Excellent \\
\hline
\end{tabular}

Total Material Cost

Per Cu Ft

of Treated Soil

Not given

$\frac{\text { Test Agency }}{\text { MIT }}$

$\frac{\text { Test Report }}{\text { Reference } 39}$

\section{Comments:}

Samples treated with cement plus each additive were compared to samples treated with cement only. ill samples were tested after the cure time shown below followed by 24 hours water immersion. The combinations (percent) of materials which gave best results are shown below:

(Continued on next page)

* Basic material 
Additive

None

Sodium hydroxide

Sodium sulfate $\frac{\text { Addit }}{0}$

$\%$

$\%$

\begin{tabular}{c}
$\begin{array}{c}\text { Curing } \\
\text { Days }\end{array} \begin{array}{c}\text { Strength } \\
\text { psi }\end{array}$ \\
\hline
\end{tabular}

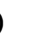

$\begin{array}{rr}1 & 128 \\ 4 & 208 \\ 7 & 283 \\ 28 & 360\end{array}$

0.98

$\begin{array}{rr}1 & 192 \\ 4 & 331 \\ 7 & 362 \\ 28 & 478\end{array}$

$3 \cdot 32$

$\begin{array}{rr}1 & 315 \\ 4 & 426 \\ 7 & 410 \\ 28 & 640\end{array}$

Strength Change

Based on Soil

Without

Additive
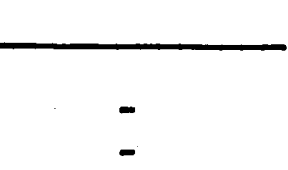

-

-

$+50$

$+59$

$+28$

$+33$

$+146$

$+105$

$+45$

$+78$

Effectiveness: The sodium sulfate ( 3.32 percent) was the most effective additive.

The amount of strength increase with additives and cement is less than that for 5 percent cement treatment; however, the early strength of the samples is much better. 
Category*

Cement

Basic Material

Portland cement

$\frac{\text { Rate of Material }}{5 \%}$

Cost

Not given

Secondary Material

Sodium hydroxide
Sodium sulfate
Socium aluminate
Ferric chloride plus
sodium chdroxide
Oc:ylamine plus sodium
hydroxide

Material Form*

Powder .
$1.0,1.5$, and $2.0 \%$

$0.51,0.99,1.96$, and $3.96 \%$

$1.10,2.22$, and $4.31 \%$

0.10 plus 1.00 and 1.00 plus $1.02 \%$

0.50 plus 1.0 and $0.56,1.07$, and $0.99 \%$

Type of Soil Treated Clay (Texas \#2)
Not given

Not given

Not given

Not given

Not given

Mixing

Capability

Good $\frac{\text { Type of Test }}{\text { Unconfined }}$

compression
Purpose of

$\frac{\text { Material }}{\text { Stabilizer }}$
Effective

Strength

$\frac{\text { Increase }}{\text { See comments }}$
Effectiveness

Excellent

Total Material Cost

Per $\mathrm{Cu} \mathrm{Ft}$

of Treated Soil

Not given
Test Agency

MIT
Test Report

Reference 39

Comments:

Samples treated with each additive at various percentages were-compared to samples treated only with cement. All samples were tested after cure of $1,4,7,28$, and 34 days followed by a 24-hour water immersion.

Effectiveness: The additives with rate of treatment (percent) are listed below in order of increase in strength over the cement only treated samples :

(Continued on next page)

* Basic material 
Ferric chloride ( 0.10 percent) plus sodium hydroxice ( 1.0 percent):

1 day cure - 209 percent strength increase

4 day cure - 236 percent strength increase

7 day cure - 136 percent strength increase

Octylamine ( 0.50 percent) plus sodium hydroxide ( 0.56 percent):

1 day cure - 144 percent strength increase

4 day cure - 131 percent strength increase

7 day cure -84 percent strength increase

Sodium aluminate ( 1.10 percent):

1 day cure - 142 percent strength increase

4 day cure - 123 percent strength increase

7 day cure -88 percent strength increase

Sodium hydroxide ( 1.0 percent):

1 day cure - 110 percent strength increase

4 day cure - 80 percent strength increase

7 day cure - 17 percent strength increase

The sodium sulfate was detrimental to the soil-cement mixture. 
Category*

Cement

Basic Material

Portland cement

Secondary Material

Sodium hydroxide

Sodium sulfate

Sodium aluminate

Sodium metasilicate

Material Form*

Powder

Type of Soil Treated

Clay (Texas \#2)

$0.97,1.99$, and $3.95 \%$

$1.13,2.26$, and $4.44 \%$

0.88 and $1.88 \%$

Mixing

Capability

Good

\begin{tabular}{llll} 
Type of Test & $\begin{array}{l}\text { Purpose of } \\
\text { Material }\end{array}$ & $\begin{array}{l}\text { Effective } \\
\text { Strength } \\
\text { Increase }\end{array}$ & Effectiveness \\
\cline { 5 - 6 } Compression & Stabilizer & See comments & Excellent
\end{tabular}

Total Material Cost

Per Cu Ft

$\frac{\text { of Treated Soil }}{\text { Not given }} \quad \frac{\text { Test Agency }}{\text { MIT }} \quad \frac{\text { Test Report }}{\text { Reference } 39}$

Comments:

Samples treated with each additive at various percentages were compared to samples with cement only.

Sodium hydroxide (2.35 percent) was effective in improving the strength of the soil with 10 percent cement. The increase in strength was 70 percent after one day cure and 91 percent after 34 days cure. Next in the order of improvement were sodium aluminate (2.26 percent and sodium metasilicate ( 1.88 percent which gave improvements of 41 percent (one day cure) and 74 percent ( 34 days cure), and 64 percent (one day cure) and 67 percent ( 34 days cure), respectively.

* Basic material 
Category*

Cement

Basic Material

Portland cement $\frac{\text { Rate of Material }}{5 \%}$

$1.0 \%$

$1.0 \%$

$1.0 \%$

Type of Soil Treated

Clay (Illinois)
Not given

Not given

Not given

Mixing

Capability

Good

Powder

Effective

Purpose of

Streng th

$\frac{\text { Type of Test }}{\text { Unconfined }}, \frac{\text { Material }}{\text { Stabilizer }}$

Increase

Effectiveness

See comments

Excellent

compression

Total Material Cost

Per Cu Ft

of Treated Soil

Not given

Test Agency

MIT
Test Report

Reference 38

\section{Comments:}

Cure was for 1, 4, and 7 days. Each sample was then subjected to 24 hours water immersion and tested. Samples treated with 5 percent cement and additive were compared to samples treated with 5 percent cement. Each secondary material was used with cement in treating samples.

Effectiveness: The sodium hydroxide ( 1 percent) was slightly effective. The increase in strength over the 5 percent only treated samples for 1,4 , and 7 days cure was 72,41 , and 36 percent. The other two additives were detrimental to the strength of the samples treated with the 5 percent cement.

(Continued on next page)

* Basic material 
NOTE: Further testing was conducted with sodium hydroxide $(0.5,1.0$, and 1.5 percent) as an additive for 5 and 10 percent cement for stabilizing. It was found that the optimum effectiveness for both 5 and 10 percent cement was sodium hydroxide at 1.0 percent. However, samples treated with 15 percent cement only had strengths of 143 percent and 13 percent greater than that for 5 and 10 percent cement plus sodium hydroxide, respectively. 
Category*

Cement

Basic Material

Rate of Material

Cost

Portland cement

$5 \%$

Not given

Secondary Material

Sodium hydroxide

Sodium sulfite

Sodium carbonate

Sodium metasilicate

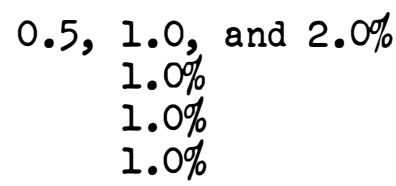

Not given

Not given

Not given

Not given

Mixing

Type of Soil Treated

Capability

Material Form*

Clay (Texas \#I)

Good

Powder

ose of

Type of Test

Purpose of
Material

Effective

Unconfined

Stabilizer

Strength

Increase

Effectiveness

compression

See comments

Excellent

Total Material Cost

Per Cu Ft

of Treated Soil

Test Agency

Test Report

Not given

MIT

Reference 38

Comments:

Samples treated with cement and each additive were compared to samples treated only with cement. Tests were run on samples after 1, 4, and 7 days cure followed by 24 hours water immersion.

Effectiveness: Samples treated with the additives sodium sulfite and sodium carbonate had lower strengths than samples treated with cement alone (detrimental).

(Continued on next page)

* Basic material 
The strength of cement with the additive sodium hydroxide was increased by 30 percent after one day cure and by 45 percent after seven days cure as compared to the same cure time for cement only treated samples. This material's effectiveness was slight.

Sodium metasilicate ( 1 percent) was next in effectiveness with somewhat lower values of strength increase. 
Category*

Cement

Basic Material

Portland cement

Secondary Material

Sodium hydroxide

Sodium hydorxide plus barium chloride

Sodium sulfite

Sodium carbonate

Sodium metasilicate

Material Form*

Powder
Rate of Material $5 \%$

$1 \%$

1.0 and $0.1 \%$

$1.0 \%$

$1.0 \%$

$1.0 \%$

Type of Soil Treated

Clay (Texas \#2)
Not given

Not given

Not given

Not given

Not given

Mixing

Capability

Good

$\begin{array}{llll}\text { Type of Test } & \begin{array}{l}\text { Purpose of } \\ \text { Material }\end{array} & \begin{array}{l}\text { Effective } \\ \text { Strength } \\ \text { Increase }\end{array} & \text { Effectiveness } \\ \begin{array}{c}\text { Unconfined } \\ \text { compression }\end{array} & \text { Stabilizer } & \text { See comments } & \text { Excellent }\end{array}$

Total Material Cost Per Cu Ft of Treated Soil

Not given

: Test Agency

MIT
Test Report

Reference 38

\section{Comments:}

The treated samples (with each additive) were compared to samples treated only with 5 percent cement. Cure time is listed below; however, before testing, the samples were also subjected to 24 hour water immersion.

( Continued, on next pase)

* Basic material 
Additive

None

Sodium hydroxide

Sodium hydroxide

plus barium chloride

Sodium sulfite

Sodium carbonate

Sodium metasilicate

$\begin{array}{crrr}\begin{array}{c}\% \\ \text { Additive }\end{array} & \begin{array}{c}\text { Curing } \\ \text { Days }\end{array} & \begin{array}{c}\text { Strength } \\ \text { psi }\end{array} \\ 0 & & 76 \\ & 4 & 103 \\ & 7 & 157\end{array}$

1.0

$\begin{array}{ll}1 & 162 \\ 4 & 185 \\ 7 & 184\end{array}$

1.0

0.1

1

4
7

115

195

232

1.0

$\begin{array}{rr}1 & 45 \\ 4 & 104 \\ 7 & 107\end{array}$

1.0

1
4
7

1.0
Strength Change

Based on Soil

Without

Additive 
Category*

Cement

Basic Material

Rate of Material

Cost

Portland cement

$5 \%$

Not given

Secondary Material

Sodium metasilicate

$1 \%$

Not given

Mixing

Material Form*

Type of Soil Treated

Capability

Powder

lean clay

Good

clay

Good

$\begin{array}{llll}\text { Type of Test } & \begin{array}{l}\text { Purpose of } \\ \text { Material }\end{array} & \begin{array}{l}\text { Effective } \\ \text { Strength } \\ \text { Increase }\end{array} & \text { Effectiveness } \\ \begin{array}{c}\text { Stabilizer } \\ \text { compression }\end{array} & \begin{array}{l}\text { Saterproofer } \\ \text { Semments }\end{array} & \text { See comments }\end{array}$

Total Material Cost

Per Cu Ft

$\frac{\text { of Treated Soil }}{\text { Not given }}$

Test Agency

WES

$\frac{\text { Test Report }}{\text { Reference } 25}$

Comments:

The untreated samples were unsuitable for compression tests after 4 days dry cure followed by 4 days wetting by capillary action.

Effectiveness: Lean clay - The treated samples possessed some compressive strength (115 psi); however, the samples were not waterproof.

Clay - The samples possessed no strength nor were they waterproof.

* Basic material 
Cátegory*

Cement

Basic Material

Portland cement
Rate of Material

$5 \%$
Cost

Not given

Secondary Material

Sodium orthosilicate

Material Form*

Powder
$1.0 \%$

Type of Soil Treated

Lean clay

Clay

Not given

Mixing

Capability

Good

Good

\begin{tabular}{|c|c|c|c|}
\hline Type of Test & $\begin{array}{c}\text { Purpose of } \\
\text { Material }\end{array}$ & $\begin{array}{l}\text { Effective } \\
\text { Strength } \\
\text { Increase } \\
\end{array}$ & Effectiveness \\
\hline $\begin{array}{l}\text { Unconfined } \\
\text { compression }\end{array}$ & $\begin{array}{l}\text { Stabilizer } \\
\text { Waterproofer }\end{array}$ & See comments & See comment \\
\hline
\end{tabular}

Total Material Cost

Per Cu Ft

$\frac{\text { of Treated Soil }}{\text { Not given }}$

$\frac{\text { Test Agency }}{\text { WES }}$

Test Report.

References 25

\section{Comments:}

The untreated samples were not suitable for compression tests after 4 days cure followed by 4 days wetting by capillary action.

Effectiveness: Lean clay -The samples possessed some compressive strength (83 psi); however, they were not waterproof.

Clay - The samples possessed no strength nor were they waterproof.

* Basic material 
Category*

Cement

Basic Material

Portland cement

$\frac{\text { Rate of Material }}{5 \%}$

Cost

Not given

Secondary Material

Sodium orthosilicate

Sodium metasilicate

Grade 50 silicate

Grade 40 silicate

Sodium oxide $\left(\mathrm{Na}_{2} \mathrm{O}\right)$

0.54 and $1.03 \%$

0.60 and $1.33 \%$

1.00 and $1.98 \%$

1.00 and $1.98 \%$

Not given

Not given

Not given

Not given

Silicon doixide $\left(\mathrm{SiO}_{2}\right)$

Material Form*

Type of Soil Treated

Powder

silt

Mixing

Capability

Good

\begin{tabular}{|c|c|c|c|}
\hline Type of Test & $\begin{array}{c}\text { Purpose of } \\
\text { Material }\end{array}$ & $\begin{array}{l}\text { Effective } \\
\text { Strength } \\
\text { Increase }\end{array}$ & Effectiveness \\
\hline $\begin{array}{l}\text { Unconfined } \\
\text { compression }\end{array}$ & $\overline{\text { Stabilizer }}$ & See comments & Excellent \\
\hline
\end{tabular}

Total Material Cost

Per Cu Ft

of Treated Soil

Not given

Comments:

Samples treated with each additive plus soda and silica at various percents were compared to samples treated with cement only. All seinples were tested after the cure time shown below followed by a 24-hour water immersion. The additive (percent) which gave the best results is given below.

(Continued on next page)

* Basic material 


\begin{tabular}{|c|c|c|c|c|c|}
\hline Additive & $\begin{array}{c}\text { Additive } \\
\% \\
\end{array}$ & $\begin{array}{r}\text { Ratio } \\
\mathrm{Na}_{2} \mathrm{O} \\
\text { to } \\
\mathrm{SiO}_{2} \\
\end{array}$ & $\begin{array}{c}\text { Curing } \\
\text { Days } \\
\end{array}$ & $\begin{array}{l}\text { Strength } \\
\text { psi }\end{array}$ & $\begin{array}{c}\text { Based on } \\
\text { Soil Cement } \\
\text { Without } \\
\text { Additive } \\
\%\end{array}$ \\
\hline None & 0 & 0 & $\begin{array}{r}1 \\
4 \\
7 \\
28\end{array}$ & $\begin{array}{r}80 \\
90 \\
95 \\
125\end{array}$ & $\begin{array}{l}-- \\
-- \\
-- \\
--\end{array}$ \\
\hline $\begin{array}{l}\text { Sodium } \\
\text { orthosilicate }\end{array}$ & 1.03 & $2: 1$ & $\begin{array}{r}1 \\
4 \\
7 \\
28\end{array}$ & $\begin{array}{l}217 \\
235 \\
286 \\
491\end{array}$ & $\begin{array}{l}+171 \\
+161 \\
+201 \\
+293\end{array}$ \\
\hline $\begin{array}{l}\text { Sodium } \\
\text { metasilicate }\end{array}$ & 1.33 & $1: 1$ & $\begin{array}{r}1 \\
4 \\
7 \\
28\end{array}$ & $\begin{array}{l}135 \\
198 \\
218 \\
344\end{array}$ & $\begin{array}{r}+69 \\
+120 \\
+129 \\
+175\end{array}$ \\
\hline $\begin{array}{l}\text { Grade } 50 \\
\text { silicate }\end{array}$ & 1.00 & $1: 2$ & $\begin{array}{r}1 \\
4 \\
7 \\
28\end{array}$ & $\begin{array}{l}123 \\
370 \\
420 \\
553\end{array}$ & $\begin{array}{r}+54 \\
+311 \\
+342 \\
+342\end{array}$ \\
\hline $\begin{array}{l}\text { Grade } 40 \\
\text { silicate }\end{array}$ & 1.00 & $1: 3.22$ & $\begin{array}{r}1 \\
4 \\
7 \\
28\end{array}$ & $\begin{array}{l}290 \\
352 \\
386 \\
530\end{array}$ & $\begin{array}{l}+263 \\
+291 \\
+306 \\
+324\end{array}$ \\
\hline
\end{tabular}

Effectiveness: All additives shown above were very effective in increasing the strength of the soil-cement samples.

Grade 40 silicate-treated samples developed the highest initial (one-day) strength.

Grade 50 developed the highest (28 days) strength followed closely by Grade 40 silicate. 
Category*

Cement

Basic Material

Portland cement
Rate of Material

$5,6,8$, and $10 \%$
. Cost

$1.5 \phi$ per $1 \mathrm{~b}$

Secondary Material

See comments

Sodium sulfate

$1 \%$

$10 \phi$ per $1 \mathrm{~b}$

Mixing

Material Form*

Type of Soil Treated

Powder

Loess

Capability

Good

Effectiveness

Type of Test

Purpose of

Effective

Material

Streng th

Stabilizer

Increase

See comments

Excellent

Total Material Cost

Per Cu Ft

of Treated Soil

Test Agency

Test Report

$\$ 1.60$ (exclusive of shipping, WES

Reference 14

storing, and construction)

Comments:

Samples were molded in a Harvard Miniature Compaction Apparatus in five layers (each layer compacted with ten tamps of a 40-lb spring tamper). Samples were cured at 100 percent relative humdity for 24 hours and subjected to tests. When the strength of the treated samples as compared to untreated samples (25 psi) increased from 25 psi to 100 psi or greater, the materials were considered to warrant further consideration as stabilizers.

(Continued on next page)

* Basic material 


\begin{tabular}{|c|c|c|c|c|}
\hline Additive & $\begin{array}{c}\text { Additive } \\
\% \\
\end{array}$ & $\begin{array}{l}\text { Unconfined } \\
\text { Compression } \\
\text { Strength } \\
\text { psi } \\
\end{array}$ & $\begin{array}{l}\text { Strength } \\
\text { Increase } \\
\text { as Compared } \\
\text { to Untreated } \\
\text { Soil } \\
\end{array}$ & $\begin{array}{l}\text { Strength } \\
\text { Increase } \\
\text { Compared } \\
\text { to } \\
\text { Cement Without } \\
\text { Additive } \\
\end{array}$ \\
\hline None & 0 & 24 & - & - \\
\hline $\begin{array}{l}\text { Portland cement with: } \\
\text { Sodium carbonate } \\
\text { Sodium hydroxide } \\
\text { Sodium sulfate } \\
\text { Sodium sulfite } \\
\text { Potassium } \\
\text { permanganate }\end{array}$ & $\begin{array}{l}5 \\
1 \\
1 \\
1 \\
1\end{array}$ & $\begin{array}{r}160 \\
167 \\
90 \\
207 \\
127\end{array}$ & $\begin{array}{l}+567 \\
+596 \\
+275 \\
+763 \\
+429\end{array}$ & $\begin{array}{c}+4^{-} \\
\text {Negative } \\
+29 \\
\text { Negative }\end{array}$ \\
\hline Portland cement & 6 & 165 & +588 & +3 \\
\hline Portland cement & 8 & 175 & +629 & +9 \\
\hline Portland cement & 10 & 209 & +771 & +31 \\
\hline \multicolumn{5}{|c|}{$\begin{array}{l}\text { Portland cement ( } 5 \text { percent ) with } 1 \text { percent sodium sulfate gave the bes } \\
\text { results. Portland cement ( } 10 \text { percent) gave a slight increase over the } \\
\text { combination of the two materials. } \\
\text { Traffic tests were conducted on a lean clay soil treated with } 5 \text { per- } \\
\text { cent portland cement and } 1 \text { percent sodium sulfate and the strength } \\
\text { developed was sufficient to meet the requirements of emergency } \\
\text { military roads. }\end{array}$} \\
\hline
\end{tabular}


Category*

Cement

Basic Material

Portland cement

Secondary Material

Sodium sulfate

ET-224 dispersant

Barium chloride

Sodium fluosilicate
Rate of Material

$5 \%$

$0.5 \%$

$0.1 \%$

$1.0 \%$

$1.0 \%$

Not given

Not given

Not given

Not given

Mixing

Type of Soil Treated

Loess

Capability

Good

Powder

Purpose of

Effective

Strength

Type of Test

Material

Increase

Unconfined

compression

see comments

Effectiveness

Excellent

Total Material Cost

Per $\mathrm{Cu} F t$

of Trea
Not given
Comments:

The treated samples with the additive were compared to samples treated with 5 percent cement. Curing time is listed below: however, before testing the samples were also subjected to 24 hours water immersion.

(Continued on next page)

* Basic material 
Additive

None

Sodium sulfate

ET-224 Dispersant

Barium chloride

Sodium fluosilicate

\begin{tabular}{|c|c|c|}
\hline $\begin{array}{c}\% \\
\text { Additive }\end{array}$ & $\begin{array}{c}\text { Curing } \\
\text { Days } \\
\end{array}$ & $\begin{array}{c}\text { Strength } \\
\text { psi }\end{array}$ \\
\hline 0 & $\begin{array}{l}1 \\
4 \\
7\end{array}$ & $\begin{array}{l}145 \\
172 \\
195\end{array}$ \\
\hline
\end{tabular}

0.5

1
4
7

0.1

$\begin{array}{ll}1 & 165 \\ 4 & 260 \\ 7 & 304\end{array}$

217

247

275

304

1.0

$\begin{array}{ll}1 & 100 \\ 4 & 145 \\ 7 & 172\end{array}$

1.0
Strength Change

Based on Soil Without Additive $\%$

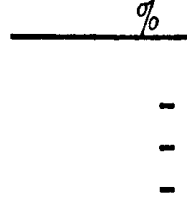

$$
\begin{array}{r}
+50 \\
+44 \\
+41 \\
+14 \\
+51 \\
+56
\end{array}
$$

Negative Negative Negative -

Negative Negative Negative

Effectiveness: Sodium sulfate ( 0.5 percent) and ET-22 4 dispersant ( 0.1 percent) were effective in combination with 5 percent cement for stabilizing loess soil. 


\section{Category*}

Cement

Basic Material

Rate of Material

Cost

Portland cement

$5 \%$

Not given

Secondary Material

Sodium sulfate

Sodium metasilicate

Material Form*

Powder
$1 \%$
$1 \%$

Type of Soil Treated

Lean clay

Clay

Effective

Purpose of Strength

Type of Test

Unconfined compression
Material

Stabilizer Waterproofer
Not given

Not given

Mixing

Capability

Good

Good

Total Material Cost

Per Cu Ft

of Treated Soil

Not given

Test Agency

WES
Test Report

Reference 25

Comments:

The untreated samples were not suitable for compression tests after 4 days cure followed by 4 days wetting by capillary action.

Effectiveness: Lean clay - The treated samples possessed some compressive strength (96 psi)! however, the samples were not waterproof. proof.

Clay - The samples possessed no strength nor were they water-

* Basic material 
Category*

Cement

Basic Material

Portland cement
Rate of Material $10 \%$
Cost

Not given

Secondary Material

Sulfate compounds

(see comments)

Material Form*

Powder
Type of Soil Treated

Sand (Wisconsin \#1)
Mixing

Capability

Good

$\begin{array}{llll}\text { Type of Test } & \begin{array}{c}\text { Purpose of } \\ \text { Material }\end{array} & \frac{\begin{array}{l}\text { Effective } \\ \text { Strength } \\ \text { Increase }\end{array}}{\text { Stabilizer }} & \frac{\text { Effectiveness }}{\text { Sempression }}\end{array}$

Total Material Cost

Per Cu Ft

$\frac{\text { of Treated Soil }}{\text { Not given }} \quad \frac{\text { Test Agency }}{\text { MIT }} \frac{\text { Test Report }}{\text { Reference } 39}$

\section{Comments:}

Samples treated with each sulfate plus cement were compared to samples treated with cement only. Cure time is shown below; however, in addition to this time, samples prior to testing were-immersed in water 24 hours. Each additive was tested at several rates; however, the most effective is shown. 'Also, methods of adding additive were solution, slurry, and dry mix with cement. The most effective method is given.

(Continued on next page)

* Basic material 


\begin{tabular}{|c|c|c|c|c|c|}
\hline Additive & $\begin{array}{l}\text { Additive } \\
\% \\
\end{array}$ & $\begin{array}{l}\text { Method } \\
\text { of } \\
\text { Adding } \\
\text { Additives } \\
\end{array}$ & $\begin{array}{c}\text { Curing } \\
\text { Days } \\
\end{array}$ & $\begin{array}{l}\text { Strength } \\
\text { psi }\end{array}$ & $\begin{array}{c}\text { Strength } \\
\text { Change } \\
\text { Based on } \\
\text { Soil Cement } \\
\text { Without } \\
\text { Additive } \\
\% \\
\end{array}$ \\
\hline None & 0 & -- & $\begin{array}{r}1 \\
4 \\
7 \\
28\end{array}$ & $\begin{array}{l}25 \\
20 \\
19 \\
23\end{array}$ & $\begin{array}{l}-- \\
-- \\
--\end{array}$ \\
\hline Sodium sulfate & 1.08 & Solution & $\begin{array}{r}1 \\
4 \\
7 \\
28\end{array}$ & $\begin{array}{l}205 \\
350 \\
342 \\
425\end{array}$ & $\begin{array}{r}+720 \\
+1650 \\
+1700 \\
+1748\end{array}$ \\
\hline $\begin{array}{l}\text { Calcium sulfate } \\
\text { anhydrite }\end{array}$ & 1.10 & Slurry & $\begin{array}{r}1 \\
4 \\
7 \\
28\end{array}$ & $\begin{array}{l}165 \\
280 \\
363 \\
413\end{array}$ & $\begin{array}{r}+560 \\
+1300 \\
+1810 \\
+1696\end{array}$ \\
\hline $\begin{array}{l}\text { Calcium sulfate } \\
\text { hydrate (gypsum) }\end{array}$ & 1.10 & slurry & $\begin{array}{r}1 \\
4 \\
7 \\
28\end{array}$ & $\begin{array}{l}183 \\
271 \\
292 \\
378\end{array}$ & $\begin{array}{r}+632 \\
+1255 \\
+1437 \\
+1543\end{array}$ \\
\hline Magnesium sulfate & 0.48 & Solution & $\begin{array}{r}1 \\
4 \\
7 \\
28\end{array}$ & $\begin{array}{l}167 \\
193 \\
227 \\
304\end{array}$ & $\begin{array}{r}+568 \\
+865 \\
+1095 \\
+1222\end{array}$ \\
\hline
\end{tabular}

Effectiveness: The additives above are listed in the order of their effectiveness. However, all additives were very effective in increasing the strength of the cement-treated samples. The lowest increase in effectiveness was 308 percent. 
Category*

Cement

Basic Material

Rate of Material

Cost

Type I normal

$5 \%$

Not given

portland cement

Secondary Material

Chemical additives

(see comments)

0.5 and $1 \%$

Not given

Material Form*

Type of Soil Treated

Mixing

Capability

Powder

Loess

Good

\begin{tabular}{|c|c|c|c|}
\hline Type of Test & $\begin{array}{c}\text { Purpose of } \\
\text { Material }\end{array}$ & $\begin{array}{l}\text { Effective } \\
\text { Strength } \\
\text { Increase } \\
\end{array}$ & Effectiveness \\
\hline $\begin{array}{l}\text { Unconfined } \\
\text { compression }\end{array}$ & Stabilizer & See comments & Excellent \\
\hline
\end{tabular}

Total Material Cost

Per Cu Ft

of Treated Soil

Test Agency

Test Report.

Not given

MIT

Reference 36

Comments:

The treated samples with additives were compared to soil-cement treated samples. Samples were cured for 7 and 28 days at room temperature in 100 percent relative humidity and then immersed in water for 24 hours. The soil-cement strength after a 7-day cure without additive was $180 \mathrm{psi}$ and 260 psi after a 28-day cure.

(Continued on next page)

* Basic material 
Strength Change Based on

Additive

Quadrafos

Lignosol X2D

Polyvinyl alcohol (50-42)

Piccolyte S125

Picco XX-100B

Vinsol

Arquad $2 \mathrm{HT}$

Calcium hydroxide

Sodium hydroxide

Sodium sulfite

Sodium carbonate
Percent 7-Day Cure

0.5

$+22$

0.5

1.0

1.0

0.5

0.5

1.0

0.5

0.5

0.5

0.5

1.0

0.5

1.0

0.5

1.0
$+22$

$+47$

$+25$

$+3$

$+25$

$+28$

$+8$

$+6$

$+14$

$+89$

$+87$

$+81$

$+67$

$+44$

$+72$
Negative

Negative $+6$

Negative

Negative

0

Negative

Negative

Negative

Negative

$+49$

$+77$

$+15$

$+32$

$+11$

$+27$

Other chemical additives used with 5 percent cement-treated soil. samples were as follows:

Pozzolith 2AA

Daxad 21

Arcolor 4465

Phosphorus pentoxide

Darex polyvinyl acetate X52L
Ferric sulphate

Ferric chloride

Calcium chloride

Sodium chloride

Potassium permanganate

These materials, when used, either gave no increase in compressive strength over the 5 percent cement treated samples or gave less strength (chemicals were detrimental to strength).

(Continued on next page) 
Effectiveness. As seen from the percent increase in compressive strength when the additives were used, only sodium hydroxide ( 1 percent rate) gave any significant increase in strength. Sodium sulfite and sodium carbonate gave the nex $t$ highest increase in strength.

Samples with 10 percent of cement without additives have strength of 415 and 525 psi for 7 and 28 days cure, respectively. These values are 135 percent over the strength value for the 5 percent of cement (plus additives), 7-day cure, and 102 percent over the strength value for the 5 percent of cement (plus additives), 28-day cure. 


\begin{tabular}{|c|c|c|}
\hline $\begin{array}{l}\text { Category* } \\
\text { Cement }\end{array}$ & & \\
\hline Basic Material & Rate of Material & Cost \\
\hline $\begin{array}{l}\text { Type I normal } \\
\text { portland cement }\end{array}$ & $5 \%$ & Not given \\
\hline Secondary Mater & ial & \\
\hline $\begin{array}{l}\text { Chemical additives } \\
\text { (see comments) }\end{array}$ & 0.5 and $1.0 \%$ & Not given \\
\hline Material Form* & Type of Soil Treated & $\begin{array}{c}\text { Mixing } \\
\text { Capability } \\
\end{array}$ \\
\hline Powder & Silt & Good \\
\hline Týpe of Test & $\begin{array}{l}\text { Effective } \\
\text { Strength } \\
\text { Increase } \\
\end{array}$ & Effectiveness \\
\hline $\begin{array}{l}\text { Unconfined } \\
\text { compression }\end{array}$ & See comments & Excellent \\
\hline
\end{tabular}

Total Material Cost Per Cu Ft

$\frac{\text { of Treated Soil }}{\text { Not given }} \cdot \frac{\text { Test Agency }}{\text { MIT }} \frac{\text { Test Report }}{\text { Reference } 36}$

\section{Comments:}

The treated samples with additives were compared to soil-cement treated samples. Samples were cured for 7 and 28 days at room temperature in 100 percent relative humidity and then imersed in water for 24 hours.

(Continued on next page)

* Basic material 


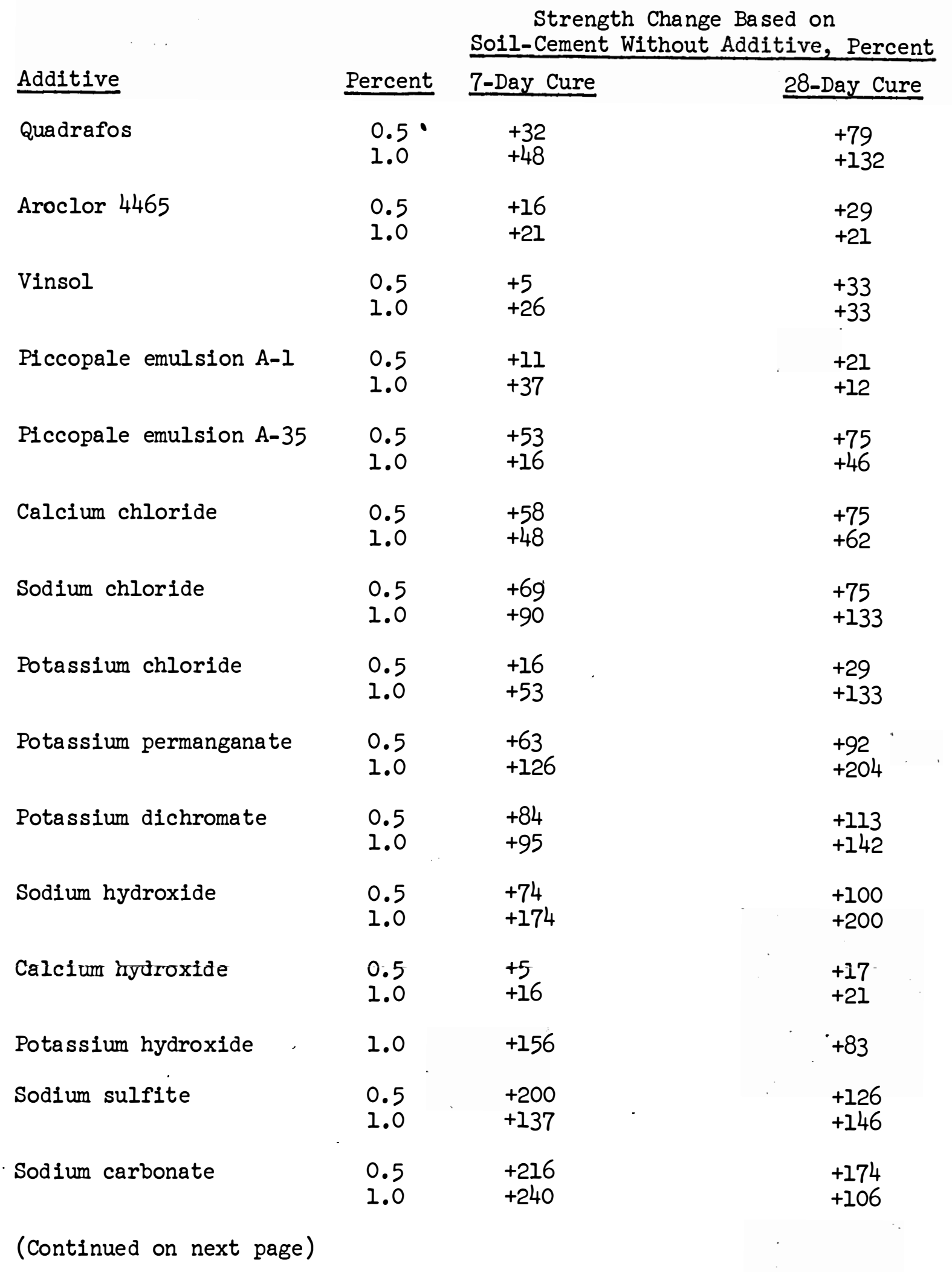


Other chemical additives used with 5 percent cement-treated soil samples were as follows:

Pozzolith 2AA

Daxad 21

Lignosol X2D

Losorb

PVA $(5-88)$

PVA $(5-88)+$ Paraformaldehyde
PVA $(65-98)$

PVA (65-98) + paraformaldehyde

Phosphorus pentoxide

Borax

These materials when used either gave no increase in compressive strength over the 5 percent cement treated samples or gave less strength (chemicals were detrimental to the strength).

Effectiveness. As seen from the percent increase in compressive strength when the additives were used, sodium hydroxide ( 1 percent rate), potassium permanganate ( 1 percent rate), sodium carbonate ( 0.5 and 1.0 percent rates), and sodium sulfite ( 0.5 and 1.0 percent rates) were quite effective. Potassium hydroxide ( 1.0 percent rate), potassium dichromate ( 0.5 and 1.0 percent rates), sodium chloride ( 1.0 percent rate), and potassium chloride ( 1.0 percent rate) were next in order of effectiveness. 
Category*

Cement

Basic Material

Type I normal

portland cement

Secondary Material

Chemical additives

(see comments)

Material Form*

Powder
Rate of Material $5 \%$

0.5 and $1 \%$

Type of Soil Treated

Silty clay
Cost

Not given
Not given

Mixing Capability

Good

\begin{tabular}{|c|c|c|c|}
\hline Type of Test & $\begin{array}{c}\text { Purpose of } \\
\text { Material }\end{array}$ & $\begin{array}{l}\text { Effective } \\
\text { Strength } \\
\text { Increase }\end{array}$ & Effectiveness \\
\hline $\begin{array}{l}\text { Unconfined } \\
\text { compression }\end{array}$ & Stabilizer & See comments & Excellent \\
\hline
\end{tabular}

Total Material Cost

Per $\mathrm{Cu} \mathrm{Ft}$

of Treated Soil

Not given

Test Agency

MIT

Test Report

Reference 36

\section{Comments:}

The treated samples with additives were compared to soil-cement treated samples. Samples were cured (for 7 and 28 days) at room temperature in 100 percent relative humidity and then immersed in water for 24 hours. The soil-cement strength after a 7-day cure without additive was $300 \mathrm{psi}$ and 435 psi after a 28-day cure.

(Continued on next page)

* Basic material 
Strength Change Based on Soil-Cement Without Additive

\begin{tabular}{|c|c|c|c|}
\hline \multirow{2}{*}{ Additive } & \multirow[b]{2}{*}{ Percent } & \multicolumn{2}{|c|}{$\%$} \\
\hline & & 7-Day Cure & 28-Day Cure \\
\hline Aroclor 4465 & $\begin{array}{l}0.5 \\
1.0\end{array}$ & $\begin{array}{l}+12 \\
+31\end{array}$ & $\begin{array}{l}+23 \\
+23\end{array}$ \\
\hline Vinsol & $\begin{array}{l}0.5 \\
1.0\end{array}$ & $\begin{array}{l}+12 \\
+20\end{array}$ & $\begin{array}{c}\text { Negative } \\
+3\end{array}$ \\
\hline Sodium chloride & $\begin{array}{l}0.5 \\
1.0\end{array}$ & $\begin{array}{l}+7 \\
+6\end{array}$ & $\begin{array}{l}\text { Negative } \\
+10\end{array}$ \\
\hline Potassium chloride & 0.5 & +6 & Negative \\
\hline Potassium permanganate & 1.0 & +65 & +43 \\
\hline Darex polyvinyl & 0.5 & +6 & Negative \\
\hline Quadrafos & $\begin{array}{l}0.5 \\
1.0\end{array}$ & $\begin{array}{l}+38 \\
+105\end{array}$ & $\begin{array}{l}+38 \\
+105\end{array}$ \\
\hline Sodium hydroxide & $\begin{array}{l}0.5 \\
1.0\end{array}$ & $\begin{array}{l}+169 \\
+174\end{array}$ & $\begin{array}{l}+265 \\
+215\end{array}$ \\
\hline Sodium sulfite & $\begin{array}{l}0.5 \\
1.0\end{array}$ & $\begin{array}{l}+7 \\
+130\end{array}$ & $\begin{array}{l}+33 \\
+174\end{array}$ \\
\hline Sodium carbonate & $\begin{array}{l}0.5 \\
1.0\end{array}$ & $\begin{array}{l}+93 \\
+200\end{array}$ & $\begin{array}{l}+112 \\
+199\end{array}$ \\
\hline
\end{tabular}

Other chemical additives used with 5 percent cement soil-treated samples were as follows:

$\begin{array}{ll}\text { Polyvinyl alcohol }(50-42) & \begin{array}{l}\text { Arquad } 2 \mathrm{HT} \\ \text { Acetate X52L } \\ \text { Piccolyte Sl25 }\end{array} \\ \text { Potassium hydroxide } & \text { Calcium chloride } \\ \text { Ferric chloride } & \text { PVA }(5-88) \\ \text { Ferric sulfate } & \text { PVA }(5-88)+\text { paraformaldehyde }\end{array}$

Phosphorus pentoxide

These materials, when used, either gave no increase in compressive strength over the 5 percent cement-treated samples or gave less strength (chemicals were detrimental to strength).

(Continued on next page) 
Effectiveness. As seen from the percent increase in compressive strength when the additives were used, only potassium permanganate (1 percent rate), Quadrafos (1 percent rate), sodium hydroxide (0.5 and 1 percent rates), sodium sulfite (1 percent rate), and sodium carbonate ( 0.5 and 1 percent rates) showed any real effectiveness.

Samples with 10 percent of cement without additives had strength of 560 and 665 psi for 7- and 28-days curing, respectively. These value $s$ are 87 percent over the strength value for 5 percent of cement (7-day cure) and 53 percent over the strength value for 5 percent of cement and 28-day cure.

The chemical additive $s$ [Quadrafos ( 1 percent rate), sodium hydroxide ( 0.5 and 1 percent rates), sodium sulfite ( 1 percent rate), and sodium carbonate ( 0.5 and 1 percent rate $s)$ ] are the only one $s$ that, when used with 5 percent of cement-treated samples, exceeded the strength of samples treated only with 10 percent of cement. 
Category*

Cement

Basic Material

Type I portland cement
Rate of Material

$6 \%$ (on lean clay)

$5 \%$ (on heavy clay)

Secondary Material Sodium hyd roxide (with heavy clay only)

Powder
Material Form*

Type of Soil Treated

Lean clay Heavy clay

Effective

Purpose of Strength

$\frac{\text { Type of Test }}{\text { Unconfined }}$ compression

and traffic
Increase

See comments
Cost

Not given

Not given

Mixing

Capability

Good

Good

Total Material Cost Per Cu Ft

$\frac{\text { of } \operatorname{Tre}}{\text { Not given }}$

Test Agency

WES
Test Report

Reference 9

\section{Comments:}

Treated samples were compared to untreated samples (18 psi). Samples were prepared using the Harvard Miniature Compaction Apparatus in five layers (each layer was compacted with ten tamps of a 40-1b spring tamper). Samples were tested after 24 hours cure under 100 percent relative humidity and after 24 hours cure under 100 percent relative humidity followed by immersion in water for 24 hours.

(Continued on next page)

* Basic material 
Laboratory tests: The 6 percent portland cement treated lean clay in unconfined compression tests met the requirements of Category 2 stabilization, and 5 percent portland cement with 1 percent sodium hydroxide with heavy clay soil also met the Category 2 requirements.

Traffic tests: The materials as listed for the laboratory tests also met the requirements for emergency military operations. 


\section{Category: Lime}


Category*

Lime

Basic Material

Hydrated lime
Rate of Material

$2.5,4$, and $5 \%$
Cost

Not given

Secondary Material

Additives: Sodium hydroxide $1 \%$

Sodium sulfate, sodium carbonate, $1 \%$

Magnesium sulfate, calcium oxide 1 and $2 \%$

Calcium hydroxide

Portland cement

Material Form*

Powder

Purpose of

Type of Test

Unconfined compression
$2.5 \%$
Type of Soil Treated

Lean clay
Not given

Not given

Not given

Not given

Not given

Mixing

Capability

Good

Total Material Cost

Per Cu Ft

of Treated Soil

Not given
Test Agency

WES
Effective

Strength

$\frac{\text { Increase }}{\text { See comments }} \frac{\text { Effectiveness }}{\text { Excellent }}$ 
Ca tegory*

Lime

Basic Material

Rate of Material

Cost

Calcium hydroxide

$6.6 \%$

Not given

(slaked lime)

Secondary Material

Material Form*

Solid (lumps)

Type of Soil Treated

Clay (Vicksburg)

Effective

Strength

Type of Test

Unconfined

compression

Total Material Cost

Per $\mathrm{Cu} \mathrm{Ft}$

of Treated Soil

Not given

Purpose of

Increase

See comments

Effectiveness

Excellent
Test Report

Reference 41
Mixing

Capability

Good

Comments:

Treated samples were not compared to untreated samples. Tests were conducted after a 24 hour humid cure followed by a 24 hour wate $r$ immersion.

The strength of the treated samples was 150 psi.

* Basic material 
Category*

Lime

Basic Material

Rate of Material

Cost

Calcium hydroxide

$6.6 \%$

Not given

(slaked lime)

Secondary Material

Magnesium sulfate

$1.25 \%$

Not given

Material Form*

Type of Soil Treated

Mixing

Powder

Clay (Vicksburg)

Capability

Good

\begin{tabular}{|c|c|c|c|}
\hline Type of Test & $\begin{array}{l}\text { Purpose of } \\
\text { Material }\end{array}$ & $\begin{array}{l}\text { Effective } \\
\text { Strength } \\
\text { Increase }\end{array}$ & Effectiveness \\
\hline $\begin{array}{l}\text { Unconfined } \\
\text { compression }\end{array}$ & $\overline{\text { Stabilizer }}$ & See comments & Excellent \\
\hline
\end{tabular}

Total Material Cost

Per Cu Ft

of Treated Soil

Not given

Test Agency

Test Report

Reference 41

\section{Comments:}

Treated samples with additive compared to samples treated only with basic material. Tests conducted after a 24 hour humid cure followed by a 24 hour water immer sion.

The strength of the samples was 165 psi which was an increase of 10 percent over those with only the hydroxide (150 psi).

* Basic material 
Category*

Lime

Basic Material

Calcium and magne sium

limes ( $\mathrm{DaO}$ and $\mathrm{MgO}$ )
Rate of Material $\mathrm{CaO}-3,2$, and $1 \%$ $\mathrm{MgO}-1,2$, and 3\%
Cost

Not given

Secondary Material

Magne sium sulfate

$1 \%$

Not given

Mixing

Material Form*

Type of Soil Treated

Powder

Lean clay

Capability

Good

$\frac{\text { Type of Test }}{\begin{array}{c}\text { Unconfined } \\ \text { compression }\end{array}} \quad \frac{\begin{array}{c}\text { Purpose of } \\ \text { Material }\end{array}}{\text { Stabilizer }} \quad \frac{\begin{array}{c}\text { Effective } \\ \text { Strength } \\ \text { Increase }\end{array}}{\text { See comments }} \quad \frac{\text { Effectiveness }}{\text { Excellent }}$

Total Material Cost

Per Cu Ft

of Treated Soil

Not given

Test Agency

WES
Test Report

Internal Data

(1961), not

published

Comments:

Treated samples were compared to untreated samples (20 psi) and to samples treated with 4 percent calcium oxide plus-1-percent magnesium sulfate (139 psi). The samples were cured at 100 percent relative humidity for one day and then tested for Category 2 stabilization.

(Continued on next page)

* Basic material 
Effectiveness: The only combination of materials on lean clay which gave an increase over the 4 percent $\mathrm{CaO}$ plus 1 percent $\mathrm{MgSO}_{4}$ was 3 percent $\mathrm{CaO}$ plus 1 percent $\mathrm{MgSO}_{4}$ plus 1 per cent $\mathrm{MgO}(154 \mathrm{psi})$.

On the heavy clay soil, 3 percent $\mathrm{CaO}$ plus 1 percent $\mathrm{MgO}$ plus 1 percent $\mathrm{MgSO}_{4}$ was effective (162 psi). 
Category*

Lime

Basic Material

Rate of Material

Cost

Calcium oxide

$1,2,5$, and $7 \%$

Not given

Secondary Material

Material Form*

Solid (lumps)
Type of Soil Treated

Clay (Houston black)
Mixing

Capability

Good

$\frac{\text { Type of Test }}{\begin{array}{c}\text { Unconfined } \\ \text { compression }\end{array}} \quad \frac{\begin{array}{c}\text { Purpose of } \\ \text { Material }\end{array}}{\text { Stabilizer }} \quad \frac{\begin{array}{l}\text { Effective } \\ \text { Strength } \\ \text { Increase }\end{array}}{\text { See comments }} \quad \frac{\text { Effectiveness }}{\text { Excellent }}$

Total Material Cost

Per Cu Ft

of Treated Soil

Not given

Test Agency

MIT
Test Report

Reference 41

\section{Comments:}

Treated samples not compared to untreated samples. Tests were conducted ater a 24 hour humid cure followed by a 24 hour water immersion.

Effectiveness: Two percent calcium oxide added to the soil gave the highest strength ( 315 psi). The next highest strength was 260 psi at the 5 percent rate.

* Basic material 
Category*

Lime

Basic Material

Rate of Material

Cost

Calcium oxide (lime)

$5 \%$

Not given

Secondary Material

Material Form*

Solid (lumps)
Type of Soil Treated

Clay (Vicksburg)
Mixing

Capability

Good

\begin{tabular}{|c|c|c|c|}
\hline Type of Test & $\begin{array}{c}\text { Purpose of } \\
\text { Material }\end{array}$ & $\begin{array}{l}\text { Effective } \\
\text { Strength } \\
\text { Increase } \\
\end{array}$ & Effectiveness \\
\hline $\begin{array}{l}\text { Unconfined } \\
\text { compression }\end{array}$ & Stabilizer & See comments & Excellent \\
\hline
\end{tabular}

Total Material Cost

Per $\mathrm{Cu} \mathrm{Ft}$

of Treated Soil

Not given

Test Agency

Test Report

MIT

Reference 41

Comments:

Treated samples were not compared to untreated samples. Tests conducted after a 24 hour humid cure followed by a 24 hour water immersion.

Strength of the treated samples was 125 psi.

* Basic material 
Category*

Lime

Basic Material

Rate of Material

Cost

Calcium oxide

$5 \%$

Not given

Secondary Material

Additives (see comments)

Material Form*

Type of Soil Treated

Mixing

Capability

Solid (lumps)

Clay (Houston black)

Good

\begin{tabular}{|c|c|c|c|}
\hline Type of Test & $\begin{array}{c}\text { Purpose of } \\
\text { Material }\end{array}$ & $\begin{array}{l}\text { Effective } \\
\text { Strength } \\
\text { Increase } \\
\end{array}$ & Effectiveness \\
\hline $\begin{array}{l}\text { Unconfined } \\
\text { compression }\end{array}$ & Stabilizer & See comments & Excellent \\
\hline
\end{tabular}

Total Material Cost

Per Cu Ft

of Treated Soil

Not given

Test Agency

MIT
Test Report

Reference 41

Comments:

Samples treated with additives compared to samples treated with 5 percent calcium oxide ( $260 \mathrm{psi}$ strength). Tests conducted ater a 24 hour humid cure followed by a 24 hour water immersion.-

(Continued on next page)

* Basic material 
Strength Change Based on Samples Treated

\begin{tabular}{|c|c|c|c|}
\hline Additive & $\begin{array}{l}\text { Additive } \\
\% \\
\end{array}$ & $\begin{array}{l}\text { Strength } \\
\text { psi }\end{array}$ & $\begin{array}{c}\text { with Calcium Oxide } \\
\%\end{array}$ \\
\hline None & 0 & 260 & -- \\
\hline Magnesium sulfate & 1.25 & 390 & +50 \\
\hline Sodium metasilicate & 1.57 & 345 & +33 \\
\hline $\begin{array}{l}\text { Magnesium sulfate plus } \\
\text { sodium metasilicate }\end{array}$ & $\begin{array}{l}1.25 \\
1.37\end{array}$ & 505 & +94 \\
\hline Zinc sulfate & 1.46 & 205 & Negative \\
\hline Nickel sulfate & 1.34 & 450 & +73 \\
\hline
\end{tabular}

Effectiveness: All additives except zinc sulfate gave higher strength than samples with the calcium oxide only.

Magnesium sulfate ( 1.25 percent) plus sodium metasilicate ( 1.57 percent) were additives which gave the most improvement in strength. 
Category*

\section{Lime}

Basic Material

Calcium oxide
Rate of Material

$5 \%$
Cost

Not given

Secondary Material

See comments for additives

Material Form*

Solid (lumps)
Type of Soil Treated

Clay (Vicksburg)

Effective

Purpose of Strength

Type of Test

Unconfined

compression
Material

Stabilizer
Mixing

Capability

Good

Total Material Cost

Per Cu Ft

of Treated Soil

Not given

Test Agency

MIT
Test Report

Reference 41

\section{Comments:}

Samples treated with additives compared to samples treated with. calcium oxide only. Tests we re conducted ater a 24 hour humid cure followed by a 24 hour water immersion.

(Continued on next page)

* Basic material 


\begin{tabular}{|c|c|c|c|}
\hline Additive & $\begin{array}{c}\text { Additive } \\
\% \\
\end{array}$ & $\begin{array}{l}\text { Strength } \\
\text { psi }\end{array}$ & $\begin{array}{c}\text { Without Additive } \\
\%\end{array}$ \\
\hline None & 0 & 125 & -- \\
\hline Magnesium carbonate & 0.47 & 115 & Negative \\
\hline Magnesium fluoride & 0.32 & 125 & 0 \\
\hline Magnesium oxide & 0.20 & 110 & Negative \\
\hline Ammonium chloride & 2.50 & 140 & +12 \\
\hline Sodium metasilicate & 1.30 & 170 & +36 \\
\hline $\begin{array}{l}\text { Sodium metasilicate plus } \\
\text { magnesium sulfate }\end{array}$ & $\begin{array}{l}1.30 \\
1.25\end{array}$ & 265 & +112 \\
\hline $\begin{array}{l}\text { Sodium metasilicate plus } \\
\text { magnesium sulfate }\end{array}$ & $\begin{array}{l}2.00 \\
1.25\end{array}$ & 270 & +116 \\
\hline Zinc sulfate & 1.46 & 200 & +60 \\
\hline Nickel sulfate & 1.34 & 170 & +36 \\
\hline Copper sulfate & 0.81 & 170 & +36 \\
\hline Aluminum sulfate & 1.69 & 100 & Negative \\
\hline $\begin{array}{l}\text { Zinc sulfate plus } \\
\text { sodium metasilicate }\end{array}$ & $\begin{array}{l}1.46 \\
1.54\end{array}$ & 210 & +68 \\
\hline $\begin{array}{l}\text { Nickel sulfate plus } \\
\text { sodium metasilicate }\end{array}$ & $\begin{array}{l}1.34 \\
1.54\end{array}$ & 190 & +52 \\
\hline $\begin{array}{l}\text { Copper sulfate plus } \\
\text { sodium metasilicate }\end{array}$ & $\begin{array}{l}0.81 \\
1.54\end{array}$ & 180 & +44 \\
\hline
\end{tabular}

Effectiveness: The additives and/or combination of additives with the plus percentages are more effective than samples treated with the calcium oxide only. Below are the additives which are most effective:

Sodium metasilicate ( 2 percent) plus magnesium sulfate ( 1.25 percent). Sodium metasilicate ( 1.30 percent) plus magnesium sulfate ( 1.25 percent). 
Category*

Lime

Basic Material

Rate of Material

Calcium oxide (lime)

$5 \%$

Cost

Not given

Secondary Material

Magnesium sulfate

1. $25 \%$

Not given

Material Form*

Type of Soil Treated

Mixing

Capability

Solid (lumps)

Clay (Vicksburg)

Good

$\frac{\text { Type of Test }}{\begin{array}{c}\text { Unconfined } \\ \text { compression }\end{array}} \cdot \frac{\begin{array}{c}\text { Purpose of } \\ \text { Material }\end{array}}{\text { Stabilizer }} \cdots \frac{\begin{array}{l}\text { Effective } \\ \text { Strength } \\ \text { Increase }\end{array}}{\text { See comments Excellent }} \cdot \frac{\text { Effectiveness }}{\text { Eng }}$

Total Material Cost

- Per Cu Ft

of Treated Soil

Not given

Test Agency

MIT
Test Report

Reference 41

\section{Comments:}

Treated samples with additives we re compared to samples treated with only 5 percent calcium oxide. Tests were conducted after a 24 hour humid cure followed by a 24 hour water immersion.

The strength of the samples was $235 \mathrm{psi}$. This represents an increase of 88 percent over the strength of the calcium oxide (125 psi) treated samples.

* Basic material 
Category*

Lime

Basic Material

Calcium oxide
Rate of Material

4 and 5\%
Cost

Not given

Secondary Material

Magne sium sulfate

Potassium sulfate

Magnesium chloride

Material Form*

Solid (lumps)
1.0 and $1.25 \%$

$1.25 \%$.

$1.25 \%$

Type of Soil Treated

Clay (Vicksburg)
Not given

Not given

" Mixing

Capability

Good

\begin{tabular}{|c|c|c|c|}
\hline Type of Test & $\begin{array}{c}\text { Purpose of } \\
\text { Material }\end{array}$ & $\begin{array}{l}\text { Effective } \\
\text { Strength } \\
\text { Increase }\end{array}$ & Effectiveness \\
\hline $\begin{array}{l}\overline{\text { Unconfined }} \\
\text { compression }\end{array}$ & Stabilizer & See comments & Excellent \\
\hline
\end{tabular}

Total Material Cost

Per Cu Ft

of Treated Soil

Not given

$\frac{\text { Test Agency }}{\text { MIT }}$

Test Report

Reference 40

\section{Comments:}

Samples with basic material and/or additives were not compared to untreated samples. Tests were conducted after a 24 hour humid cure followed by 24 hours water immersion.

Effectiveness: Calcium oxide ( 5 percent rate) alone was effective in stabilizing the soil (195 psi).

Calcium oxide ( 5 percent rate) with the addition of 1.25 percent magnesium sulfate treated samples had a somewhat higher strength (210 psi).

(Continued on next page)

* Basic material 
Calcium oxide with the other additives gave somewhat lower strengths. 
Category*

Lime

Basic Material

Calcium oxide plus

magnesium sulfate plus

cutback asphalt

Secondary Material

Solvent - gasoline
Rate of Material

$3 \%$

$0.75 \%$
Cost

Not given

\section{2:1 (asphalt, gasoline) Not given}

\begin{tabular}{|c|c|c|}
\hline Material Form* & Type of Soil Treated & $\begin{array}{c}\text { Mixing } \\
\text { Capability } \\
\end{array}$ \\
\hline $\begin{array}{l}\text { Calcium oxide - } \\
\text { Magnesium sulfat } \\
\text { Cutback asphalt - }\end{array}$ & 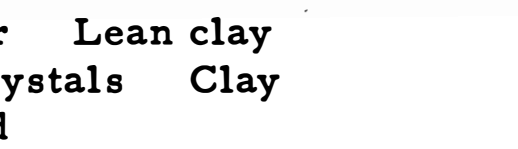 & $\begin{array}{l}\text { Good } \\
\text { Good }\end{array}$ \\
\hline
\end{tabular}

Total Material Cost

Per Cu Ft

$\frac{\text { of Treated Soil }}{\text { Not given }} \cdot \frac{\text { Test Agency }}{\text { WES }} \frac{\text { Test Report }}{\text { Reference 25 }}$

\section{Comments:}

Samples were subjected to 4 days dry cure followed by 4 days wetting by capillary action. Untreated samples after wetting were not suitable for compression tests.

Effectiveness: Lean clay - The samples possessed good compressive strength (191 psi) and the materials waterproofed the samples.

Clay- Same as lean clay except the strength was 188 psi.

* Basic material 
Category*

Lime

Basic Material

Rate of Material

Quicklime

$1-5 \%$

Cost

Not given

Secondary Material

Material Form*

Solid
Type of Soil Treated

Clayey silt, silt, clay, and loess
Mixing

Capability

Good

\begin{tabular}{llll} 
Type of Test & $\begin{array}{c}\text { Purpose of } \\
\text { Material }\end{array}$ & $\begin{array}{l}\text { Effective } \\
\text { Strength } \\
\text { Increase }\end{array}$ & Sffectiveness \\
$\begin{array}{l}\text { Cone } \\
\text { penetrometer }\end{array}$ & Seemments comer & Excellent \\
$\begin{array}{l}\text { Total Material Cost } \\
\text { Per CuFt } \\
\text { of Treated Soil }\end{array}$ & Test Agency & Test Report \\
\hline Not given & MIT & Reference 17
\end{tabular}

Comments:

Quicklime was tested for suitability as a category- I stabilizer-withthe four soils and percentage of treatment below:

(Continued on next page)

* Basic material 


\begin{tabular}{|c|c|c|c|c|}
\hline Soil & $\begin{array}{c}\text { Quicklime } \\
\% \\
\end{array}$ & $\begin{array}{c}\text { Required } \\
\text { Strength } \\
\text { After } \\
2 \text { Hours Cure } \\
\text { psi } \\
\end{array}$ & $\begin{array}{c}\text { Strength of } \\
\text { Treated } \\
\text { Samples } \\
2 \text { Hours Cure } \\
\text { psi } \\
\end{array}$ & $\begin{array}{l}\text { Percent } \\
\text { Strength } \\
\text { Increase } \\
\text { Over } \\
\text { Reguired } \\
\end{array}$ \\
\hline Clayey silt & $\begin{array}{l}1 \\
2 \\
3\end{array}$ & 125 & $\begin{array}{l}210 \\
460 \\
860\end{array}$ & $\begin{array}{r}68 \\
268 \\
588\end{array}$ \\
\hline Silt & 3 & 125 & 230 & 84 \\
\hline Clay & $\begin{array}{l}3 \\
5\end{array}$ & 125 & $\begin{array}{l}170 \\
340\end{array}$ & $\begin{array}{r}36 \\
172\end{array}$ \\
\hline Loess & $\begin{array}{l}1 \\
3 \\
5\end{array}$ & 125 & $\begin{array}{l}160 \\
520 \\
970\end{array}$ & $\begin{array}{r}28 \\
316 \\
670\end{array}$ \\
\hline
\end{tabular}

Effectiveness: All four soils are effectivenly stabilized to meet the requirements of category I stabilization by using 1 to 3 percent Quicklime. 
Category*

Lime

Basic Material

Rate of Material

Cost

Quicklime

3,4 , and $5 \%$

$\$ 1.00$ per $100 \mathrm{lb}$

Secondary Material

Magnesium sulfate

$0.25,0.5,1.0,1.5,2$, and $3 \%$

$\$ 5.00$ per $100 \mathrm{lb}$

Mixing

Material Form*

Type of Soil Treated

Lean clay, heavy clay,

Capability

Powder clayey silt, silt, blue clay, sandy clay, and sand

Good

Effectiveness

$\frac{\text { Type of Test }}{\text { Unconfined }}$

Purpose of

Effective

Material Increase

Stabilizer.

See comments

Excellent except for silt and sand soils

Total Material Cost Per Cu Ft

of Treated Soil

See comments
Test Agency

WES
Test Report

Reference 17

\section{Comments :}

Treated samples were compared to untreated samples. Various combinations were used of the-basic material with the-secondary-material on lean clay. It was found that 4 percent quicklime with 1 percent magnesium sulfate was most effective. This combination was then used in preparing samples with the other soils. Samples were prepared using the Harvard Miniature Compaction Apparatus in five layers (each layer was compacted with ten tamps of a 40-1b spring tamper). After 24 hours cure under 100 percent relative humidity, the samples were tested.

(Continued on next page)

* Basic material 
The strength of all untreated samples was about 20 psi. The increase in the strength of the treated soils (except silt and sand) was sufficient for the 4 percent quicklime and 1 percent magnesium sulfate to be considered as Category 2 stabilizers. Silt and sand treated samples did not meet Category 2 stabilization.

Traffic tests were also conducted on sections of heavy clay and lean clay treated soils. The sections were treated with 4 percent quicklime and 1 percent magnesium sulfate. These sections withstood traffic requirements for emergency military operations.

Costs: A 4 percent quicklime/1 percent magnesium sulfate treatment would cost about $\$ 0.85$ per sq yd (12 in. deep) exclusive of construction or other costs, as the quicklime was about $\$ 1.00$ per $100 \mathrm{lb}$ and sulfate was about $\$ 5.00$ per $100 \mathrm{lb}$. 
Category*

Lime

Basic Material

Rate of Material

Cost

Quicklime

3,5 , and $8 \%$

Not given

Secondary Material

Material Form*

Powder
Type of Soil Treated

Lean clay
Mixing

Capability

Good

\begin{tabular}{|c|c|c|c|}
\hline Type of Test & $\begin{array}{l}\text { Purpose of } \\
\text { Material }\end{array}$ & $\begin{array}{l}\text { Effective } \\
\text { Strength } \\
\text { Increase }\end{array}$ & Effectiveness \\
\hline Unconfined & Stabilizer & See comments & Excellent \\
\hline
\end{tabular}

Total Material Cost

Per Cu Ft

$\frac{\text { of Treated Soil }}{\text { Not given }} \frac{\text { Test Agency }}{\text { WES }} \frac{\text { Test Report }}{\text { Reference } 16}$

\section{Comments:}

Treated samples were compared to untreated samples. Samples were prepared using a Harvard Miniature Compaction Apparatus in five layers (each layer was compacted using ten tamps of a 40-1b spring tamper). After curing for 24 hours under 100 -percent relative humidity, the samples were subjected to unconfined compression tests using the criteria for Category 2 stabilization.

(Continued on next page)

* Basic material 
The test results showed that for between 3 and 8 percent treatment with quicklime, the requirements for Category 2 stabilization were met. Additional tests were conducted with 4 and 8 percent quicklime.

Traffic tests were also conducted. It was found that both 4 and 8 percent quicklime--stabilized soil surfaces are more than adequate for traffic requirements for emergency military roads and airfield operations. 
Category*

Lime

Basic Material

Rate of Material

Cost

Quicklime

4 and 5\%

Not given

Secondary Material

Modifiers: See comments

Material Form*

Powder
Type of Soil Treated

Lean clay

Heavy clay
Mixing

Capability

Good

Good

$\begin{array}{llll}\text { Type of Test } & \begin{array}{l}\text { Purpose of } \\ \text { Material }\end{array} & \begin{array}{l}\text { Effective } \\ \text { Strength } \\ \text { Increase }\end{array} & \text { Effectiveness } \\ \begin{array}{c}\text { Unconfined } \\ \text { compression }\end{array} & \text { Stabilizer } & \text { See comments See comments }\end{array}$

Total Material Cost

Per Cu Ft

of Treated Soil

Not given

Test Agency

Test Report

WES

Reference 18

Comments:

Samples were molded in a Harvard Miniature Compaction Apparatus in five layers (each laye $r^{-}$compacted with ten tamps of a $40^{-}-1 b^{-}$spring tamper). Samples were tested after a 24 hour cure at 100 percent relative humidity and after a 24 -hour cure at 100 percent relative humidity followed by 24 hours water immersion. The strength of the untreated soils was $20 \mathrm{psi}$. Materials which, when added to the soils, helped to increase the strength from 20 to 100 psi or greater were considered to have potential as stabilizers.

(Continued on next page)

* Basic material 
Quicklime ( 5 percent) and quicklime ( 4 percent) plus the following modifiers were tested:

Magnesium sulfate $(1 \%)$

Sodium hydroxide

Magnesium sulfate (1\%) plus alkyl dimethyl benzyl ammonium chloride $(0.5 \%)$

Magnesium sulfate (1.0\%) plus $0.5 \%$ amine $D$ acetate

Magnesium sulfate (1.0\%) plus $0.5 \%$ octadecyl amine acetate Magnesium sulfate $(1 \%)$ plus $0.5 \%$ octadecyl amine
'Magnesium sulfate ( $1 \%$ ) plus $0.1 \%$ n-octylamine

Magne sium sulfate (1\%) plus $1 \%$ sodium or thosilicate

Magne sium sulfate (1\%) plus $1 \%$ sodium metasilicate

Magne sium sulfate (1\%) plus $1 \%$ sodium silicate solution $3 \%$ quicklime plus $0.75 \%$ magnesium sulfate plus $3 \%$ cutback asphalt

Effectiveness: Lean clay - The strength of the dry cured samples of 5 percent quicklime exceeded 100 psi (103); however, the strength after soaking was only $28 \mathrm{psi}$. Several of the samples treated with 4 percent quicklime plus modifier(s) had dry strength in excess of $100 \mathrm{psi}$; however, the wet strengths were much less. The combination of materials which showed the most promise was: 4 percent quicklime plus 1 percent sodium sulfate and 1 percent sodium metasilicate, with 151 psi dry strength and 69 psi after soaking. However, the wet strength did not meet the criteria of $100 \mathrm{psi}$.

Heavy clay - The strength of the dry cure samples of 4 percent quicklime plus 1 percent magne sium sulfate was 132 psi; however, the strength after soak was only $48 \mathrm{psi}$ (which does not meet the required minimum of $100 \mathrm{psi}$ ). 
Category: Resin 


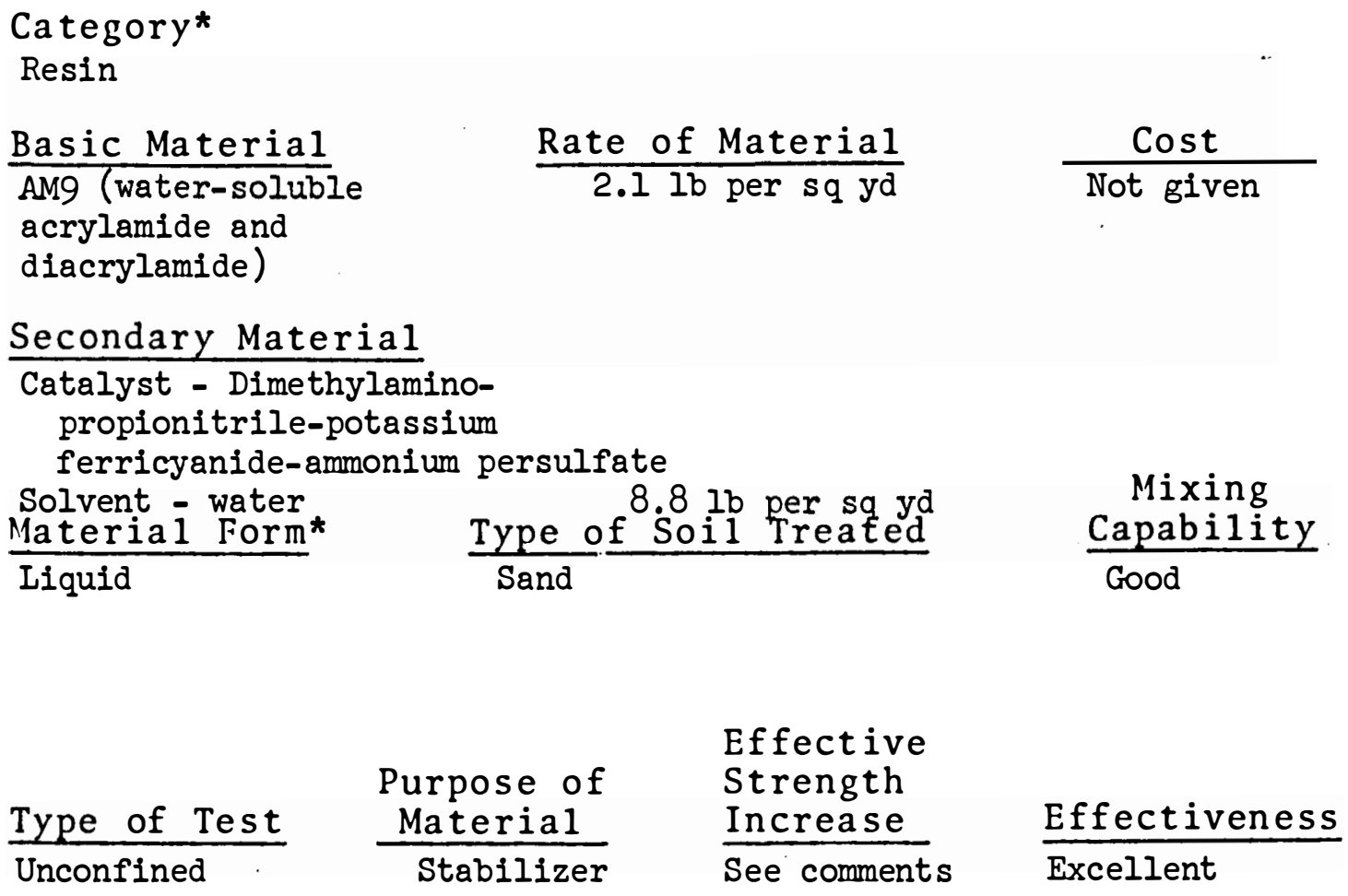

Total Material Cost Per Cu Ft $\frac{\text { of Treated Soil }}{\text { Not given }} \quad \frac{\text { Test Agency }}{\text { Ashland Chemical Co. }} \frac{\text { Test Report }}{\text { Reference } 57}$

Comments:

Treated samples were not compared to untreated samples. Cure time was 3 days at room termperature. Unconfined compression strength was 1723 psi. After wet-dry ( 8 cycles), unconfined compression strength was 1180 psi. Wet-dry cycles consisted of water immersion of samples for 8 hours at room temperature, water drained off, and then samples were subjected, to heat for 16 hours in a forced draft oven at $140 \%$.

\footnotetext{
* Basic material
} 
Cat egory*

Resin

Basic Material

$\underline{\text { Rate of Material }}$

Cost

Aniline-furfural

Secondary Material

$$
\begin{aligned}
& 3.3 \% \\
& (2.1 \% \text { aniline and } \\
& 1.2 \% \text { furfural) }
\end{aligned}
$$

Not given

Mixing

$\frac{\text { Capability }}{\text { Good }}$

\begin{tabular}{|c|c|c|c|}
\hline Type of Test & $\begin{array}{c}\text { Purpose of } \\
\text { Material }\end{array}$ & $\begin{array}{l}\text { Effective } \\
\text { Strength } \\
\text { Increase } \\
\end{array}$ & Effectiveness \\
\hline $\begin{array}{l}\text { Unconfined } \\
\text { compression }\end{array}$ & $\begin{array}{l}\text { Stabilizer } \\
\text { Waterproofer }\end{array}$ & $696 \%$ & Excellent \\
\hline
\end{tabular}

$\frac{\text { Material Form* }}{\text { Liquid }}$ $\frac{\text { Type of Soil Treated }}{\text { Loess }}$

Total Material Cost

Per $\mathrm{Cu} \mathrm{Ft}$

of Treated Soil

Not given

Test Agency

WES
Test Report

Reference 24

\section{Comments:}

Treated samples were compared to untreated samples (23 psi unconfined compression strength). Samples prior to tests were air-dried for 4 days followed by 4 days wetting by permeation. The strength of the treated samples was $183 \mathrm{psi}$ which was an increase of 696 percent. This material showed potential as a waterproofer.

This material was also subjected to field investigations at the WES as a dustproofer and waterproofer. The results indicated that further investigation was warranted.

* Basic material 
Category*

Resin

Basic Material

Rate of Material

Cost

Aniline furfural

Aniline - 2\%

Aniline

Furfural - 1\%

(\$0.16 per $1 b)$

Furfural

Secondary Material

$(\$ 0.18$ per $1 \mathrm{~b})$

Material Form*

$\frac{\text { Type of Soil Treated }}{\text { Lean clay }}$

Mixing

Liquid

Clay

Capability

Effectiveness

Type of Test

Purpose of

Effective

Increase

Unconfined

compression

Stabilizer

See comments

Excellent

and traffic

Waterproofer

Dustproofer

Total Material Cost

Per Cu Ft

$\frac{\text { of Treated Soil }}{\$ 1.18(1969 \text { cost })}$

Test Agency

WES

Test Report

Reference 51

\section{Comments :}

Samples for the laboratory tests were molded in a Harvard Miniature Compaction. Apparatus. After the samples were taken from the molds, they were air-aried for 4 days followed by wetting cycles by capillary action for 4 days. This completed one cycle. Four cycles were completed prior to sample testing.

Analine furfural proved to be a highly effective waterproofing agent. Numerous ratios and percentages of aniline to furfural were used in determining the most effective combination. The rate given above proved to be all round the most effective.

* Basic material 


\section{Category* \\ Resin}

Basic Material

Aropol 7110
Rate of Material

$$
2.6,6.0,6.5
$$

and $8.7 \mathrm{lb}$ per sq jd

Secondary Material

Solvent - styrene

Material Form*

Iiquid
15, 11.8, 15.5 and $15.4 \mathrm{lb}$ per sq yd

Type of Soil Treated Sand
Not given

Mixing

Capability

Good

\begin{tabular}{|c|c|c|c|}
\hline Type of Test & $\begin{array}{c}\text { Purpose of } \\
\text { Material }\end{array}$ & $\begin{array}{l}\text { Effective } \\
\text { Strength } \\
\text { Increase } \\
\end{array}$ & Effectiveness \\
\hline $\begin{array}{l}\text { Unconfined } \\
\text { compression }\end{array}$ & Stabilizer & See comments & Excellent \\
\hline
\end{tabular}

Total Material Cost

Per Cu Ft

$\frac{\text { of Treated Soil }}{\text { Not given }} \quad \frac{\text { Test Agency }}{\text { Ashland Chemical Co. }} \frac{\text { Test Report }}{\text { Reference } 57}$

Comments:

Treated samples were not compared to untreated samples. Samples were cured for three days at room temperature. Strengths for $2.6 \mathrm{lb}$ per sq yd with $15 \mathrm{lb}$ per sq yd solvent and $8.7 \mathrm{lb}$ per sq yd solvent were 1173 and 1890 psi, respectively. After 8 wet-dry cycles, these strengths were 1412 and 2020 psi. Each wet-dry cycle consisted of immersing the samples in water for 8 hours, pouring off water, and then subjecting the samples to heat for 16 hours in a forced draft oven at $140 \mathrm{~F}$.

* Basic material 
Category*

Resin

$\frac{\text { Basic Material }}{\text { Arothane } 170}$

$\frac{\text { Rate of Material }}{4 \%}$

Cost

Not given

Secondary Material

Solvent - butyl acetate $\quad 3 \% \quad$ Not given

Material Form*

$\frac{\text { Type of Soil Treated }}{\text { Sand }}$

Mixing

Capability

Good

Effectiveness

Type Purpose of Strength

Type of Test
$\begin{aligned} & \text { Unconfined } \\ & \text { compression }\end{aligned}$
Totab

Per $\mathrm{Cu} \mathrm{Ft}$

of Treated Soil

Test Agency

Test Report

Not given

Ashland Chemical Co. Reference 57

Comments:

Treated samples were not compared to untreated samples. Strength after 3 days cure at room temperature was 706 psi. After 8 wet-dry cycles, the strength was $667 \mathrm{psi}$. Each cycle consisted of immersing the samples in water for 8 hours, pouring water off, and subjecting the samples to heat for 16 hours in a forced draft oven at $140 \mathrm{~F}$.

* Basic material 

Category*
Resin
Basic Material
Bisphenol A
$\frac{\text { Rate of Material }}{1.3,2.6,5.2,6.9}$
(Epon 828)
and $\mathrm{ll} \mathrm{lb}$ per sq yd
$\frac{\text { Cost }}{\text { Not given }}$

Secondary Material

Catalyst - Ashland \#1496 Included with basic material

Solvent - solox

$5.1,10.4,14.6$,

and $16.4 \mathrm{lb}$ per $\mathrm{sq}$ yd

Material Form*

Type of Soil Treated

Mixing

Capability

liquid

Sand

Good

$\frac{\text { Type of Test }}{\begin{array}{c}\text { Unconfined } \\ \text { compression }\end{array}} \quad \frac{\begin{array}{l}\text { Purpose of } \\ \text { Material }\end{array}}{\text { Stabilizer }} \quad \frac{\begin{array}{l}\text { Effective } \\ \text { Strength } \\ \text { Increase }\end{array}}{\text { See comments }} \quad \frac{\text { Effectiveness }}{\text { Excellent }}$

Total Material Cost

Per $\mathrm{Cu} \mathrm{Ft}$

of Treated Soil

Not given

Test Agency

Ashland Chemical Co.
Test Report

Reference 57

\section{Comments:}

Treated samples not compared to untreated samples. A strength of 1079 psi was achieved as a use level of $5.2 \mathrm{lb}$ per sq yd resin and 5.1 per sq yd solvent. This strength was achieved after three days cure. Wet-dry resistance was determined by immersing the specimens in water for eight hours at room temperature, draining the water, and subjecting them to heat for 16 hours in a forced draft oven at $140 \mathrm{~F}$. After eight cycles, they were subjected to unconfined compression tests. The strength of the specimens at the rate given above was 1140 psi.

* Basic material 
Category*

Resin

Basic Material

Calcium acrylate $\frac{\text { Cost }}{\text { Not given }}$

Mixing

Capability

Good

$\frac{\text { Material Form* }}{\text { Powder }} \cdot \frac{\text { Type of Soil Treated }}{\text { Loess }} \quad \frac{\text { Capability }}{\text { Good }}$

\begin{tabular}{|c|c|c|c|}
\hline Type of Test & $\begin{array}{c}\text { Purpose of } \\
\text { Material }\end{array}$ & $\begin{array}{l}\text { Effective } \\
\text { Strength } \\
\text { Increase }\end{array}$ & Effectiveness \\
\hline $\begin{array}{l}\text { Unconfined } \\
\text { compression }\end{array}$ & $\begin{array}{c}\text { Stabilizer } \\
\text { Dustproofer }\end{array}$ & $408 \%$ & Excellent \\
\hline
\end{tabular}

Total Material Cost

Per Cu Ft

of Treated Soil

Not given

$\frac{\text { Test Agency }}{\text { WES }}$

$\frac{\text { Test Report }}{\text { Reference } 24}$

\section{Comments:}

Treated samples were compared to untreated samples (23 psi unconfined compression strength). Samples prior to tests were air-dried for 4 days followed by 4 days wetting by permeation. The strength of the treated samples was 117 psi which was an increase of 408 percent. This material showed potential as a waterproofer.

This material was also subjected to field investigations at the WES as a dustproofer and waterproofer. The results did not indicate that further work with this material should be concucted.

\footnotetext{
* Basic material
} 
Category*

Resin

Basic Material

Rate of Material

Cost

Calcium acrylate

Varied

Not given

Secondary Material

See comments for catalysts

and activators

Material Form*

Powder

$\frac{\text { Type of Soil Treated }}{\text { Sandy clay }}$

Mixing

Capability

Effectiveness

Type of Test

Purpose of

Effective

Material

Strength

Tensile

Stabilizer

Increase

See comments

See comments

Total Material Cost

Per Cu Ft

of Treated Soil

Test Agency

Test Report

Not given

MIT

Reference 31

Comments:

Treated samples were not compared to untreated samples. A series of soil-calcium acrylate solutions with a pH range of 3.7 to 9.9 were studied. It was found that as the $\mathrm{pH}$ increased, the tensile strength and flexibility increased. Various inhibitors, activators, and

catalysts used with calcium acrylate are shown in the following table:

(Continued on next page)

* Basic material 
Inhibitors

Benzoquinone

Hydroquinone

Picric acid

Methylene blue
Activators

\section{Sodium theiosulfate \\ Sodium sulfite \\ Sodium bisulfite \\ Sodium hydrosulfite}

Sodium sulfide

Potassium ferrocyanide

Ferrous sulfate

Silver nitride

Stannous chloride

Cuprous chloride

Cupric sulfate

Titanium sulfatein

Hydrochloric acid

Hydroxylamine hydrochloride

Hydrazine hydrate

Hydrazine sulfate

Hydroquinone

Catechol

Resorcinol

Phloroglucinol

Dextrose

Tetramethylene pentamine
Catalysts
Ammonium pursulfate
Potassium persulfate
Hydrogen peroxide
Sodium pyrophosphate peroxide
Sodium carbonate peroxide
Sodium perborsilicate
Calcium peroxide
Urea peroxide
t-butyl hydroperoxide
l-hydroxycyclohexyl-
hydroperoxide-l

The properties of a soil stabilized by the in-situ polymerization of calcium acrylate depend on the method of polymerization. The type of redox system used has the most influence. Three satisfactory redox systems were found: armonium persulfate-sodium thiosulfate, potassium persulfate-sodium thiosulfate, and t-butyl hydroperoxide-sodium thiosulfate. 


\section{Category*}

Resin

Basic Material

Rate of Material

Calcium acrylate

Varied

Cost

Not given

Secondary Material

Salt additives (below)

Varied

Material Form*

Powder

Type of Soil Treated

Sandy clay

Effective

Purpose of Strength

Type of Test

Material

Increase

Effectiveness

Tensile

Stabilizer

See comments

Total Material Cost

Per Cu Ft

of Treated Soil

Not given

Test Agency

$\frac{\text { Test Report }}{\text { Reference } 31}$

Comments:

Treated samples not compared to untreated samples.

a. Ten of the salts tested are -- ammonium, lithium, sodium, magnesium, manganese, and nickel chlorides, and sodium, magnesium, manganese, and nickel sulfates-- had minor effects on the tensile strength.

b. Two salts, calcium chloride and aluminum chloride, increased the tensile strength at the highest ratios.

c. Three salts, zinc chloride, zinc sulfate, and chromium chloride, increased the tensile strength markedly.

* Basic material 
Category*

Resin

Basic Material

$\frac{\text { Rate of Material }}{\text { Varied }}$

Cost

Calcium acrylate

Not given

Secondary Material

Various salts (see comments)

Material Form*

Powder

Type of Soil Treated

Sandy clay

Mixing

Capability

Good

Type of Test $\quad \begin{array}{lll}\begin{array}{c}\text { Purpose of } \\ \text { Material }\end{array} & \begin{array}{l}\text { Effective } \\ \text { Ttrength } \\ \text { Increase }\end{array} & \text { Effectiveness } \\ \text { Stabilizer } & \text { See comments } & \text { See comments }\end{array}$

Total Material Cost

Per Cu Ft

of Treated Soil

Not given

\section{Comments:}

Various salts tested with calcium acrylate are given below. No strength values were given; however, a work-description of the test results was given on each salt tested.

Ammonium chloride - No appreciable effect on the strength of samples.'

Lithium chloride - No appreciable effect on the strength of samples.

Sodium chloride - No appreciable effect on the strength of samples (Continued on next page)

* Basic material 
Sodium sulfate - No effect on the tensile strength, however, the elongation was increased with increasing amounts of sulfate.

Potassium chloride - Prevented solidification of samples.

Barium chloride - Prevented solidification of" samples.

Copper sulfate - Prevented solidification of samples.

Ferric chloride - Prevented solidification of samples

Lead acetate - Prevented solidification of samples.

Magnesiom chloride - No appreciable effect on strength of samples.

Magnesium sulfate - No appreciable effect on strength of samples.

Nickel chloride - No appreciable effect on strength of samples.

Nickel sulfate - No apprecialbe effect on strength of samples.

Manganous chloride - No effect on tensile strength; however, the elongation decreased.

Manganous sulfate - Slight increase in tensile strength and a -. slight decrease in elongation.

Zinc chloride - Slight increase in tensile strength and a great increase in elongation.

Zinc sulfate - Increased tensile strength, decreased elongation, and samples brittle.

Aluminum sulfate - Increased tensile strength, decreased elongation, and samples brittle.

Chromium chloride - Increased tensile strength, decreased elongation, and samples brittle. 
Category*

Resin

Basic Material

Rate of Material

Cost

Epon VIII

$20 \%$

Not given

Secondary Material

Curing agents

Agent A (amine)

$10 \%$

Not given

Diethylenetriamine

(see comments)

Water

$10 \%$

Not given

35 to $40 \%$

Material Form*

Type of Soil Treated

Liquid

Sandy clay

Mixing

Capability

Good

\begin{tabular}{|c|c|c|c|}
\hline Type of Test & $\begin{array}{l}\text { Purpose of } \\
\text { Material }\end{array}$ & $\begin{array}{l}\text { Effective } \\
\text { Strength } \\
\text { Increase }\end{array}$ & Effectiveness \\
\hline Tensile & Stabilizer & See comments & None \\
\hline
\end{tabular}

Total Material Cost

Per Cu Ft

of Trea

Treated samples were not compared to untreated samples.

a. Agent A (amine). After 4 hours curing time in an oven at $110^{\circ} \mathrm{C}$, tensile strength of 410 psi for the dry samples was obtained. After soak tests, the strength dropped to 220 psi.

(Continued on next page)

* Basic material 
ob. Diethylenetriamine. After 4 hours curing time in an oven at $110^{\circ} \mathrm{C}$, tensile strength of 400 psi for the dry samples was obtained. After soak tests, the strength dropped to 210 psi.

Samples treated with materials that have to oven cure are impractical for field use. 
Category*

Resin

Basic Material

Epon 562

Secondary Material

$70 \%$ diethylene triamin

$30 \%$ dimethyl aminomethyl phenol

(above 2 are curing agents)

Acetone (solvent)

Potassium hydroxide (KOH)

Rate of Material

$10 \%$

$2 \%$

$2 \%$

$10 \%$

$1 \%$

Material Form*

Liquid

Type of Soil Treated

Sandy clay

Purpose of

Type of Test

Tensile
Effective

Strength

Increase

See comments
Not given

Not given

Not given

Not given
Mixing Capability Good

Total Material Cost

Per $\mathrm{Cu} \mathrm{Ft}$

of Treated Soil
Not given

Test Agency

Test Report

See comments

Comments:

The samples where acetone was used as a solvent were compared to samples treated with resin only.

Effectiveness: For the same period of cure time, the samples with the solvent had an increase in tensile strength of 46 percent. Therefore, the solvent is effective for achieving a faster cure rate.

The potassium hydroxide when used with Epon 562 caused a detrimental effect on the strength of the samples.

* Basic material 
Category*

Resin

Basic Material

Rate of Material

Epon 828

$10 \%$ based on wight of dry
soil

Cost

Not given

Secondary Material

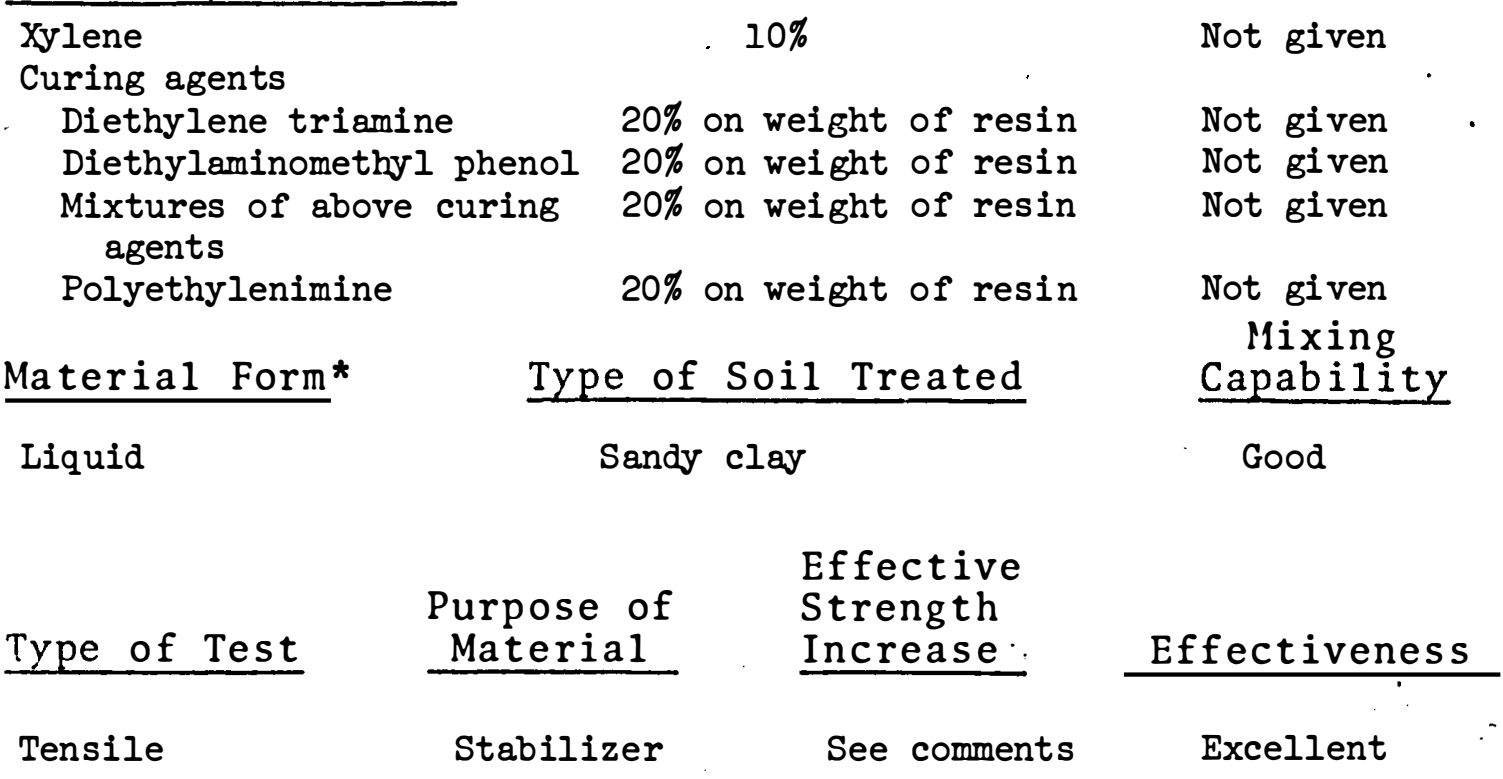

Total Material Cost

Per $\mathrm{Cu} F t$

of Treated Soil

Not given

Test Agency

Test Report

Comments:

Treated samples not compared to untreated samples. Curing agent, diethylene triamine, when used in preparing test samples, yielded soils with dry and rewet tensile strengths (160 to $200 \mathrm{psi}$ ); however, these systems do not develop high strength on curing under wet conditions. These strengths were developed only after one to six days cure time. Diethylaminomethyl phenol as a curing agent yielded soil of low dry and rewet strength ( 40 and $3 \mathrm{psi}$ ) but developed somewhat higher strength of $80 \mathrm{psi}$, rewet of $70 \mathrm{psi}$, and also $80 \mathrm{psi}$ strength on curing under wet conditions. The use of polyethyleneimine gave poor results when used as a curing agent.

* Basic material 
Category*

Resin

Basic Material

Epon 828
Rate of Material

$10 \%$ of dry soil weight
Cost

Not given

Secondary Material

$70 \%$ diethylene triamine

$30 \%$ dimethyl aminomethyl phenol

(curing agents)

Solvents - see comments
$2 \%$ on dry soil Not given weight
Material Form*

Liquid
Type of Soil Treated

Sandy clay
Mixing Capability

Good

$\begin{array}{llll}\text { Type of Test } & \begin{array}{l}\text { Purpose of } \\ \text { Material }\end{array} & \begin{array}{l}\text { Effective } \\ \text { Strength } \\ \text { Increase }\end{array} & \text { Effectiveness } \\ & \text { Stabilizer } & \text { See comments } & \text { See comments }\end{array}$

Total Material Cost Per Cu Ft

of Treated Soil

Not given
Test Agency

MIT
Test Report

Reference 37

Comments:

Solvents used were acetone ( 1 to 3 percent) and zylene ( 1 percent). These were used separately with the basic material and secondary materials. The treated samples where the solvent was used were compared to samples treated with the resin only.

Effectiveness: The samples where the xylene was used had less tensile strength than those treated with only the resin.

The acetone accelerated the curing of the samples. As compared to (Continued an next page)

* Basic material 
samples treated with only the resin and after one day cure time and 24 hours water imersion, the samples treated with acetone had a strength increase of 66 percent. 
Category*

Resin

Basic Material $\quad$ Rate of Material

Epon 828

3,5 , and $10 \%$

Cost

Not given

Secondary Material

Curing agent: $7: 1$ ratio of $20 \% \quad$ Not given

diethylene triamine to

dimethyl aminomethy

phonel

Material Form*

Liquid

$\frac{\text { Type of Test }}{\begin{array}{c}\text { Unconfined } \\ \text { compression }\end{array}}$

Type of Soil Treated

Lean clay and heavy clay
Mixing

Capability

Good

Total Material Cost

Per Cu Ft

$\frac{\text { of Treated Soil }}{\text { Not given }} \frac{\text { Test Agency }}{\text { WES }}$

Effective

Purpose of Strength

Material Increase

Stabilizer

See corments

Effectiveness

None

Test Report

Internal Data

(1956-57), not published

\section{Comments:}

Treated samples were compared to untreated samples. Preparation of samples was with a Harvard miniature compaction apparatus, five layers, ten tamps per layer with a 20-lb spring tamper. The samples were tested against $C$ Category 1 stabilization requirements.

Effectiveness: The strength increase of the treated samples as compared to the untreated samples varied from 400 to 600 percent; however, this did not satisfy the requirements.

* Basic material 
Category*

Resin

Basic Material

Epon 828
Rate of Material $20 \%$
Cost

Not given

Secondary Material

Curing agents

Tetraethylenepentamine

Diethylenetriamine

Water (See comments)

$10 \%$

10 and $15 \%$

35 and $40 \%$

Not given

Not given

Mixing

Type of Soil Treated

Capability

Good

Liquid

Sandy clay

Effectiveness

Type of Test

Purpose of

Effective

Tensile

Stabilizer

Increase

See comments

See comments

Total Material Cost

Per Cu Ft

$\frac{\text { of Treated Soil }}{\text { Not given }} \frac{\text { Test Agency }}{\text { MIT }} \frac{\text { Test Report }}{\text { Reference } 34}$

Comments:

These samples were cured at room temperatur.

a. Tetraethylenepentamine. Very low tensile strengths were developed after a long curing time of seven days with this curing agent. Effectinveness - None.

b. Diethylenetriamine. Relatively high tensile strengths were developed ( 395 to 530 psi with the different rates of the curing agent) after long curing times of 7 to 12 days. The samples after the soak tests retained most of the dry cure strength. Effectiveness - Moderate. (Continued on next page)

* Basic material 
Other curing agents were used with Apon 834 at rates which varied from 6 to 67 percent, depending on which agent was used with 834 . Long curing times from three to seven days were required on drycured samples and from two to seven days on wet-cured samples. The dry-cured samples had good tensile strengths; however, they were poor after the soak test. Agents used in the dry-cured samples were diethylenetriamine, monothanolamine, benzylamine, hexamethylenediamine, citric acid, polyamide 115, dimethylamincmethylphenol, and 2,4,6tridimethylaminomethylphonel.

Agents used in the wet-cured samples were citric acid, diethylenetriamine, polyamide 115, and dimethylaminomethylphenol. The strength of the wet-cured samples was poor even after two to seven days of cure time. 
Category: Salt 
Category*

Salt

Basic Material

Arquad 2HT

(Dialkyl dimethyl-

ammonium chloride)

Secondary Material
Rate of Material

$0.5 \%$
Cost

Not given
Mixing

Capability

Good

Paste

Type of Soil Treated

Loes 8
Effectiveness

Excellent $\frac{\text { Type of Test }}{\text { Unconfined }}$ compression

$\begin{aligned} & \text { Purpose of } \\ & \text { Material }\end{aligned}$
$\begin{aligned} & \text { Stabilizer } \\ & \text { Waterproofer }\end{aligned}$

Effective Strength

Increase $374 \%$
Test Agency WES
Test Report

Reference 24

\section{Comments:}

Treated samples were compared to untreated samples (23 psi strength). Samples prior to tests were air-dried for 4 days followed by 4 days wetting by permeation. The strength of the treated samples was 109 psi which was an increase of 374 percent. This material showed potential as a waterproofer.

This material was also subjected to field investigations as a waterproofer and dustproofer at WES and the results indicated that further tests of this material were warranted.

* Basic material 


\section{Category: Silicate}

Al81 
Category*

Silicate

Basic Material

Rate of Material

Cost

Sodium silicate $(30 \%$

$14.5 \%$

Not given

solution)

Secondary Material

Mixing.

Capability

Good

Effectiveness

Effective

Purpose of Strength

Type of Test

Unconfined

Material

Increase

$243 \%$

Excellent

compression Waterproofer

Total Material Cost

Per $\mathrm{Cu} \mathrm{Ft}$

of Treated Soil

Not given

Test Agency

WES
Test Report

Reference 24

\section{Comments:}

Treated samples were compared to untreated samples (23 psi unconfined compression strength). Samples prior to tests were aiddried for 4 days followed by 4 days wetting by permeation. The strength of the treated samples was 79 psi which was a 243 percent increase. This material showed some potential as a waterproofer.

This material was also subjected to field investigations at the WES as a waterproofer and dustproofer. The results indicated that no further tests were warranted.

* Basic material 
Category*

Silicate/Other

Basic Material

Rate of Material

Cost

Sodium silicate plus

Varied (see comments) Not given

basic magnesium carbonate

Secondary Material

Material Form*

Powder plus powder
Type of Soil Treated

Loess
Mixing

Capability

Good

\begin{tabular}{|c|c|c|c|}
\hline Type of Test & $\begin{array}{l}\text { Purpose of } \\
\text { Material } \\
\end{array}$ & $\begin{array}{l}\text { Effective } \\
\text { Strength } \\
\text { Increase } \\
\end{array}$ & Effectiveness \\
\hline $\begin{array}{l}\text { Unconfined } \\
\text { compression }\end{array}$ & Stabilizer & See comments & Excellent \\
\hline
\end{tabular}

Total Material Cost

Per Cu Ft

of Treated Soil

Not given

Test Agency

MIT
Test Report

Reference 40

\section{Comments:}

Samples treated with basic materials were not compared to untreated samples. (Sclium silicate is a combination of silicon dioxide and sodium oxide.)

The effects of varying the silica and magnesium contents were studied. For each test, two of the components were held at the same rate while the rate of the third one varied.

(Continued on next page)

* Basic material 


\section{Effectiveness:}

Silica content varied. $2.51,3.82$, and 5.12 percent with magnesium ( 1.80 percent) and sodium (1.59 percent) constant. Highest strength achieved was 140 psi at 3.82 percent silica.

Magnesium content varied. $1.20,1.80,2.40$, and 3.00 percent with silica (5.12 percent) and sodium (1.59 percent) constant. Highest strength achieved was 105 psi at 3.00 percent magnesium.

The most effective combination for stabilization was 3.82 percent silica, 1.59 percent sodium, and 1.80 percent magnesium - 140 psi. 
Category*

Silicate

Basic Material Rate of Material

Cost

Sodium silicate $\mathrm{N} \quad \cdot \quad 21.6 \%$

Not given

Secondary Material

Solvent - water $3 \%$

Material Form*

Type of Soil Treated

Liquid

Sand

Mixing

Capability

Good

$\begin{array}{llll}\text { Type of Test } & \begin{array}{l}\text { Purpose of } \\ \text { Material }\end{array} & \begin{array}{l}\text { Effective } \\ \text { Strength } \\ \text { Increase }\end{array} & \text { Snconfined }\end{array} \quad \frac{\text { Effectiveness }}{\text { See comments }}$

Total Material Cost

Per $\mathrm{Cu} \mathrm{Ft}$

$\frac{\text { of Treated Soil }}{\text { Not given }} \frac{\text { Test Agency }}{\text { Ashland Chemical Co. Reference } 57}$

Comments :

Treated samples were not compared to untreated samples. Initial tests were conducted after three days cure at room temperature. Strength was 650 psi, After the 8 wet-dry cycles, the strength dropped to $240 \mathrm{psi}$. Each wet-dry cycle consisted of immersion of the samples in water for 8 hours, pouring off the water, and drying for 16 hours in a forced draft oven at $140^{\circ} \mathrm{F}$.

* Basic material 
Category*

Silicate

Basic Material Rate of Material

Sodium silicate (composed $1.59 \%$ sodium oxide

of two components at

$3.82 \%$ silicon dioxide

right)

Secondary Material

Precipitating agents:

Magne sium oxide

Magne sium carbonate

$0.77,1.03$, and $1.54 \%$

1.2 and $1.8 \%$

Material Form*

Type of Soil Treated

Powder

Clayey silt

Not given

"Mixing

Capability

Good

\begin{tabular}{|c|c|c|c|}
\hline vpe of Test & $\begin{array}{l}\text { Purpose of } \\
\text { Material }\end{array}$ & $\begin{array}{l}\text { Effective } \\
\text { Strength } \\
\text { Increase }\end{array}$ & Effectiveness \\
\hline Unconfined & Stabilizer & See comments & Excellent \\
\hline
\end{tabular}

Total Material Cost

Per Cu Ft

$\frac{\text { of Treated Soil }}{\text { Not given }} \frac{\text { Test Agency }}{\text { MIT }} \frac{\text { Test Report }}{\text { Reference } 40}$

Comments:

Samples treated with each precipitating agent were not compared to samples without treatment. Tests were conducted after one day humid cure plus one day water immersion.

(Continued on next page)

* Basic material 
Effectiveness: The basic material with 1.8 percent magnesium carbonate was the most effective stabilizer (650 psi).

All rates of each agent we re effective in stabilizing the soil. Magnesium oxide ( 1.54 percent) gave the highest strength with this agent only.

A combination of the two, 1.2 percent magnesium carbonate plus 0.26 percent magnesium oxide, gave a strength of 565 psi.

The reaction of magnesium oxide is very slow; however, it has three advantages over magnesium carbonate: (1) smalle $r$ weight must be added to the soil per equivalent of magne sium, (2) magnesium oxide is more dense and less bulky for a given weight, and (3) the carbonate ion is not present in the oxide and the problem of possible sodium carbonate crystallization is eliminated. 


\section{Category*}

Silicate

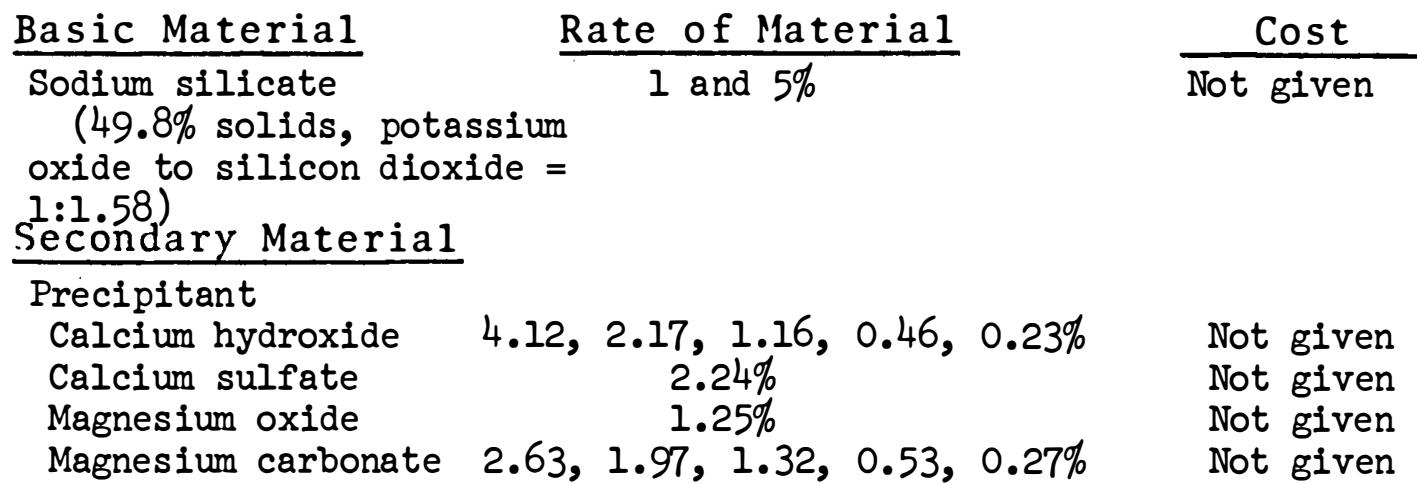

Material Form

Type of Soil Treated

Mixing

Capability

White lumps or powder Clayey silt

Good

\begin{tabular}{|c|c|c|c|}
\hline Type of Test & $\begin{array}{c}\text { Purpose of } \\
\text { Material }\end{array}$ & $\begin{array}{l}\text { Effective } \\
\text { Strength } \\
\text { Increase }\end{array}$ & Effectiveness \\
\hline $\begin{array}{l}\text { Unconfined } \\
\text { compression }\end{array}$ & Stabilizer & See comments & Excellent \\
\hline
\end{tabular}

Total Material Cost

Per Cu Ft

$\frac{\text { of Treated Soil }}{\text { Not given }}$

Test Agency

$\frac{\text { Test Report }}{\text { Reference } 39}$

\section{Comments:}

Samples treated with each precipitant were not compared to samples treated with basic material only. Samples were tested in various combinations (percent) with basic material and precipitants. The most promising based on 24 hours humid cure strength are given in order of effectiveness :

(Continued on next page)

* Basic material 


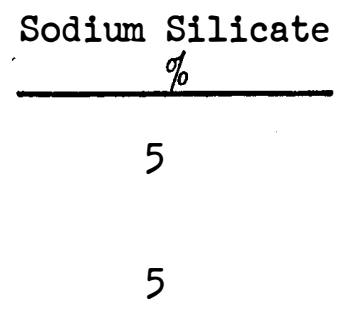

24 Hours Hurnid Cure

Precipitant (\%) Compressive Strength, psi

Magnesium - $1.97 \quad 490$

carbonate

Calcium - 4.12

282

hydroxide 
Category*

Silicate

Basic Material

Rate of Material

See comments

See comments

Magne sium carbonate

(precipitant)

Material Form*

White lumps or powder

Secondary Material
Cost

Not given

Not given

Mixing

Capability

Good

\begin{tabular}{|c|c|c|c|}
\hline Type of Test & $\begin{array}{l}\text { Purpose of } \\
\text { Material }\end{array}$ & $\begin{array}{l}\text { Effective } \\
\text { Strength } \\
\text { Increase }\end{array}$ & Effectiveness \\
\hline $\begin{array}{l}\text { Unconfined } \\
\text { compression }\end{array}$ & Stabilizer & See comments & Excellent \\
\hline
\end{tabular}

Total Material Cost

Per Cu Ft

$\frac{\text { of Treated Soil }}{\text { Not given }} \quad \frac{\text { Test Agency }}{\text { MIT }} \frac{\text { Test Report }}{\text { Reference 39 }}$

Comments:

Tests were run to dete mine the effect of varying the amount of silicon dioxide in the basic material and varying the amount of magnesium carbonate. A ratio of $1: 2$ and $1: 1.58$ sodium oxide $\left(\mathrm{Na}_{2}{ }^{\mathrm{J}}\right)$ to silicon

dioxide $\left(\mathrm{SiO}_{2}\right)$ was used with equivalent $\mathrm{Mg}++$ per $100 \mathrm{gm}$ dry soil of $0.0308,0.0462$, and 0.0615 .

Effectiveness: The most effective ratio of $\mathrm{Na}_{2} 0: \mathrm{SiO}_{2}$ was $1: 2$ and equivalent $\mathrm{Mg}++$ was 0.0462 . The compressive strength of this combination of basic material and precipitant was very high after 24 hours humid cure followed by 24 hours water immersion - 665 psi.

* Basic material 
Category*

Silicate

Basic Material

(49.8\% solids; sodium

$\frac{\text { Rate of Material }}{5 \%}$

oxide to silicon dioxide $=$

$1: 1.58)$

Secondary Material

Precipitant - Magnesium carbonate $1.97 \%$

Waterproofing agents:

Octylamine

Arquad 12 (lauryl trimethyl

ammonium chloride)

Material Form*

Type of Soil Treated

White lumps or powder Clayey silt

$0.1 \%$

$0.1 \%$

Not given
Not given $\frac{\text { Type of Test }}{\text { Unconfined }}$ compression
Purpose of Material

Stabilizer
Effective

Strength

Increase

See comments
Mixing

Capability

Good

Total Material Cost

Per Cu Ft

\section{of Treated Soil}

Not given
Test Agency

MIT
Test Report

Reference 39

Comments:

Samples treated with each waterproofing agent were compared to samples treated with precipitant and basic material. Samples were cured for 24 hours and immersed in water for 24 hours then tested.

(Continued on next page)

* Basic material 


\begin{tabular}{|c|c|c|c|}
\hline Precipitant $(\%)$ & $\begin{array}{c}\text { Waterproofing Agent } \\
\%\end{array}$ & $\begin{array}{l}\text { Strength } \\
\text { psi }\end{array}$ & $\begin{array}{c}\text { Strength Change } \\
\text { Based on Treated } \\
\text { Samples Without } \\
\text { Waterproofing } \\
\text { Agent } \\
\% \\
\end{array}$ \\
\hline $\begin{array}{l}\text { Magnesium carbonate } \\
(1.97)\end{array}$ & None $(0)$ & 380 & -- \\
\hline $\begin{array}{l}\text { Magnesium carbonate } \\
(1.97)\end{array}$ & Octylamine $(0.10)$ & 417 & +10.0 \\
\hline $\begin{array}{l}\text { Magnesium carbonate } \\
(1.97)\end{array}$ & Arquad $12(0.10)$ & 452 & +19.0 \\
\hline
\end{tabular}

Effectiveness: The 24 hours humid cure strength of the magnesiumcarbonate-treated samples was 490 psi. After 24 hours water immersion, the strength was $380 \mathrm{psi}$. This is a dropoff of 22 percent without a waterproofing agent. From these data listed above, the addition of the waterproofing agents had little effect on improving the strength of the samples. 
Category*

Silicate /Other

Basic Material

Rate of Material

Sodium silicate plus

Sodium oxide - $1.6 \%$

calcium hydroxide, $\mathrm{Ca}(\mathrm{OH})$ Silicon dioxide - $3.8 \%$

Calcium hydroxide - $0.95,1.4$,

Secondary Material

1.9 , and $5.7 \%$

Material Form*

Powder plus powder
Type of Soil Treated

Clayey silt
Cost

Not given 
Category*

Silicate/Other

Basic Material

Rate of Material

Cost

Sodium silicate plus Varied (see comments)

basic magne sium carbonate

Secondary Material

Material Form*

Powder plus powder
Type of Soil Treated

Silt
Mixing

Capability

Good

\begin{tabular}{|c|c|c|c|}
\hline Type of Test & $\begin{array}{c}\text { Purpose of } \\
\text { Material }\end{array}$ & $\begin{array}{l}\text { Effective } \\
\text { Strength } \\
\text { Increase } \\
\end{array}$ & Effectiveness \\
\hline $\begin{array}{l}\text { Unconfined } \\
\text { compression }\end{array}$ & Stabilizer & See comments & Excellent \\
\hline
\end{tabular}

Total Material Cost

Per Cu Ft

of Treated Soil

Not given

\section{Test Agency}

MIT
Test Report

Reference 40

\section{Comments:}

Samples treated with basic materials were not compared to untreated samples. (Sodium silicate is a combination of silicon dioxide and sodium oxide.) The effects of varying the silica, magnesium, and sodium contents were studied. For each test, two of the components were held at the same rate while the rate of the third one varied.

(Continued on next page)

* Basic material 
Effectiveness: Silica content varied. 2.51, 3.82, and 5.12 percent with magnesium (1. 8 percent) and sodium (1.59 percent) constant. Highest strength achieved was 180 psi at 5.12 percent silica.

Magnesium content varied. 1.20, 1.80, and 2. 40 percent with silica ( 5.12 percent) and sodium (1.59 percent) const ant. Highest strength achieved was 235 psi at 2 : 40 percent magnesium.

Sodium content varied. $1.59,2.14$, and 3.24 percent with silica (5.12 percent) and magnesium (1.80 percent) constant. Highest strength achieved was $350 \mathrm{psi}$ at the 2.14 percent sodium.

The most effective combination for stabilization was silica (5.12 percent), magnesium (1.80 percent), and sodium (2.14 percent) 350 psi. 
Category: Other 
Category*

Other

Basic Material

Chrome lignin

$\frac{\text { Rate of Material }}{5 \%}$

Cost

Not given

Secondary Material

Material Form*

Powder
Type of Soil Treated Loess
Mixing

Capability

Good

Total Material Cost

Per Cu Ft

Type of Test

Purpose of

Effective

Unconfined

compression

Stabilizer

Waterproofer

Effectiveness

$335 \%$

Excellent
Test Agency

WES
Test Report

Reference 24

\section{Comments:}

Treated samples were compared to untreated samples ( 23 psi unconfined compression strength). Samples prior to tests were air-dried for 4 days followed by 4 days wetting by permeation. The strength of the treated samples was 100 psi which was an increase of 335 percent. The material showed promise as a waterproofer.

This material was also subjected to field investigations at WES as a dustproofer and waterproofer. However, the results did not indicate the need for further tests of this material.

* Basic material 
Category*

Other

Basic Material

$\frac{\text { Rate of Material }}{1 \%(5 \%)}$

Cost

Lignin (clarion extract)

Not given

Secondary Material

Sodium dichromate

$0.17 \%(0.82 \%)$

Not given

Sulfuric acid

$0.17 \%(0.82 \%)$

Sodium chloride

$0.17 \%(0 \%)$

Material Form*

Liquid

Type of Soil Treated

Clay

Not given

Mixing

Capability

Good

\begin{tabular}{|c|c|c|c|}
\hline Type of Test & $\begin{array}{l}\text { Purpose of } \\
\text { Material }\end{array}$ & $\begin{array}{l}\text { Effective } \\
\text { Strength } \\
\text { Increase }\end{array}$ & Effectiveness \\
\hline $\begin{array}{l}\text { Unconfined } \\
\text { compression }\end{array}$ & Stabilizer & See comments & See comments \\
\hline
\end{tabular}

Total Material Cost

Per Cu Ft

of Treated Soil

Not given

Test Agency

Test Report

Cornell University Reference 5

Comments:

Treated samples were compared to untreated samples. These samples were allowed to air cure for varying amounts of time. Comparisons of strengths are given below. The numbers in parentheses give the amount of each material used in a second test.

(Continued on next page)

* Basic material 


\begin{tabular}{|c|c|c|c|c|}
\hline $\begin{array}{l}\text { Basic } \\
\text { Material }\end{array}$ & $\begin{array}{c}\text { Basic } \\
\text { Material } \\
\% \\
\end{array}$ & $\begin{array}{c}\text { Cure Time } \\
\text { Deys } \\
\end{array}$ & $\begin{array}{c}\text { Strength } \\
\text { psi }\end{array}$ & $\begin{array}{l}\text { Strength Change } \\
\text { Based on } \\
\text { Untreated Samples } \\
\% \\
\end{array}$ \\
\hline None & 0 & 2 & 83 & -- \\
\hline None & 0 & 9 & 210 & - \\
\hline None & 0 & 28 & 407 & - \\
\hline Lignin & 1 & 1 & 25 & Negative \\
\hline Lignin & 1 & 29 & 541 & +33 \\
\hline Lignin & 5 & 2 & 71 & Negative \\
\hline Lignin & 5 & 14 & 404 & +93 \\
\hline
\end{tabular}

Effectiveness: After long periods of time, samples treated with $I$ and 5 percent lignin have an increase in strength with the 5 percent treatment the most effective. 


\section{Category* \\ Other}

Basic Material

Rate of Material

Cost

Powder A plus powder B

6.5 and $13 \%$

Not given

Secondary Material

$\frac{\text { Material Form* }}{\text { Powder }}$
Type of Soil Treated

loess and heavy clay

$\frac{\text { Type of Test }}{\text { Unconfined }}$ compression
Purpose of

$\frac{\text { Material }}{\text { Stabilizer }}$

Effective

Strength

Increase

See comments

Effectiveness

Good

Total Material Cost

Per Cu Ft

$\frac{\text { of Treated Soil }}{\text { Not given }} \quad \frac{\text { Test Agency }}{\text { WES }}$

Comments:

Treated samples were compared to untreated samples (24 psi). Samples were prepared with a Harvard miniature compaction apparatus, five layers, each layer ten tamps of a 40-lb spring tamper. Prior to tests, samples were cured at 100 percent relative humidity followed by 24 hours water immersion.

Effectiveness: Loess - the 6.5 and 13 percent rates produced strength increases of 259 and 389 percent over the untreated samples. immersion.

* Basic material 


\section{Category* \\ Other}

Basic Material

SA-1

Secondary Material
Liquid
Material Form*
Rate of Material

See comments
Cost

Not given
Mixing

Capability

\begin{tabular}{|c|c|c|c|}
\hline Type of Test & $\begin{array}{l}\text { Purpose of } \\
\text { Material }\end{array}$ & $\begin{array}{l}\text { Effective } \\
\text { Strength } \\
\text { Increase }\end{array}$ & Effectiveness \\
\hline $\begin{array}{l}\text { Unconfined } \\
\text { compression }\end{array}$ & Stabilizer & See comments & - \\
\hline
\end{tabular}

Total Material Cost

Per Cu Ft

\begin{tabular}{|c|c|c|}
\hline Not given & WES & $\begin{array}{l}\text { Internal Dat } \\
\text { (1974), not } \\
\text { published }\end{array}$ \\
\hline
\end{tabular}

Comments:

Preparation of the samples was with a Harvard miniature compaction apparatus, ten tamps on each of five layers with a 40-lb spring tamper. The treated samples were compared to untreated samples.

Rate of material: Lean clay - 0.5 milliliter SA-l to 99.5 milliliter of water

1 milliliter SA-1 to 999 milliliter water

1.5 milliliters SA-1 to 998.5 milliliters water

(Continued on next page)

* Basic material 
2 milliliters SA-1 to 998 milliliters water

Heavy clay - 0.5 milliliters SA-1 to 999.5 milliliters water 2

2 milliliters SA-I to 999 milliliters water

Effectiveness: Lean clay - The only rate that met the requirements of Category 2 stabilization was the third rate above.

Heavy clay - The only rate that met the requirements of Category 2 stabilization was the second rate above.

Although the rates stated met the requirements of Category 2 stabilization, portland cement at 6 percent gave higher rates and is a cheaper material. 


\section{Category* \\ Other}

Basic Material

Sundcrete

Secondary Material
Rate of Material $3 \%$
Cost

Not given $\frac{\text { Material Form* }}{\text { Liquid }}$
Type of Soil Treated

Lean clay and sand
Mixing

Capability

Good

\begin{tabular}{|c|c|c|c|}
\hline Type of Test & $\begin{array}{c}\text { Purpose of } \\
\text { Material }\end{array}$ & $\begin{array}{l}\text { Effective } \\
\text { Strength } \\
\text { Increase }\end{array}$ & Effectiveness \\
\hline $\begin{array}{l}\text { Unconfined } \\
\text { compression }\end{array}$ & Stabilizer & See comments & $\begin{array}{l}\text { Excellent for } \\
\text { clay }\end{array}$ \\
\hline
\end{tabular}

Total Material Cost

Per Cu Ft

of Treated Soil

Not given

$\frac{\text { Test Agency }}{\text { WES }}$

WES
Test Report

Internal Data

(1972), not published

Comments:

Preparation of samples was with a Harvard miniature compaction apparatus using ten tamps on each of five layers with a 20-lb spring tamper. Treated samples of the lean clay soil were compared to untreated samples. The untreated sand samples fell apare and could not be tested.

Effectiveness: Sand-After 24 hours humid cure, the strength of two samples was 144 and 186 psi. Two other samples were, in addition to the 24 hours humid cure, immersed in water for 24 hours. The strengths of these samples were 228 and 231 psi. Sand treated samples therefore met the requirements of Category 2 stabilization.

Lean clay - Slight increase in strength; however, not enough to satisfy Category 2 stabilization.

* Basic material 
Category*

Other

Basic Material

Sodium methylethyl

propyl siliconate

$\frac{\text { Rate of Material }}{1.0 \%}$

Cost

Not given

Secondary Material

$\frac{\text { Material Form* }}{\text { Liquid }}$ $\frac{\text { Type of Soil Treated }}{\text { Loess }}$

Effective

Streng th

Type of Test

Unconfined

compression
Purpose of Material

Stabilizer

Waterproofer
Mixing

Capability

Good
Effectiveness

Excellent

Total Material Cost

Per Cu Ft

of Treated Soil

Not given

Test Agency

WES
Test Report

Reference 24

\section{Comments:}

Treated samples were compared to untreated samples (23 psi unconfined compression strength.) Samples prior to tests were air-dried for 4 days wetting by permeation. The strength of the treated samples was 119 psi. which was an increase of 417 percent. The material showed promise as a waterproofer.

This material was also subjected to field investigations at the WES as a dustproofer and waterproofer. The results indicated that further tests of the material were warrented.

* Basic material 
Category*

Other

Basic Material

Soil-Set

Rate of Material

$3,7,10,20$, and $30 \%$

$\frac{\text { Cost }}{\$ 0.75 \text { per } 1 b}$

Secondary Material

$\frac{\text { Material Form* }}{\text { Powder }} \quad \frac{\text { Type of Soil Treated }}{\text { Lean clay, heavy clay, and }} \quad \frac{\begin{array}{c}\text { Mixing } \\ \text { sand }\end{array}}{\text { Good }}$

\begin{tabular}{|c|c|c|c|}
\hline Type of Test & $\begin{array}{c}\text { Purpose of } \\
\text { Material }\end{array}$ & $\begin{array}{l}\text { Effective } \\
\text { Strength } \\
\text { Increase }\end{array}$ & Effectiveness \\
\hline $\begin{array}{l}\text { Unconfined } \\
\text { compression }\end{array}$ & Stabilizer & See comments & $\begin{array}{l}\text { Excellent for } \\
\text { clay }\end{array}$ \\
\hline
\end{tabular}

Total Material Cost

Per Cu Ft

\begin{tabular}{|c|c|c|}
\hline $\begin{array}{l}\text { Cost will vary from } \$ 2.50 \\
\text { to } \$ 7.00 \text { per sq yd per in. }\end{array}$ & WES & $\begin{array}{l}\text { Internal Data (1966), } \\
\text { not published }\end{array}$ \\
\hline
\end{tabular}

\section{Comments:}

Treated samples were compared to untreated samples. Samples-when tested to satisfy emergency requirements were prepared in a Harvard miniature compaction apparatus, ten tamps on each of five layers with a 20-1b spring tamper. Samples were cured for 2 hours' in 100 percent relative humldity and then subjected to tests. Samples when tested to satisfy routine requirements were prepared in a Harvard miniature compaction apparatus, ten tamps on each of five layers with a 40-1b spring tamper. Tests were then conducted after a 24-hour cure of the samples under 100 percent relative humidity. Other samples were subjected to 24 hours humid cure followed by 24 hours water immersion.

(Continued on next page)

* Basic material 
Effectiveness: Emergency requirements: Approximately 14 percent and 8 percent Soil-set are required to increase the strength of the lean and heavy clay, respectively, from 1 to 2 psi to 20 psi or higher in 2 hours.

Routine requirements: Approximately 6.5 and 9.0 percent of SollSet are required for lean and heavy clay, respectively, to increase the strength from 20 to 100 psi in 24 hours.

The strength developing ability of Soil-Set treated fine sands is a function of water content. For water content of 5 to 10 percent, approximately 15 percent Soil-Set by dry soil weight is required to satisfy routine requirements. Excessively wet sands (water content $>20$ percent) do not respond to treatment by Soil-Set. 